\title{
HOMOTOPICAL DYNAMICS IV: HOPF INVARIANTS AND HAMILTONIAN FLOWS
}

\author{
OCTAVIAN CORNEA
}

\section{INTRODUCTION.}

Let $(M, \omega)$ be an $n$-dimensional, symplectic, not necessarily compact manifold. Suppose that $f$ is a $C^{2}$, real function on $M$. A fundamental question is whether the hamiltonian flow $h^{f}$ induced by $(f, \omega)$ has periodic orbits. Many results are known when $M$ or some of the level surfaces of $f$ have compact components or, at least, are of finite volume. However, if this is not the case - and our focus will be on this non-compact case - very few results exist ( see [11] [12] as well as the discussion at the beginning of $\S_{3}$ ).

In this non-compact context the first natural step in the search for periodic orbits is to detect bounded ones. The key underlying idea of this paper is that algebraic topological constraints imposed to certain invariants associated to a gradient flow of $f$ imply the existence of bounded orbits for $h^{f}$. Once the existence of bounded orbits is established, under favorable circumstances, application of the $C^{1}$-closing lemma leads to periodic ones.

Fix a riemannian metric $\alpha$ on $M$. For technical reasons, we will assume in this paper that $M$ is simply-connected. Let $S$ be an isolated, compact, invariant set of the negative gradient flow $\gamma(f)$ of $f$ [2]. For such an $S$ we consider two associated invariants. The first, $c(S)$, is the Conley index [2]. The second invariant $d(S) \in \mathbf{Z} / 2$ is new and of a different type. Here are some consequences of our results:

Assume that the negative gradient flow of the function $f: M \rightarrow R$ has an isolated invariant set $S$ such that $d(S)=1$ and $S$ satisfies a technical property $(*)$ (that will be discussed below).

(i) If $f$ is Morse, then there are infinitely many regular hypersurfaces $f^{-1}(a)$ which after deformation by an arbitrarily small, compactly supported isotopy carry a closed characteristic.

(ii) For a general $f$ there is some $C^{2}$-neighbourhood of $f$ in the Whitney (strong) topology containing a dense family of functions each of whose hamiltonian flow has infinitely many distinct, periodic orbits.

These results are non-trivial because, in general, the hypersurfaces $f^{-1}(a)$ appearing above have no compact components. Some explicit such examples

Date: 22.04 .2001 . 
of connected, non-compact hypersurfaces $V$ are given in $\S 3.3$. We shall also see there that there are $(f, S)$ such that property $(*)$ is satisfied, $h^{f}$ has no non-trivial bounded orbit and the family of hamiltonians that have some non-trivial bounded orbit is not dense in any neighbourhood of $f$ (obviously in this case $d(S)=0$ ). In fact, under the assumptions at (i) above we obtain that all hypersurfaces $f^{-1}(a)$ that appear in that statement (before deformation) contain at least one bounded orbit of $h^{f}$.

In many cases, $d(S)$ can be computed homotopically out of an index pair $\left(N_{1}, N_{0}\right)$ (in the sense of the Conley index theory, see [2] 24] and also 8 [1.1) of $S$. By definition, $c(S)$ is the homotopy type of $N_{1} / N_{0}$. As for $d(S)$, an important property is that it equals 1 if one of the following two conditions is satisfied: a. $c(S)$ has torsion in homology; b. there exists an index pair for $S$ with $N_{1}$ and $N_{0}$ both simply-connected, the homology of $c(S)$ is torsion free and a certain chain complex $\mathcal{C}_{\min }\left(N_{1}, N_{0}\right)$ of free $\pi_{*}^{S}\left((\Omega M)^{+}\right)$modules canonically associated to a minimal $C W$-cell decomposition of the pair $\left(N_{1}, N_{0}\right)$ has a non trivial differential. We call this complex, which is unique up to isomorphism and is closely related to the twisted complexes introduced by Baues [1] in homotopy theory, the minimal Hopf complex of $\left(N_{1}, N_{0}\right)$. This name is motivated by the fact that the differential is expressed in terms of certain relative Hopf invariants associated to succesive cell-attachments in a minimal cell-decomposition of $\left(N_{1}, N_{0}\right)$. There are reasonably simple criteria implying the non-vanishing of the differential, in particular one in terms of the non-vanishing of certain differentials in the Atiyah-Hirzebruch-Serre $\pi_{*}^{S}\left(\Omega M^{+}\right)$-spectral sequence of the fibration induced over $\left(N_{1}, N_{0}\right)$ from the path-loop fibration $\Omega M \rightarrow P M \rightarrow M$.

Here is now property $(*)$ : we say that $S$ satisfies the property $(*)$ (with respect to $v \in \mathbf{R})$ if for a value $v \in \mathbf{R}$ there exists an index pair $\left(N_{1}, N_{0}\right)$ for $S$ (in the flow $\gamma(f))$ such that $N_{0} \subset f^{-1}(v)$ and $f$ is regular on $N_{0}$. There are many simple criteria that insure that this condition is satisfied (see Lemma 3.7).

We explain below the ideas used in the proof of the results mentioned above and, at the same time, describe the contents of the paper.

Given a gradient flow of $f$ and an isolated invariant set $S$ in this flow, Conley's theory provides a way to associate some homotopy theoretical meaningful notions to $(f, S)$. In particular, because we are talking here about a gradient flow on a finite dimensional manifold, isolating neighbourhoods as well as index pairs of $S$ are generally not difficult to determine. Assuming such an index pair $\left(N_{1}, N_{0}\right)$ constructed the main aim becomes to translate the homotopical properties of this pair into recurrence properties of $h^{f}$.

Notice that even if the function $f$ is not Morse itself it can be morsified in a neighbourhood of $S$. In other words, given an isolating neighbourhood $N$ of $S$ we may continue $S$ inside $N$ to an isolated invariant set $S^{\prime}$ of the gradient flow of a function $g$ which agrees with $f$ outside $N$ and satisfies the Morse-Smale condition inside $N$. Such a $g$ can be constructed to be as close 
to $f$ in the $C^{2}$-strong topology as desired. Consider couples $(g, N)$ as above. Explicitely, $N$ is an isolating neighbourhood of the negative gradient flow of $g$ and, inside $N, g$ is Morse and satisfies the Morse-Smale transversality condition. To such a couple - which will be called a local Morse function - we associate certain invariants which make the connection between the homotopy type of the pair $\left(N_{1}, N_{0}\right)$ and the existence of bounded orbits of $h^{g}$.

These invariants are Morse-type complexes only that, instead of measuring the space of flow lines joining critical points (of $\left.g\right|_{N}$ ) by just counting the number of its elements (when this number is finite), we consider consecutive critical points of indexes different by possibly more than one but successive in a fixed index set $I \subset \mathbf{N}$ and measure the corresponding space of connecting flow lines (called connecting manifold) by a certain framed bordims class that belongs to $\Omega_{*}^{f r}(\Omega M)$ (the precise definition is in $\left.\$ 1.2\right)$. We denote the resulting $\Omega_{*}^{f r}(\Omega M)$-chain complexes by $C_{N}^{I}(g)$ and we call them extended Morse complexes of $g$ (relative to $N$ ). The invariant $d(S)$ is defined in terms of these complexes (see $\S 3.3): d(S)=1$ if there is a $C^{2}$-neighbourhood, $\mathcal{U}_{f}$, of $f$ such that for each morsification $(g, N)$ of $S$ with $g \in \mathcal{U}_{f}$ there is an extended Morse complex $C_{N}^{I}(g)$ with non-trivial differential.

The paper is structured by the point of view that extended Morse complexes, introduced in Section 1, provide a bridge between homotopy theory and recurrence properties of the respective hamiltonian flows. The two other sections of the paper deal each with one of the two sides of the story.

The relation between extended Morse complexes and homotopy theory is the task of Section 2. Assume $(g, N)$ is a local Morse function and let $P$ and $Q$ be consecutive critical points of $\left.g\right|_{N}$ (which means $p=i n d(P)>$ $\operatorname{ind}(Q)=q$ and $N$ does not contain broken flow lines of the gradient flow of $g$ joining $P$ to $Q$ ). The geometry of the connecting manifold of $P$ and $Q$ produces, by the Thom-Pontryaguin construction, (see again $\delta[1.2$ ) a homotopy class $\left.h(P, Q) \in \pi_{p-1}\left(\Sigma^{q}(\Omega M)^{+}\right)\right)$whose stabilization is the bordism class mentioned above. The main result of this section shows that $h(P, Q)$ equals a certain homotopical object called the relative Hopf invariant associated to the cell attachments corresponding to the "passage" through $P$ and $Q$ (this extends results in 10] and [5]). In particular, this allows the identification of the extended Morse complexes of $(g, N)$ with Hopf - type complexes associated to a relative, not necessarily minimal, cell-decomposition of the index pair $\left(N_{1}, N_{0}\right)$ (recall that $\Omega_{*}^{f r}(A) \approx \pi_{*}^{S}\left(A^{+}\right) ; A^{+}=(A)^{+}=A$ union a disjoint point). As a corollary of this identification we prove in $\$ 2.3$ some rigidity properties of these extended Morse complexes. In particular, returning to the function $f$ and the isolated invariant set $S$, if the index pair $\left(N_{1}, N_{0}\right)$ has torsion free integral relative homology and both $N_{1}$ and $N_{0}$ are simply connected, then $\mathcal{C}_{\text {min }}\left(N_{1}, N_{0}\right)$ is defined and, if the differential in 
$\mathcal{C}_{\min }\left(N_{1}, N_{0}\right)$ is non-trivial, then the differential of the complex $C_{N}^{I_{g}}(g)$ does not vanish either for any morsification $(g, N)$ of $S$ and some index set $I_{g}$. It also follows that if $c(S)$ has torsion in homology, then the differential of such a complex $C_{N}^{I_{g}}(g)$ is again non-trivial. This leads to the fact that in both these two cases $d(S)=1$.

In Section 3 we relate the differential of extended Morse complexes to hamiltonian flows. It is only here that property $(*)$ is of use. For a local Morse function $(g, N)$ whose maximal invariant set $S^{\prime}$ inside $N$ satisfies condition $(*)$ the main result of this section shows that the non-vanishing of the differential in $C_{N}^{I_{g}}(g)$ implies that the hamiltonian flow of $g$ has some bounded orbits situated on regular hypersurfaces. The (very) rough idea of the proof is as follows. The non-vanishing of the differential above implies that some bordism class $[h(P, Q)]$ is non-trivial. Let $V=g^{-1}(a)$ be such that it separates $P$ and $Q, g(P)>g(Q)$. We show that if the flow $h^{g}$ has no bounded orbits on $V$, then the connection between $P$ and $Q$ given by the negative gradient flow lines of $g$ can be annihilated by letting the unstable sphere of $P$ in $V, W^{u}(P) \cap V$, be deformed by (a perturbation of) $h^{g}$ till it becomes disjoint from the stable sphere of $Q$ in $V, W^{s}(Q) \cap V$. This leads to a contradiction because it would imply that the bordism class $[h(P, Q)]$ is trivial. It is useful to note that this type of argument actually does apply to any flow orthogonal to the gradient flow of $g$. We use the $C^{1}$ closing lemma of Pugh and Robinson [21] to deduce the existence of periodic orbits for the flow $h^{g^{\prime}}$ for some functions $g^{\prime}$ close to $g$ as well as to obtain the existence of closed characteristics on some hypersurfaces close to those of $g$. This immediately leads to a proof of the points (i) and (ii) above. When $d(S)=0$ or the condition $(*)$ is not satisfied a different method is available. This continues an idea that first appeared in [6]. It makes use of the dynamical interpretation of Spanier-Whitehead duality first discussed in [4] to show that, under certain circumstances, the lack of self-duality of the Conley index of a compact invariant set in a gradient flow can force the appearance of some non-constant, bounded orbits for the associated hamiltonian flow. A result obtained in this way claims the existence of a family of functions dense in a neighbourhood of $f$ each of whose induced hamiltonian flow has at least as many closed (possibly homoclinic), distinct, non-trivial orbits as $\sum_{k \neq n / 2} r k\left(H_{k}(c(S) ; \mathbf{Z})\right.$.

Section 2 applies to local Morse functions on a manifold $M$ that is not necessarily symplectic. The results contained here are of interest independentely of their applications in Section 3. Indeed, at the center of this series of papers is the old idea, going back to the work of Poincaré, that homotopy theory is relevant in understanding flows. The first systematic attempt to go beyond applications of homology in this direction have been provided by Conley's theory [2] and by work of John Franks, particularly in [10]. A 
key point is that, essentially, Conley's approach only provides information about the simplest existing flow inside an isolating neighbourhood if the boundary behaviour is fixed. This means that it is not only natural but also quite meaningful to first try to understand the simplest isolated invariants sets possible - isolated invariant points in gradient flows - and the simplest attractor-repellor pairs possible - which are consecutive critical points for Morse-Smale functions together with the gradient flow lines that connect them. Isolated singularities of real functions have been studied from this perspective in [6]. In [10] John Franks has started the application of more refined homotopical tools to the study of these "simplest" attractor-repellor pairs formed by consecutive critical points in Morse-Smale gradient flows. His results have been extended by the author in [5] and this work is continued and strengthened here. Extended Morse complexes provide a tool to encode the data coming from these attractor-repellor pairs in an algebraic way and, therefore, their study seems to be a worthwhile topic in itself. Because of this we include in Section 2 more properties of these complexes than those strictly needed later in the paper. In particular, we discuss a connection between this topic and the problem of the existence of smoothings of Poincaré duality spaces (along the lines of [5]).

Most of the paper is written in the language of Conley's index theory. A notable exception is $\$ 2.1$ which concentrates all the non-trivial homotopy theoretical arguments of the paper. This sub-section is of some intrinseque interest and can be read independently of the rest of the paper.

To make the paper as widely accessible as possible all the needed elements of Conley index theory together with the basics of Morse theory that we use are recalled in Section 11.

\section{CONTEnTs}

Introduction.

1. Recalls and preliminary definitions. 6

$\begin{array}{ll}\text { 1.1. The Conley index. } & 6\end{array}$

1.2. $\quad$ Elements of Morse theory and extended Morse complexes. 8

1.3. Cofibrations and relative Hopf invariants. 11

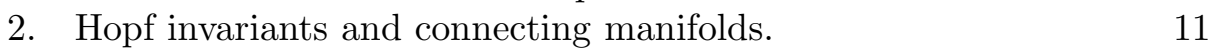

2.1. Hopf invariants as relative attaching maps. 13

2.2. Relating Hopf invariants and bordism maps. 23

2.3. Rigidity and duality for extended Morse complexes. 28

3. Bounded, closed and periodic orbits for hamiltonian flows. $\quad 33$

3.1. Lack of self - duality and closed orbits. 34

3.2. Non-vanishing of Hopf invariants and periodic orbits. $\quad 37$

3.3. Applications and examples. 45

$\begin{array}{ll}\text { References } & 53\end{array}$ 


\section{ReCAlls And PRELIMinary DEFinitions.}

This section does not contain new results or proofs. It serves two purposes. The first is to fix notations and standard facts concerning Morse functions and the Conley index. The second is to introduce the definitions of "generalized" and "extended" Morse complexes.

\subsection{The Conley index.}

We recall some elements of Conley index theory [2] [24].

Let $\gamma: X \times \mathbf{R} \longrightarrow X$ be a continuous flow on a locally compact, metrizable space $X$. Given a subset $N \subset X$ we denote by $I_{\gamma}(N)$ or $I(N)$ the maximal invariant set of $\gamma$ included in $N$. A compact $N \subset X$ is called isolating neighbourhood in the flow $\gamma$ if $I(N) \subset \operatorname{Int}(N)$. It follows that $I(N)$ is compact. In other words a compact $N \subset X$ is an isolating neighbourhood if all orbits of type $\gamma_{\mathbf{R}}(x), x \in N$ that are included in $N$ do not intersect $\partial N$. An invariant set of $\gamma, S \subset X$ is isolated if $S=I(N)$ for some isolating neighbourhood $N$. Fix now an isolated invariant set $S$ inside some isolating neighbourhood $N$ in the flow $\gamma$. A pair $\left(N_{1}, N_{0}\right)$ of compact subsets of $N$ is an index pair for $S$ in $N$ if $N_{0} \subset N_{1}, N_{1}-N_{0}$ is a neighbourhood of $S$, $S=I\left(\overline{\operatorname{Int}\left(N_{1}-N_{0}\right)}\right), N_{0}$ is positively invariant in $N_{1}$ and, if for $x \in N_{1}$ there is some $T \geq 0$ such that $\gamma_{T}(x) \notin N_{1}$, then there exists a $\tau>0, \tau<T$ with $\gamma_{t}(x) \in N_{1}$ for $0 \leq t \leq \tau$ and $\gamma_{\tau}(x) \in N_{0}$. For such an index pair $\left(N_{1}, N_{0}\right)$ we will call $N_{0}$ the exit set. A key basic result is that there are index pairs inside any isolating neighborhood of $S$. The Conley index of $S$, $c_{\gamma}(S)$, is the homotopy type of the quotient space $N_{1} / N_{0}$. It is independent of the choices of the index pair. It is also invariant to continuation in the sense that if $\gamma^{\lambda}: X \times \mathbf{R} \longrightarrow X$ is a family of flows depending continuously on the parameter $\lambda \in[0,1]$ and $S$ is an isolated invariant set of $\gamma$ viewed as a flow on $X \times[0,1]$, then $S_{\lambda}=S \bigcap X \times\{\lambda\}$ is an isolated invariant set of $\gamma^{\lambda}$ and $c_{\gamma}(S)=c_{\gamma^{\lambda}}\left(S_{\lambda}\right)$ for all $\lambda \in[0,1]$. For an arbitrary point $x \in X$ let its $\omega$ and $\omega^{*}$ limits be defined by

$$
\omega(x)=\left\{z \in X: \exists\left\{t_{n}\right\} \rightarrow+\infty, z=\lim _{n \rightarrow \infty} \gamma_{t_{n}}(x)\right\}
$$

and

$$
\omega^{*}(x)=\left\{z \in X: \exists\left\{t_{n}\right\} \rightarrow-\infty, z=\lim _{n \rightarrow \infty} \gamma_{t_{n}}(x)\right\}
$$

If $N$ is an isolating neighbourhood of the isolated invariant set $S$, then we denote by

$$
W_{N}^{u}(S)=\left\{x \in N: \forall t \in(-\infty, 0], \gamma_{t}(x) \in N, \omega^{*}(x) \subset S\right\}
$$

and

$$
W_{N}^{s}(S)=\left\{x \in N: \forall t \in[0, \infty), \gamma_{t}(x) \in N, \omega(x) \subset S\right\}
$$

the unstable and stable manifolds of $S$ in $N$. Both sets are compact. We will generally omit the index $N$ from the notation if this does not create any ambiguity. A special type of index pairs that are particularly useful are regular index pairs [24]. Two of their important properties are that $N_{0}$ is 
a neighbourhood deformation retract and that the arrival time in $N_{0}$ is a continuous function on $N_{1}-W_{N_{1}}^{s}(S)$ where $S$ is the maximal invariant set inside $N_{1}$. We also need the notion of bi-regular index block. This is a triple $\left(N_{1}, N_{0}, N_{2}\right)$ such that $\left(N_{1}, N_{0}\right)$ and $\left(N_{1}, N_{2}\right)$ are respectively regular index pairs for the direct and inverse flows and $N_{0} \cup N_{2}$ includes $\partial N_{1}$ (see [23]; the inverse flow of $\gamma$ is defined by $-\gamma_{t}(x)=\gamma_{-t}(x)$ ). In our case, $\gamma$ will always be defined on a smooth manifold and is differentiable of class at least $C^{1}$. We will also need a special type of bi-regular index block that we call strong index block. This is a triple $\left(N_{1}, N_{0}, N_{2}\right)$ such that $N_{1}$ is a submanifold of the same dimension as the underlying manifold, it has a smooth interior, $\partial N_{1}=N_{0} \cup N_{2}$ with $N_{0}$ and $N_{2}$ smooth manifolds possibly with boundary $\partial N_{0}=\partial N_{2}=N_{0} \cap N_{2}$ and such that the vector field $X(\gamma)$ associated to $\gamma$ is never tangent to $\partial N_{1}$ and points strictly inside $N_{1}$ on $N_{2}$ and strictly outside on $N_{0}$. Such a strong index block can be easily constructed inside any isolating neighbourhood by making use of Lyapounov functions for the direct and inverse flows 24]. Here is a simple but important property of strong index blocks. Fix $\left(N_{1}, N_{0}, N_{2}\right)$ a strong index block for $\gamma$. If $\gamma^{\prime}$ is a flow such that $X\left(\gamma^{\prime}\right)$ is sufficientely $C^{0}$-close to $X(\gamma)$ on $\partial N_{1}$, then $\left(N_{1}, N_{0}, N_{2}\right)$ is also a strong index block for $\gamma^{\prime}$. By using the one parameter family of flows induced $t X(\gamma)+(1-t) X\left(\gamma^{\prime}\right)$, it results that the maximal invariant sets of $\gamma$ and $\gamma^{\prime}$ inside $N_{1}$ are related by continuation. This construction is useful in continuing a given isolated invariant set $S$ of some flow $\gamma$ to simpler invariant sets. In particular, it is easy to show by using the two Lyapounov functions mentioned above that, on a smooth manifold, any isolated invariant set can be continued to one in a gradient flow.

The Conley index also associates some relevant homotopy data to an attractor-repellor pair $(S ; R, A)$ in the flow $\gamma$. This is an isolated invariant set such that $A, R \subset S$ are disjoint isolated invariant sets and any $x \in$ $S-(A \cup R)$ has an verifies $\omega(x) \subset A, \omega^{*}(x) \subset R$. To such an attractor repellor pair there coresponds a homotopy cofibration sequence [24] called the attractor-repellor cofibration sequence:

$$
c_{\gamma}(A) \rightarrow c_{\gamma}(S) \rightarrow c_{\gamma}(R) \stackrel{\delta(R, A)}{\rightarrow} \Sigma c_{\gamma}(A)
$$

One important feature is that the map $\delta(R, A)$ admits a flow based explicit defintion besides the one coming from the Baratt-Puppe sequence. This map is called the connection map of the attractor repellor pair.

There is a variant of the Conley index that has been recentely introduced by Mrozek, Reineck and Srzednicki [18] and which is useful in our context. For a flow $\gamma$ on $X$ having an isolated invariant set $S$ consider a regular index pair $\left(N_{1}, N_{0}\right)$ of $S$. Consider the space $X^{\prime}$ obtained from the disjoint union of $X$ and of $N_{1}$ modulo the identification of $N_{0} \hookrightarrow N_{1}$ with $N_{0} \hookrightarrow X$. Of course $X \subset X^{\prime}$ and there is also a map $p: X^{\prime} \rightarrow X$ which is the identity on $X$ and the inclusion on $N_{1}$. The global Conley index of $\gamma$ as defined in [18] is denoted by $\bar{c}_{\gamma}(S)$ and it is the homotopy type (in the obvious sense) of the object given by the triple $\left(X^{\prime}, X, p\right)$. The main result in 18$]$ is that this 
global Conley index satisfies all the properties of the usual one, in particular it is independent of the choice of index pairs and is invariant to continuation. All algebraic topological invariants (i.e. homology or homotopy groups) of $\bar{c}_{\gamma}(S)$ will be understood to be the respective invariants of the pair $\left(X^{\prime}, X\right)$.

The notions above adapt in an obvious way to local flows 24]. This is particularly useful because many of the flows that we consider in this paper are in fact only partially defined (due to the non-compactness of our underlying manifold) but they are all local flows.

\subsection{Elements of Morse theory and extended Morse complexes.}

Let $M$ be our fixed, smooth, connected, not necessarily compact, $n$ dimensional manifold with a fixed riemannian metric $\alpha$. For a $C^{1}$ function $f: M \rightarrow \mathbf{R}$ we denote by $\nabla(f)$ the $\alpha$-gradient vector field of $f$ and let $\gamma(f)$ be the (partially defined) flow induced by $-\nabla(f)$.

Definition 1. A local Morse function on $M$ is a pair $(f, N)$ such that $f$ : $M \rightarrow \mathbf{R}$ is smooth, $N$ is an isolating neighbourhood in the flow $\gamma(f),\left.f\right|_{N}$ is Morse (meaning that all critical points of $f$ inside $N$ have a non-degenerate hessian) and $f$ verifies the Morse-Smale transversality condition in $N$.

Here is the meaning of the last assumption. Let $\operatorname{Crit}^{N}(f)$ be the critical points of $\left.f\right|_{N}$ and let $C r i t_{k}^{N}(f)$ be the set of those critical points $x$ of index $\operatorname{ind}(x)=k$. For $x \in \operatorname{Crit}_{k}^{N}(f)$ the stable and unstable manifolds of $x$ in $N W_{N}^{s}(x)$ and $W_{N}^{u}(x)$ are both defined with respect to the flow $\gamma(f)$ as in $\S 1.1$. Their intersections with the interior of $N$ are, respectively, open $n-k$ and $k$-dimensional manifolds. If $N$ has the property that there are $N_{0}$, $N_{2}$ such that $\left(N, N_{0}, N_{2}\right)$ is a strong index block, then these manifolds are diffeomorphic to open euclidean disks of the corresponding dimensions. The Morse-Smale condition requires that for any $x, y \in \operatorname{Crit}^{N}(f)$ the intersection of $W_{N}^{u}(x)$ and $W_{N}^{s}(y)$ be transverse.

It is useful to notice that if $f: M \rightarrow \mathbf{R}$ is $C^{1}$ and $N$ is an isolating neighbourhood for $\gamma(f)$, then the invariant set $I_{N}(f)=I_{\gamma(f)}(N)$ is formed by all the critical points in $\operatorname{Crit}^{N}(f)$ and all the points situated on some flow line of $\gamma(f)$ that is contained in $N$ and joins two of these critical points.

In case $(f, N)$ is a local Morse function the intersection of $W_{N}^{u}(x) \cap$ $W_{N}^{s}(y) \cap f^{-1}(a), x, y \in \operatorname{Crit}^{N}(f), a \in \mathbf{R}$ regular value of $f$, is void or a compact stratified manifold with the top dimensional stratum of dimension $\operatorname{ind}(x)-i n d(y)-1$. Two critical points $P, Q \in \operatorname{Crit}^{N}(f)$ are called consecutive (in $N)$ if $f(P)>f(Q)$ and if $\overline{W_{N}^{u}(P)} \cap \overline{W_{N}^{s}(Q)} \cap C \operatorname{rit}^{N}(f)-\{P, Q\}=\emptyset$. In this case the intersection $Z_{N}(P, Q)=W_{N}^{u}(x) \cap W_{N}^{s}(y) \cap f^{-1}(a)$ is a compact manifold of dimension $\operatorname{ind}(P)-\operatorname{ind}(Q)-1$ and its diffeomorphism type does not depend of $a$. We will use the notation $Z(P, Q)$ for this manifold if $N$ is fixed.

Extended Morse complexes are a convenient way to encode some of the characteristics of the connecting maniflods of consecutive critical points. A very simple, general definition is the following. 
Definition 2. Given some graded ring $\mathcal{R}_{*}$, a generalized Morse complex associated to a local Morse function $(f, N)$ is a chain complex of free $\mathcal{R}_{*}$ modules $(\mathcal{C}, d)$ such that $\mathcal{C}=\mathcal{R}_{*} \otimes \mathbf{Z}\left[\right.$ Crit $\left._{\odot}^{N}(f)\right]$ (where $Z\left[A_{\odot}\right]$ is the graded, free abelian group generated by $\left.A_{\odot}\right)$ and for $x \in \operatorname{Crit}_{i}^{N}(f)$,

$$
d x=\sum_{y \in C r i t_{<i}^{N}(f)} d(x, y) y
$$

$d(x, y)=0$ if $x$ and $y$ are not consecutive.

The definition above becomes interesting whenever the coefficients $d(x, y)$ reflect part of the geometry of the flow $\gamma(f)$. Typically, these coefficients "measure" the connecting manifolds $Z(x, y)$. At the same time, richer the ring $\mathcal{R}$ is, finer is the structure encoded in the complex.

The classical example of a Morse complex due to Thom, Milnor, Smale appears when $\mathcal{R}=\mathbf{Z}$ and $d(x, y)$ is null except when $\operatorname{ind}_{f}(x)=i n d_{f}(y)+1$ and in that case $d(x, y)$ is equal to the number \# $(x, y)$ (or $\left.\#_{f}(x, y)\right)$ of points in $Z(x, y)$ counted with appropriate signs. We will denote this complex by $C_{N}^{M o}(f)$. Another related example that has received significant attention recentely is the Morse-Novikov complex [19].

The key example that interests us here is an extension of the classical Morse case. Consider the local Morse function $(f, N)$ and let $P \in$ $\operatorname{Crit}^{N}(f)$. Let $S^{u}(P)$ (respectively $S^{s}(P)$ ) be the unstable sphere (resp. stable sphere) of $P$ which is defined by $S^{u}(P)=W_{N}^{u}(P) \cap f^{-1}(a)$ (resp. $\left.S^{s}(P)=W_{N}^{s}(P) \bigcap f^{-1}(a)\right)$ for $a$ in between $f(P)$ and $f(P)+\epsilon$ with $\epsilon$ small in module and negative (respectively positive). The Morse lemma shows that if $\epsilon$ is sufficientely small, then $S^{u}(P)$ and $S^{s}(P)$ are both diffeomorphic to spheres of dimensions respectively ind $(P)-1$ and $n-i n d(P)-1$. Assume now that $Q \in \operatorname{Crit}^{N}(f)$ such that $P, Q$ are consecutive. By transporting $Z(P, Q)$ along the flow $-\gamma(f)$ we see that $Z(P, Q)$ is embedded in $S^{u}(P)$. It is well known [10] that $Z(P, Q)$ is actually framed in $S^{u}(P)$ with a standard normal framing. There is also a map

$$
j(P, Q): Z(P, Q) \rightarrow \Omega M
$$

defined as follows (here, and in the rest of the paper, $\Omega M$ is the space of pointed loops on $M)$. First fix a path $w$ in $M$ joining $Q$ to $P$. The map $j(P, Q)$ associates to each point $z \in Z(P, Q)$ the loop obtained by following from $P$ to $Q$ the flow line of $\gamma(f)$ that passes through $z$ and returning to $P$ via $w$. The homotopy type of this map is well defined and independent of the choice of $w$ up to conjugation by elements in $\pi_{1}(M)$. To avoid this ambiguity as well as further technical complications, we assume in this paper, as mentioned in the introduction, that $M$ is simply-connected. In this case the framed bordism classes $[Z(P, Q)] \in \Omega_{p-q-1}^{f r}(\Omega M)$ of the connecting manifolds $Z(P, Q)$ are well defined. By convention, we will put $[Z(x, y)]=0$ whenever $x$ and $y$ are not consecutive critical points. 
Definition 3. For a local Morse function $(f, N)$ let $I=\left(a_{1}, \ldots a_{i} \ldots\right) \subset \mathbf{N}$ be a strictly increasing sequence such that if $\operatorname{Crit}_{k}^{N}(f) \neq \emptyset$, then $k \in I$. The extended Morse complex of $(f, N)$ relative to $I$ is a generalized Morse complex $\left(C_{N}^{I}(f), d_{f}^{I}\right)$ with $\mathcal{R}_{*}=\Omega_{*}^{f r}(\Omega M)$ and $d_{f}^{I}(x, y)=[Z(x, y)]$ if for some $i, \operatorname{ind}_{f}(y)=a_{i}, \operatorname{ind}_{f}(x)=a_{i+1}$ and $d_{f}^{I}(x, y)=0$ otherwise.

To understand this object, the key point is to relate $d_{f}^{I}$ to homotopy theory. This is the purpose of the main theorem in I5] (in the case when $N=M$ which corresponds to the standard case of a Morse-Smale function on a compact manifold) and the results obtained there will be strengthened considerably here in Section 2 where we also show $\left(d_{f}^{I}\right)^{2}=0$.

Remark 1.1. a. Notice that not all bordism classes of connecting manifolds of consecutive critical points are contained in a Morse complex of type $\left(C_{N}^{I}(f), d_{f}^{I}\right)$ even if the index set $I$ is allowed to vary. This happens because two critical points $P$ and $Q$ might be consecutive with respect to the flow $\gamma(f)$ even if there might be a third critical point $R \in N$ of $f$ such that $\operatorname{ind}(Q)<\operatorname{ind}(R)<\operatorname{ind}(P)$.

b. The index sets $I$ above are useful in the definition of extended Morse complexes because the complexes associated to two different functions that are related by continuation and constructed with respect to the same index set $I$ will be shown to be comparable by a chain map in $\delta 2.3$.

c. There is an obvious ring map $r: \Omega_{*}^{f r}(\Omega M)=\pi_{*}^{S}\left((\Omega M)^{+}\right) \rightarrow \mathbf{Z}$. It is clear that by changing the coefficients of the complex $C_{N}^{\mathbf{N}}(f)$ by means of $r$ one obtains the classical Morse complex of $f$.

Assume $(f, N)$ is a local Morse function and fix an index pair $\left(N_{1}, N_{0}\right)$ for $I_{N}(f)$. As in classical Morse theory [15], $N_{1}$ is obtained from $N_{0}$ by the succesive attachement of precisely one $k$-handle for each critical point of $\left.f\right|_{N}$ of index $k$. Of course, the Conley index of $I_{N}(f), c_{\gamma(f)}\left(I_{N}(f)\right)$, which will be further denoted by $c_{N}(f)$, also admits a cell decomposition with one cell of dimension $k$ for each point in $\operatorname{Crit}_{k}^{N}(f)$. Moreover, the integral homology of the standard Morse complex of $f$ verifies $H_{*}\left(C^{M o}(f)\right) \approx \bar{H}_{*}\left(c_{N}(f) ; \mathbf{Z}\right)$. For further use, fix also the notation $\bar{c}_{N}(f)$ for the global Conley index of $I_{N}(f)$.

If $P, Q$ are consecutive critical points of $f$ in $N$ denote by $I(P, Q)$ the isolated invariant set consisting of the union of the points $P, Q$ and all the points situated on flow lines joining $P$ to $Q$ and contained in $N$. The triple $(I(P, Q) ; P, Q)$ is an attractor repellor pair. There is a triple $\left(N^{\prime \prime \prime}, N^{\prime \prime}, N^{\prime}\right)$ of compact subsets in $N$ such that $\left(N^{\prime \prime \prime}, N^{\prime}\right)$ is an index pair for $I(P, Q)$, $\left(N^{\prime \prime \prime}, N^{\prime \prime}\right)$ is an index pair of $P$ and $\left(N^{\prime \prime}, N^{\prime}\right)$ is an index pair for $Q$. Moreover, if $p=\operatorname{ind}(P), q=\operatorname{ind}(Q)$ there are cofibration sequences $S^{q-1} \rightarrow$ $N^{\prime} \rightarrow N^{\prime \prime}$ and $S^{p-1} \rightarrow N^{\prime \prime} \rightarrow N^{\prime \prime \prime}$. By extending the first cofibration sequence one step to the right and composing with the attaching map of $S^{p-1}$ one obtains a map $\delta: S^{p-1} \rightarrow N^{\prime} \rightarrow S^{q}$ called the relative attaching map of $P$ and $Q$. It is easy to see that we have $\delta(P, Q)=\Sigma \delta$ where $\delta(P, Q)$ is the 
connection map coming out of the attractor-repellor cofibration sequence associated to $(I(P, Q) ; P, Q)$. It was shown by Franks [10] that $\delta$ coincides up to sign with the Thom map constructed from the framed embedding $Z(P, Q) \subset W_{N}^{u}(P) \cap f^{-1}(a) \approx S^{p-1}$.

\subsection{Cofibrations and relative Hopf invariants.}

This sub-section is a digression into some elementary homotopy theory. We will work here in the pointed category of spaces with the homotopy type of $C W$-complexes. We will assume some familiarity with the language of (homotopy) fibrations, cofibrations, push-outs and pull-backs as found for example in [14.

In particular, a sequence of two maps $A \stackrel{i}{\rightarrow} X \stackrel{i^{\prime}}{\rightarrow} Y$ is a cofibration sequence if $Y=C A \cup_{i} X$ where $C A$ is the reduced cone on $A$ and the second map is the inclusion $X \hookrightarrow C A \cup_{i} X$. Given such a cofibration sequence, there is a co-action map $\nabla: Y \rightarrow \Sigma A \vee Y$ defined as the projection of $Y=C A \bigcup_{A} X$ onto $Y /(A \times\{1 / 2\})$ where $A \times\{1 / 2\} \subset C A=(A \times[0,1]) /(A \times$ $\{1\} \bigcup * \times[0,1])$. Assume that there is a second cofibration sequence $\Sigma B \stackrel{j}{\rightarrow}$

$Y \stackrel{v}{\rightarrow} Z$. We first recall that the composition $\Sigma B \stackrel{j}{\rightarrow} Y \stackrel{\nabla}{\rightarrow} \Sigma A \vee Y \rightarrow \Sigma A$ where the last map is the projection on the first term is called the relative attaching map of $\Sigma B$ and $A$ (it generalizes the map $\delta$ introduced at the end of the last paragraph).

Consider the projection on the second term $p: \Sigma A \vee Z \rightarrow Z$. The homotopy fibre of this map is homotopy equivalent to $\Sigma A \wedge\left(\Omega Z^{+}\right)$where $-^{+}$ indicates the disjoint union with a point (the precise homotopy equivalence that is used follows from diagram (8) below). It is easy to see that $\Omega k$ admits a homotopical retraction. This implies, by adjunction, that the inclusion of this fibre $k: \Sigma A \wedge\left(\Omega Z^{+}\right) \rightarrow \Sigma A \vee Z$ has the following property: if two maps out of a suspension and with values in the domain of $k$ are homotopic after composition with $k$, then the two maps are homotopic.

Let $u$ be the composition $u: \Sigma B \stackrel{j}{\rightarrow} Y \stackrel{\nabla}{\rightarrow} \Sigma A \vee Y \stackrel{i d \vee v}{\rightarrow} \Sigma A \vee Z$. Then $p \circ u$ is null-homotopic and, in view of what was said above, it has a lift $u^{\prime}: \Sigma B \rightarrow \Sigma A \wedge\left(\Omega Z^{+}\right)$that is unique up to homotopy.

Definition 4. In the setting above, the Hopf invariant of $j$ relative to $i$, $H(j, i)$, is the homotopy class of $u^{\prime}$ in $\left[\Sigma B, \Sigma A \wedge\left(\Omega Z^{+}\right)\right]$

This version of relative Hopf invariants is slightly different from that used in [5]. The relation between the two is that the projection of $H(j, i)$ on the factor $\Sigma A \wedge \Omega Z$ of $\Sigma A \wedge \Omega X^{+}=\Sigma A \vee \Sigma A \wedge \Omega Z$ equals the Hopf invariant from [5].

I am indebted to Bill Richter who has first suggested that the definition above can be of use in this paper.

\section{Hopf INVARIANTS AND CONNECTING MANIFOLDS.}


The simplest type of attractor-repellor pair is that formed by two nondegenerate consecutive critical points $P, Q$ in a Morse-Smale gradient flow together with the flow lines joining them. In this case the attractor and repellor are completely understood and the only question is to "estimate" the connecting manifold $Z(P, Q)$. Part of the answer is contained in the result of Franks [10] which provides an estimate of the framed cobordism Thom map of $Z(P, Q)$ (see above $\$ 1.2$ ). The presence of Hopf invariants in this context is natural if one views them as a homotopical way to measure the "strength" of the binding of two cells (or cones) that are attached successively.

The main result of this section, extends the result of Franks by giving a homotopical description of the Thom bordism map provided by the framing $Z(P, Q) \hookrightarrow S^{u}(P)$ and the map $j(P, Q): Z(P, Q) \rightarrow \Omega M$. More precisely, consider a fixed local Morse function $(f, N)$ (see Definition 1) Assume that $P$ and $Q$ are consecutive critical points of $f$ in $N$ of indexes respectively $p$ and $q>0$ and recall that $Z(P, Q)$ is the connecting manifold of $P$ and $Q$. As explained in $\$ 1.2, Z(P, Q)$ is a manifold of dimension $p-q-1$ which is embedded with a fixed framing in $S^{u}(P) \approx S^{p-1}$; there is also the map $j(P, Q): Z(P, Q) \rightarrow \Omega M$. The Thom-Pontryaguin construction applied to this data produces a homotopy class $h(P, Q) \in \pi_{p-1}\left(\Sigma^{q}\left(\Omega X^{+}\right)\right)$ such that the stable class of $h(P, Q)=[Z(P, Q)]$. Let $\left(N_{1}, N_{0}\right)$ be an index pair of the maximal invariant set of the negative gradient flow of $f$ inside $N, I_{N}(f)$. We have $N_{1} \subset N$ and $N_{1}$ is obtained from $N_{0}$ by succesive cell attachments corresponding to the points in $\mathrm{Crit}^{N}(f)$. In particular, because $P$ and $Q$ are consecutive we have the cofibration sequences coming from the successive passage through these two critical points $S^{q-1} \stackrel{j_{Q}}{\rightarrow} N^{\prime} \rightarrow N^{\prime \prime}$, $S^{p-1} \stackrel{j_{P}}{\rightarrow} N^{\prime \prime} \rightarrow N^{\prime \prime \prime} \subset N_{1}$ (where $N^{\prime}$ can be viewed as the union of $N_{0}$ and all the handles associated to critical points $(\in N)$ appearing before $Q$ in the negative gradient flow). By a slight abuse of terminology, we denote by $H\left(j_{P}, j_{Q}\right)$ the relative Hopf invariant of $j_{P}$ and $j_{Q}$ composed with the inclusion $\Sigma^{q} \wedge\left(\Omega N^{\prime \prime \prime}\right)^{+} \hookrightarrow \Sigma^{q} \wedge \Omega M^{+}$.

Here is the main result of this section.

Theorem 1. We have $H\left(j_{P}, j_{Q}\right)=h(P, Q)$.

Remark 2.1. a. We have not been explicit enough in the orientation choices for the Hopf invariants. Therefore, the formula in the statement above should be interpreted as equality up to sign.

b. It has been shown in [5] that in the compact context we have the equality in Theorem 1 after one suspension. The proof of Theorem 1 below is new, more general and leads to a stronger result. It is also more satisfying conceptually as it distinguishes better the purely homotopical part (Proposition 2.2) from the rest of the argument. Moreover, it is also more similar in spirit to the proof of the result in [10] which it extends.

c. To be able to use Theorem 1 to compute homotopically the extended Morse complex $C_{N}^{I}(f)$ one needs to know the relative cell decomposition 
induced by $f$ on an index pair $\left(N_{1}, N_{0}\right)$ of $I_{N}(f)$. This is somewhat unsatisfactory because considerable information on the function $f$ is already necessary to know this decomposition. However, we will see later that, under certain minimality assumptions, this complex can be determined from only the homotopy type of such a pair $\left(N_{1}, N_{0}\right)$.

d. There is a significant advantage in knowing the Thom bordism map $h(P, Q)$ with respect to only the cobordism one: this map allows one to relate properties of $Z(P, Q)$ to global properties of $M$. Notice also that this bordism map contains finer homotopical data than that provided by Conley's connection map associated to the attractor-repellor pair in question.

The proof of Theorem 1 is contained in the first two parts of this section. The first step is purely homotopical and is contained in 32.1 . The rest of the proof is in $\$ 2.2$. The last part of the section contains a number of applications indicating certain rigidity properties of the extended Morse complexes.

\subsection{Hopf invariants as relative attaching maps.}

This sub-section is of a purely homotopical nature and can be read independently of the rest of the paper. The first paragraph contains the main homotopical tool, Proposition 2.2, needed to prove Theorem 11. It shows that relative Hopf invariants can be read out of certain natural relativeattaching maps. The second paragraph discusses some direct consequences of this result. In particular we introduce Hopf complexes which are a homotopical analogue of the extended Morse complexes. In the third paragraph we present some immediate consequences concerning the structure of Thom spaces and the detection of non-smoothable Poincaré duality spaces.

We will work here in the pointed category of spaces with the homotopy type of finite $C W$-complexes and will assume, as in $\$ 1.3$, some familiarity with the the language and the basic results in [14.

\subsubsection{Cone-decompositions and Hopf invariants.}

Consider a pair $\left(X, X_{0}\right)$ of $C W$-complexes. A cone-decomposition of length $k$ of $X$ relative to $X_{0}$ is a sequence of cofibrations sequences

$$
A_{i} \stackrel{j_{i}}{\rightarrow} X_{i} \rightarrow X_{i+1}
$$

with0 $\leq i<k$ such that $X_{k} \simeq X$ and for each $i>0 A_{i}$ is a suspension. Denote by $\delta(i+1, i): A_{i+1} \rightarrow \Sigma A_{i}$ the relative attaching maps obtained by composing $j_{i+1}$ with the connectant $\delta_{i}: X_{i} \rightarrow \Sigma A_{i}$.

Consider the path-loop fibration $\mathcal{P}_{X}: \Omega X \rightarrow P X \rightarrow X$. By pulling back this fibration over the push-out squares

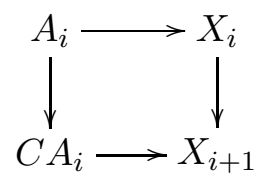

we obtain push-out squares [3] 


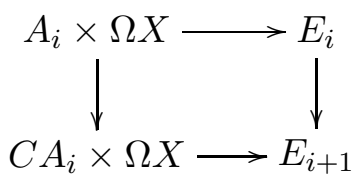

where $E_{i}$ is the total space of the fibration of basis $X_{i}$ obtained by pull-back over the inclusion $X_{i} \rightarrow X$ from $\mathcal{P}_{X}$. By collapsing $\Omega X$ to a point in each corner of the previous push-out we obtain a homotopy cofibration sequence

$$
A_{i} \wedge(\Omega X)^{+} \stackrel{j_{i}^{\prime}}{\rightarrow} E_{i} / \Omega X \rightarrow E_{i+1} / \Omega X
$$

We have used here the formula $(\Sigma T \times K) / K \simeq \Sigma T \wedge K^{+}$. Of course, we have one such cofibration sequence for each $i, 0 \leq i<k$. Each cofibration sequence (2) can be extended one step to the right thus producing a connectant $\delta_{i}^{\prime}: E_{i+1} / \Omega X \rightarrow \Sigma A_{i} \wedge \Omega X^{+}$. Let $\delta^{\prime}(i+1, i)$ be the composition $j_{i+1}^{\prime} \circ \delta_{i}^{\prime}$. In other words, $\delta^{\prime}(i+1, i)$ is the relative attaching map coming out of two succesive cofibration sequences (2).

Proposition 2.2. The map $\delta^{\prime}(i+1, i)$ and the following composition

$$
\begin{aligned}
A_{i+1} \wedge & \Omega X^{+} \stackrel{\left(s \circ H\left(j_{i+1}, j_{i}\right)\right) \wedge i d}{\longrightarrow} \Sigma A_{i} \wedge \Omega X^{+} \wedge \Omega X^{+} \stackrel{i d \wedge \nu}{\longrightarrow} \Sigma A_{i} \wedge \Omega X^{+}
\end{aligned}
$$

coincide up to homotopy. Here $s$ is the inclusion of $X_{i+1}$ into $X$ and $\nu$ is induced by the multiplication $\Omega X \times \Omega X \rightarrow \Omega X$.

Proof. Consider the cube

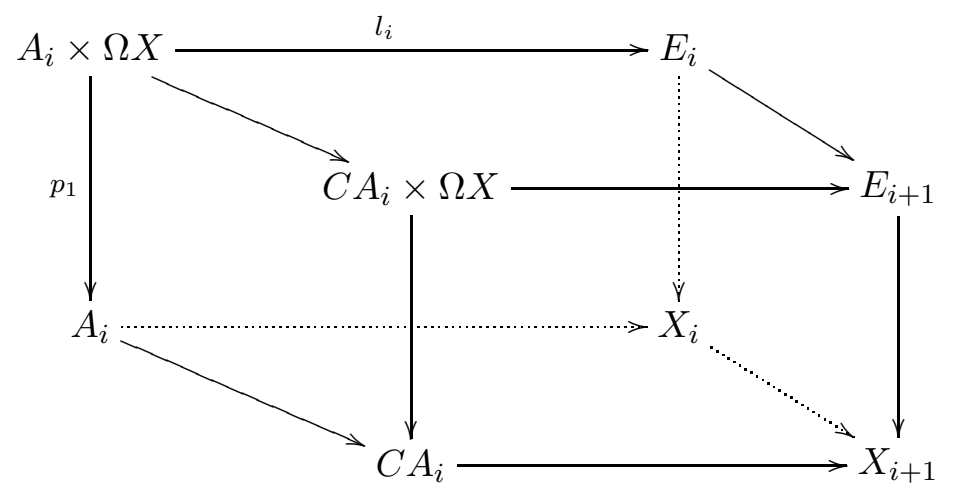

As discussed before this has a push-out as bottom and top squares and has pull-backs as lateral faces. It is obtained by pull-back from the pathloop fibration $\mathcal{P}_{X}$ and by making use of the inclusion $X_{i+1} \hookrightarrow X$. Consider 
now the following diagram

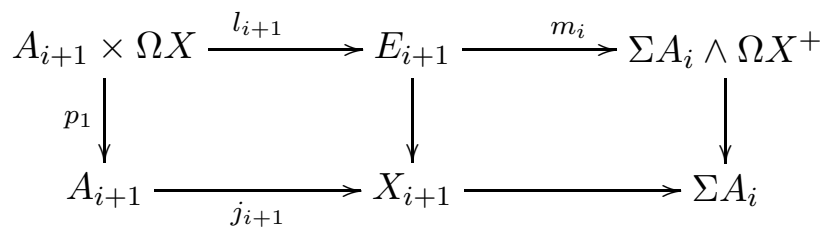

Here the square at the left corresponds to the square at the back of the cube (3) for the index $i+1$ and the square at the right is obtained by taking cofibers in the square at the right of (3) for index $i$. The composition $m_{i} \circ l_{i+1}$ is clearly null-homotopic when restricted to $* \times \Omega X \hookrightarrow A_{i+1} \times \Omega X$. Therefore, the map $\delta^{\prime}(i+1, i)$ is obtained from this composition by collapsing to a point $\Omega X$ in the domain and the image of $l_{i+1}$. Let $r: A_{i+1} \longrightarrow$ $A_{i+1} \times \Omega X \stackrel{l_{i+1}}{\longrightarrow} E_{i+1}$ be such that the first map in the composition is the inclusion on the first component. The map $l_{i+1}$ factors in the following way

$$
l_{i+1}: A_{i+1} \times \Omega X \stackrel{r \times i d}{\longrightarrow} E_{i+1} \times \Omega X \stackrel{\nu^{\prime}}{\longrightarrow} E_{i+1}
$$

where the map $\nu^{\prime}$ is the holonomy of the homotopy fibration $E_{i+1} \rightarrow X_{i+1} \rightarrow$ $X$ and is well defined up to homotopy.

We recall that for an arbitrary fibration $F \rightarrow E \rightarrow B$. the holonomy is obtained by transforming $F \rightarrow E$ into a fibration $\mathcal{F}$ and taking the pull-back of $\mathcal{F}$ over the inclusion $F \hookrightarrow E$. The fact that the composition $F \rightarrow E \rightarrow B$ is trivial implies that the total space of $\mathcal{F}$ is homotopy equivalent to the product $F \times \Omega B$ and the holonomy $\nu_{\mathcal{F}}: F \times \Omega B \rightarrow F$ is the resulting map. If $E$ is contractible, then $\nu: \Omega B \times \Omega B \rightarrow \Omega B$ is, up to homotopy, the usual multiplication.

Here is the justification of the factorization in (5). Up to homotopy, the map $j_{i+1}$ can be written as the composition $A_{i+1} \stackrel{r}{\longrightarrow} E_{i+1} \longrightarrow X_{i+1}$. This means that $l_{i+1}$ is the composition of $\nu^{\prime}$ with the top map in the pull-back diagram

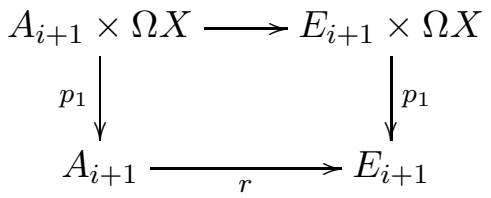

but this map is $r \times i d$ (the diagram is obtained immediately by transforming $E_{i+1} \rightarrow X_{i+1}$ into a fibration and pulling back over the composition $A_{i+1} \stackrel{r}{\rightarrow}$ $\left.E_{i+1} \rightarrow X_{i+1}\right)$. 
The proof of the proposition is now reduced to showing the commutativity of the following diagram

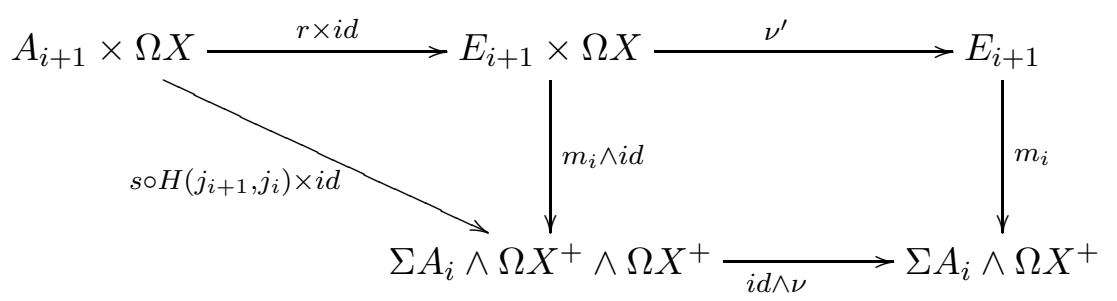

The commutativity of the triangle on the left in (6) follows immediately from the fact that the map $m_{i}$ appears in the following diagram in which all squares are pull-backs (and by performing the "holonomy" construction on the two fiber inclusions represented by the two vertical arrows on the left of the diagram).

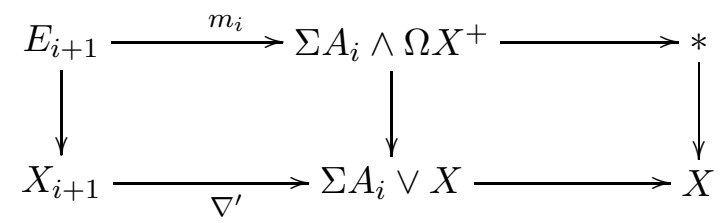

Here the map $\nabla^{\prime}$ is the composition $X_{i+1} \stackrel{\nabla}{\longrightarrow} \Sigma A_{i} \vee X_{i+1} \hookrightarrow \Sigma A_{i} \vee X$. In turn, to verify (7) is is enough to compare the cube below, which is obtained by pull-back from the fibration $\mathcal{P}_{X}$, with the similar cube (3).

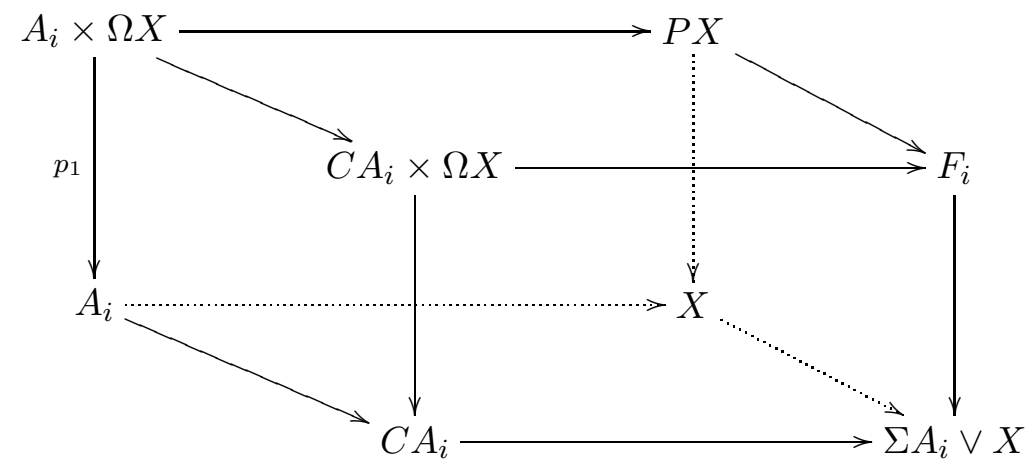

The map $A_{i} \rightarrow X$ is the inclusion and is clearly null-homotopic. This shows that the square on the bottom is indeed a (homotopy) push-out. This cube also explicits the identification of the homotopy fibre $F_{i}$ of $\Sigma A_{i} \vee X \rightarrow X$ with $\Sigma A_{i} \wedge \Omega X^{+}$which is given by the homotopy equivalence of the cofibres of the oblique maps in the top square. The cube (3) maps into (8) the maps defined on each corner being induced by the inclusions on the bottom. The squares at the left in each of these two cubes being the same (and the respective map being the identity) we obtain the claim in (7).

We are now left with verifying the commutativity of the square in (6). In view of (7) we have the following commutative diagram in which the 
columns are pull-backs of $\mathcal{P}_{X}$

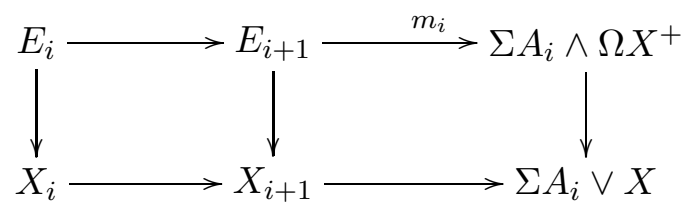

As in the construction of the holonomy of an arbitrary principal fibration we may pull-back all the fibrations in (9) over the projections thus getting a new commutative square relating the various holonomies

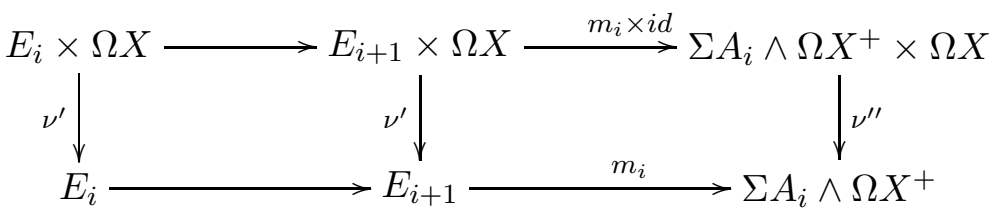

The desired commutativity now follows by noticing that after collapsing to a point $* \times \Omega X$ in the top row of (10) we obtain a cofibration sequence and moreover $\nu^{\prime \prime}$ gives precisely $i d \wedge \nu$ after this operation. This last fact is seen by constructing holonomies over the vertical maps in (8) to express the holonomy $\nu^{\prime \prime}$.

In the remainder of this subsection we discuss some direct, useful homotopical consequences of this proposition. The proof of Theorem 11 is continued in $\$ 2.2$.

\subsubsection{Hopf complexes.}

Assume that $\left(X, X_{0}\right)$ is a relative $C W$-complex with a fixed cell decomposition. Suppose that $X \rightarrow Y$ is a fixed map and $Y$ is simply connected. Denote the set of $i$-cells in the cell- decomposition of $\left(X, X_{0}\right)$ by $\mathrm{Cell}_{i}$. Let $I=\left(a_{1}<a_{2}<\ldots\right) \subset \mathbf{N}$ be such that if $\mathrm{Cell}_{i} \neq \emptyset$ then $i \in I$. Define $\left(C^{I}\left(X, X_{0}\right), d^{I}\right)$ by letting $C^{I}\left(X, X_{0}\right)$ to be the free $\pi_{*}^{S}\left(\Omega Y^{+}\right)$-module generated by $C e l l_{*}$ and if $|e|=a_{i}, e \in C e l l_{*}$, let $d^{I}(e)=$ $\sum_{f \in \operatorname{Cell}_{a_{i-1}}}[H(e, f)] f$ with $[H(e, f)]$ the stable homotopy class of the relative Hopf invariant $H\left(j_{e}, j_{f}\right)$ of the attaching map $j_{e}$ of the cell $e$ relative to the attaching map $j_{f}$ of the cell $f$ viewed inside $\pi_{*}^{S}\left(\Omega Y^{+}\right)$by making use of the map $X \rightarrow Y$. By taking a look to the definition of Hopf invariants in $\$ 1.3$ we see that a special convention is necessary for the coefficients $[H(e, f)]$ if the dimension of the cell $f$ is 0 . We distinguish two cases. First, if $\operatorname{dim}(e)=1$ we let $\delta(e, f)=$ the coefficient of $f$ in the differential of $e$ in the cellular complex associated to the fixed cell-decomposition of the pair $\left(X, X_{0}\right)$. We put $H(e, f)=\delta(e, f) \in \pi_{0}^{S}\left(\Omega Y^{+}\right)=\mathbf{Z}$. If $\operatorname{dim}(e)=p \geq 2$ the attaching map of the cell $e$ is of the form $S^{p-1} \rightarrow X_{0} \amalg \ldots \amalg f$. We take $H(e, f)$ to be 0 if the image of $S^{p-1}$ is disjoint from $f$ and $H(e, f)$ is the adjoint of the map $e / \partial e \rightarrow Y$ if the image of $S^{p-1}$ equals $f$. 
Corollary 2.3. With the notations above $\left(C^{I}\left(X, X_{0}\right), d^{I}\right)$ is a chain complex. A complex of this type will be called a Hopf complex of $\left(X, X_{0}\right)$ with coefficients in $\pi_{*}^{S}\left(\Omega Y^{+}\right)$.

Proof. Apply Proposition 2.2 to express the relevant Hopf invariants in terms of relative attaching maps. Use the fact that the composition of any two succesive relative attaching maps is null-homotopic to deduce relations among the relevant Hopf invariants. After stabilization, these relations are precisely those needed to show $\left(d^{I}\right)^{2}=0$.

Remark 2.4. a. The Hopf complexes as constructed above are closely related to the twisted chain complexes of Baues [1]. In fact, $\left(C^{I}\left(X, X_{0}\right), d^{I}\right)$ can be identified with a chain complex that is easely deduced from this twisted complex. We will not explicit this identification here. It is immediately implied by the fact that the partial suspension of [1] is also given by the following construction. Assume that $A \stackrel{f}{\longrightarrow} B \vee Y$ is a map of pointed $C W$ complexes which is null on $Y$. Then the partial suspension of $f$ is homotopic to the composition $i \circ \Sigma f^{\prime}$. Here $f^{\prime}$ is the unique lift (up to homotopy) of $f$ to the homotopy fibre of $B \vee Y \longrightarrow Y$ and $i$ is the fibre inclusion in the homotopy fibration $\Sigma B \wedge \Omega Y^{+} \rightarrow \Sigma B \vee Y \rightarrow Y$.

b. Smaller is the index set used in defining a Hopf complex $C^{I}\left(X, X_{0}\right)$, more information is contained in the differential of this complex. Indeed, enlarging this index set changes the respective complex by possibly truncating the differential. Moreover, because $Y$ is simply-connected we notice that $C^{\mathbf{N}}\left(X, X_{0}\right)$ is simply the cellular complex associated to the fixed relative cell decomposition of $\left(X, X_{0}\right)$ tensored with $i d_{\pi_{*}^{S}\left(\Omega Y^{+}\right)}$.

It is natural to compare Hopf complexes.

Assume $\left(X^{\prime}, X\right)$ is a relative $C W$-complex. Fix two different relative celldecompositions for $\left(X^{\prime}, X\right)$ and a map $X^{\prime} \rightarrow Y$ with $Y$ simply connected. Let $C^{I}\left(X^{\prime}, X\right)$ and $C_{1}^{I}\left(X^{\prime}, X\right)$ be the two Hopf complexes with coefficients in $\pi_{*}^{S}\left(\Omega Y^{+}\right)$respectively associated to these two cell decompositions.

Lemma 2.5. There is a chain equivalence of the two cellular complexes associated to the two cell decompositions of $\left(X^{\prime}, X\right)$ which extends to a chain equivalence of $C^{I}\left(X^{\prime}, X\right)$ and $C_{1}^{I}\left(X^{\prime}, X\right)$ whenever these Hopf complexes are both defined. In particular, if both cell decompositions are minimal in the sense that in each dimension $k$ they have the same number of cells and this number equals $r k\left(H_{k}\left(X^{\prime}, X ; \mathbf{Z}\right)\right)$, then the Hopf complexes above are isomorphic. If such minimal cell-decompositions exist, then the corresponding Hopf complex that appears for $I=I_{\min }=\left\{k: H_{k}\left(X^{\prime}, X ; \mathbf{Z}\right) \neq 0\right\}$ will be denoted by $\left(\mathcal{C}_{\min }\left(X^{\prime}, X\right), d_{m i n}\right)$ and is well defined up to isomorphism.

Proof. Assume $\vee S^{k} \rightarrow X^{(k)} \rightarrow X^{(k+1)}$ and $\vee S_{1}^{k} \rightarrow X_{1}^{(k)} \rightarrow X_{1}^{(k+1)}$ are the cofibration sequences in the two cell decompositions. We have that $X^{(0)}$, $X_{1}^{(0)}$ are equal to the disjoint union of $X$ and some points. For some $m \in \mathbf{N}$ 
we have $X^{(m)} \simeq X_{1}^{(m)} \simeq X^{\prime}$. By restricting this homotopy equivalence to each of the skeleta we get maps $\phi_{k}: X^{(k)} \rightarrow X_{1}^{(k)}$ that commute with the obvious inclusions and restrict to the identity on $X$. We obtain the commutative diagram below with the top and bottom rows cofibration sequences and where the existence of the doted arrow remains to be discussed.

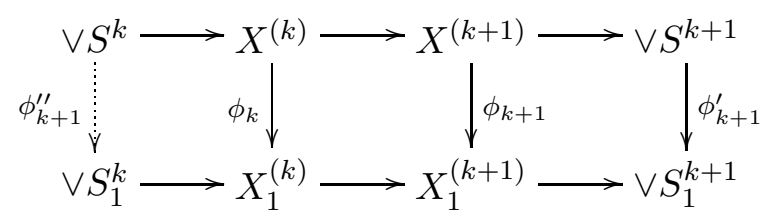

It is a standard fact that the maps $\phi_{t}^{\prime}$ induce a a chain equivalence of cellular complexes $\phi=H_{*}\left(\phi_{*}^{\prime}\right)$. There exists always a map $\phi_{k+1}^{\prime \prime}$ which is a desuspension of $\phi_{k+1}^{\prime}$. If $k \geq 2$ this desuspension is uniquely defined and we first notice that, under this same assumption, it does make commute the left square of the diagram. This is quite immediate if $X^{\prime}$ is simply connected and therefore both $X^{(k)}$ and $X_{1}^{(k)}$ are so. Indeed, the composition $\pi_{k}\left(\vee S^{k}\right) \approx$ $H_{k}\left(\vee S^{k}\right) \approx H_{k+1}\left(X^{(k+1)}, X^{k}\right) \approx \pi_{k+1}\left(X^{(k+1)}, X^{(k)}\right) \rightarrow \pi_{k}\left(X^{(k)}\right)$ describes precisely the attaching maps of the $k+1$ cells in the first cell-decomposition and the analogue statement is valid, of course, for the second decomposition. But all the maps in these compositions commute with the map induced in relative homology by the pair $\left(\phi_{k+1}, \phi_{k}\right)$. If $X^{\prime}$ is not simply-connected (but still $k \geq 2$ ) one needs to first apply the above argument to the universal covering $\tilde{X}^{\prime}$ relative to the cell-decompositions obtained by lifting those given for $X^{\prime}$.

Our purpose now is to show that the map of complexes $\phi^{I}: C^{I}\left(X^{\prime}, X\right) \rightarrow$ $C_{1}^{I}\left(X^{\prime}, X\right)$ defined by $\phi \otimes i d_{\pi_{*}^{S}\left(\Omega Y^{+}\right)}$is a chain map. This is immediate if the commutativity in the left two squares of the diagram (11) would hold for all $k$ because all the relevant constructions (co-actions, lifts, etc) would be comparable by maps induced by the $\phi_{k+1}^{\prime \prime}$ 's. However, in general, this commutativity is not valid for $k \leq 1$. We first assume that $2 \in I$ and let $q$ be the first index in $I$ that is bigger than 2 . It is easy to see, due to the relation between Hopf complexes and cellular complexes, that for dimensions $t \leq 2$ the maps $\phi^{I}$ commutes with the differentials in the respective Hopf complexes. This also happens for $t>q$, as indicated above, because of the commutativity of the diagram (11). Moreover, the commutativity in the left square of (11) is valid for $k=q-1$. In constructing the relative Hopf invariants of a cell of dimension $q$ and one of dimension 2 , besides the attaching maps for the cell of dimension $q$, we also need the maps $X^{(2)} \rightarrow$ $X^{(2)} \vee\left(\vee S^{2}\right) \rightarrow Y \vee\left(\vee S^{2}\right)$ where the first map in this composition is the co-action and the last is induced by inclusion. It is easy to see that the remaining commutativity necessary for $\phi^{I}$ to be a chain morphism reduces to showing that the square below commutes. 


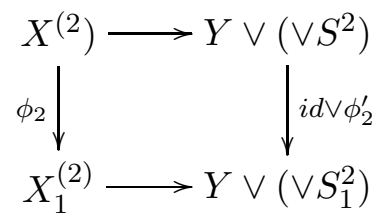

Because $Y$ is simply connected, this commutativity is satisfied if its homology version is true which is immediate. The same argument applies to the case when $2 \notin I$ but $1 \in I$ and shows that $\phi^{I}$ is a chain morphism.

Because $\phi$ is a chain equivalence $\phi^{I}$ is forced to be one also. The reason is that the differential in the cellular complex associated to a cell decomposition of $\left(X^{\prime}, X\right)$ is contained in the differential of each Hopf complex $C^{I}\left(X^{\prime}, X\right)$ which is defined.

The last claim in the statement is obvious because for a minimal cell decomposition the cellular differential is trivial and therefore, as the comparison morphism $\phi^{I}$ is a chain equivalence, it is in fact an isomorphism.

Remark 2.6. A condition that insures the existence of a minimal cell decomposition as in the lemma above is the following: $\left(X^{\prime}, X\right)$ has torsion free integral homology and both $X^{\prime}$ and $X$ are simply-connected.

Here is a relation between relative Hopf invariants and a certain AtiyahHirzeburch spectral sequence. We will only formulate this consequence in the setting of the Remark 2.6.

Therefore, $\left(X, X_{0}\right)$ is a $C W$-pair with both $X_{0}$ and $X$ simply-connected and such that the integral homology of the pair is torsion free. We consider Hopf complexes with coefficients in $\pi_{*}\left(\Omega X^{+}\right)$(in other words we take $Y=$ $X$ and the relevant map $X \rightarrow Y$ is the identity). We have a ring map $h: \pi_{*}^{S}\left(\Omega X^{+}\right) \rightarrow H_{*}\left(\Omega X^{+} ; \mathbf{Z}\right)$. We can change the coefficents in $\mathcal{C}_{\min }\left(X, X_{0}\right)$ by using this map and we denote by $\left(\check{\mathcal{C}}_{\text {min }}\left(X, X_{0}\right), \check{d}_{\text {min }}\right)$ the resulting chain complex of $H_{*}\left(\Omega X^{+} ; \mathbf{Z}\right)$-modules. Let $\left(\mathcal{E}_{p q}^{r}, D^{r}\right)$ be the Atiyah-HirzebruchSerre spectral sequence of the relative fibration $\mathcal{P}^{\prime}$ of basis $\left(X, X_{0}\right)$ induced from the fibration $\Omega X \rightarrow P X \rightarrow X$ with

$$
\mathcal{E}^{2}=H_{*}\left(X, X_{0} ; \pi_{\odot}^{S}\left(\Omega X^{+}\right)\right) \approx H_{*}\left(X, X_{0} ; \mathbf{Z}\right) \otimes \pi_{\odot}^{S}\left(\Omega X^{+}\right)
$$

and which converges to $\pi_{*+\odot}^{S}$. Similarly, we let $\left(E_{p q}^{r}, d^{r}\right)$ be the homology Serre spectral sequence of the fibration $\mathcal{P}^{\prime}$.

Corollary 2.7. In the setting above assume that $a, b \in \mathbf{N}$ are such that $H_{k}\left(X, X_{0} ; \mathbf{Z}\right)=0$ for $a<k<b, b-a>1$. If $H_{k}\left(X, X_{0} ; \mathbf{Z}\right) \neq 0$ for $k \in\{a, b\}$, then

(i) $\left.d_{\text {min }}\right|_{\text {Cell }_{b}} \rightarrow \pi_{*}^{S}\left(\Omega X^{+}\right)<$Cell $_{a}>$ is identified to $\left.D^{a-b}\right|_{\mathcal{E}_{a 0}^{b-a}}$.

(ii) $\left.\check{d}_{\text {min }}\right|_{\text {Cell }} \rightarrow H_{*}\left(\Omega X^{+} ; \mathbf{Z}\right)<$ Cell $_{a}>$ is identified to $\left.d^{a-b}\right|_{E_{a 0}^{b-a}}$. 
Proof. This follows by noting that the elements in $\mathcal{E}_{a 0}^{2}$ (repspectively in $E_{a 0}^{2}$ survive to $\mathcal{E}_{a 0}^{a-b}$ (resp. $E_{a 0}^{a-b}$ ) for dimensionality reasons and then by recalling the definition of the two spectral sequences considered (as described for example in [28]) and applying the proposition.

Remark 2.8. The second point of the corollary is particularly useful because it allows one to compute the homology version of the minimal Hopf complex only in terms of the Serre spectral sequence of the path-loop fibration of $X$. In turn, this spectral sequence is generally very well understood. If we are interested in a non minimal Hopf complex or even one Hopf complex that comes from a non-minimal cell decomposition it is still possible to estimate it, in a rather obvious way, by using these spectral sequences but we will not pursue further this issue here.

\subsubsection{Duality.}

Assume that $\xi: X \longrightarrow B G$ is the classifying map of a fiber bundle of rank $m$, of basis $X$ and of structural group $G$. Let $E_{S}^{\xi}(X)$ be the total space of the associated spherical fibration and let $T^{\xi}(X)$ be the respective Thom

space. Fix also the cone-decomposition $A_{i} \stackrel{j_{i}}{\rightarrow} X_{i} \rightarrow X_{i+1}, 0 \leq i<k$ as in (1) together with the relative attaching maps $\delta(i+1, i): A_{i+1} \rightarrow \Sigma A_{i}$.

Corollary 2.9. The Thom space $T^{\xi}(X)$ admits a cone decomposition

$$
\Sigma^{m} A_{i} \stackrel{j_{i}^{\prime \prime}}{\rightarrow} T^{\xi}\left(X_{i}\right) \rightarrow T^{\xi}\left(X_{i+1}\right)
$$

such that the corresponding attaching maps $\delta^{\prime \prime}(i+1, i): \Sigma^{m} A_{i+1} \rightarrow \Sigma^{m+1} A_{i}$ verify (up to sign) $\delta^{\prime \prime}(i+1, i)=\Sigma^{m} \delta(i+1, i)+\bar{\nu} \circ \Omega \xi \circ \Sigma^{m} H\left(j_{i+1}, j_{i}\right)$. Here $\bar{\nu}: \Sigma A_{i} \wedge S^{m-1} \wedge G \rightarrow \Sigma A_{i} \wedge S^{m-1}$ is induced by the action of $G$ on $S^{m-1}$ and $\Omega \xi: \Sigma A_{i} \wedge S^{m-1} \wedge \Omega X \rightarrow \Sigma A_{i} \wedge S^{m-1} \wedge G$.

Proof. Notice that the exact same constructions as in the proof of the proposition can be performed for the fibration $\xi$ instead of the fibration $\mathcal{P}_{X}$. Of course, as $\mathcal{P}_{X}$ is universal, the map $\Omega X \rightarrow S^{m-1}$ induces comparison maps between the diagrams obtained for $\mathcal{P}_{X}$ and those for $\xi$. Therefore, the argument in the proposition describes also the relative attaching maps coming from a cone-decomposition $A_{i} \wedge\left(S^{m-1}\right)^{+} \rightarrow E_{S}^{\xi}\left(X_{i}\right) / S^{m-1} \rightarrow$ $E_{S}^{\xi}\left(X_{i}\right) / S^{m-1}$. It is now immediate to see that, by collapsing each such cofibration sequence into the cofibration $A_{i} \rightarrow X_{i} \rightarrow X_{i+1}$ one gets cofibration sequences $A_{i} \wedge S^{m} \rightarrow T^{\xi}\left(X_{i}\right) \vee S^{m} \rightarrow T^{\xi}\left(X_{i+1}\right) \vee S^{m}$. We then collapse to a point the sphere $S^{m}$ in the last two terms and obtain the cofibration sequences of the statement and the claimed formula for the relative attaching maps.

Remark 2.10. a. The formula in this corollary also appears in Dula in [8].

b. Of particular interest is the case when the spaces $A_{i}$ are spheres of dimensions, respectively, $a_{i}$. Then $H\left(j_{i+1}, j_{i}\right) \in \pi_{a_{i+1}}\left(\Sigma^{a_{i}+1}(\Omega X)^{+}\right)$and the stable difference $\delta^{\prime \prime}(i+1, i)-\delta(i+1, i)$ becomes equal to $J_{G}^{a_{i}}\left(\Omega \xi \circ H\left(j_{i+1}, j_{i}\right)\right)$ 
where $J_{G}^{a_{i}}: \pi_{a_{i+1}-a_{i}-1} \Omega^{a_{i}} \Sigma^{a_{i}} G \rightarrow \pi_{a_{i+1}-a_{i}-1}^{S}$ is the factor of the $J_{G}$ homomorphism (as defined for example in [30] and also see below) $J_{G}: \pi_{*} G \rightarrow \pi_{*}^{S}$.

c. The statement of Corollary 2.9 is clearly also valid if applied directly to spherical fibrations (and not only to bundles).

Assume now that $X$ is a Poincaré duality space and let $\zeta$ be its Spivak stable normal bundle 27.

We have a ring map $s: \pi_{*}^{S}\left(\Omega X^{+}\right) \rightarrow \pi_{*}^{S}$. Assume also that $X$ is simplyconnected and does not have any torsion in homology. Then $(X, *)$ has a minimal cell-decomposition in the sense of the lemma above. Denote by $\mathcal{C}_{\min }(X)$ the Hopf complex associated to such a minimal cell-decomposition with coefficents in $\pi_{*}^{S}\left(\Omega X^{+}\right)$. Let $\left(\overline{\mathcal{C}}_{\min }(X), \bar{d}\right)$ be the chain complex with coefficients in $\pi_{*}^{S}$ obtained from $\mathcal{C}_{\min }(X)$ by changing the coefficients via $s$. The coefficients of the differential in this chain complex are homotopy classes of relative attaching maps of consecutive pairs of cells. Finally, let $\left(\overline{\mathcal{C}}_{\text {min }}^{\prime}(X), \bar{d}^{\prime}\right)$ be the following $\pi_{*}^{S}$-chain complex. As $\pi_{*}^{S}$-modules we have isomorphisms $\left(\overline{\mathcal{C}}_{\text {min }}^{\prime}(X)\right)_{n-k} \approx\left(\overline{\mathcal{C}}_{\text {min }}(X)\right)_{k}$ and if $x$ is a generator of $\left(\overline{\mathcal{C}}_{\text {min }}(X)\right)_{k}$ we denote by $x^{*}$ the corresponding generator of $\left(\overline{\mathcal{C}}_{\text {min }}^{\prime}(X)\right)_{n-k}$; by definition, the differential verifies $\bar{d}^{\prime}\left(x^{*}\right)=\sum \bar{d}^{\prime}\left(x^{*}, y^{*}\right) y^{*}$ with $\bar{d}^{\prime}\left(x^{*}, y^{*}\right)=$ $\bar{d}(y, x)+J_{B F}^{|x|}(\Omega \zeta \circ H(y, x))$. Here $y$ and $x$ represent cells in the minimal celldecomposition that have consecutive indexes in $\left\{k \in \mathbf{N}: H_{k}(X: \mathbf{Z}) \neq 0\right\}$; $H(y, x)$ is the respective relative Hopf invariant; $B F$ is the classifying space of spherical fibrations (see also Remark 2.10) and $\bar{d}(y)=\sum \bar{d}(y, x) x$. We also recall the definition of the $J$-homomorpphism [30]. This is a homomorphism $J_{S O}: \pi_{k}(\mathbf{S O}) \rightarrow \pi_{k}^{S}$ that associates to the homotopy class of a map $a: S^{k} \rightarrow S O$ the homotopy class of a map obtained as follows. First, for $q$ sufficiently big, let $b: S^{q-1} \times S^{k} \stackrel{i d \times a}{\rightarrow} S^{q-1} \times \mathbf{S O}(q) \stackrel{\mu}{\rightarrow} S^{q-1}$ where $\mu$ is the action. Define $J_{S O}(a)$ to be the stable homotopy class of the restriction of $\Sigma b$ to $S^{q+k} \subset \Sigma\left(S^{q-1} \times S^{k}\right)$. The morphism $J_{S O}$ has a stable target. Because of that it factors as $\pi_{k}(\mathbf{S O}) \rightarrow \pi_{k}(\Omega \Sigma \mathbf{S O}) \ldots \pi_{k}\left(\Omega^{q} \Sigma^{q} \mathbf{S O}\right) \rightarrow \ldots \pi_{k}^{S}(\mathbf{S O}) \stackrel{J_{S O}^{\prime}}{\rightarrow}$ $\pi_{k}^{S}$. We denote by $J_{S O}^{q}$ the restriction of $J_{S O}^{\prime}$ to $\Omega^{q} \Sigma^{q} \mathbf{S O}$. The same morphism can be defined in an analogue fashion for other groups $G$ that have the property to act on homotopy spheres. The corresponding homomorphisms are denoted by $J_{G}$ and $J_{G}^{q}$.

Corollary 2.11. In the setting above ( $X$ simply-connected and whithout torsion in homology) the two $\pi_{*}^{S}$-chain complexes $\left(\overline{\mathcal{C}}_{\text {min }}^{\prime}(X), \bar{d}^{\prime}\right)$ and $\left(\overline{\mathcal{C}}_{\text {min }}(X), \bar{d}\right)$ are isomorphic. If the stable spherical normal fibration of $X$ admits a reduction to BG (for example, BPL or BSO etc.), then

$$
\bar{d}^{\prime}\left(x^{*}, y^{*}\right)=+/-\bar{d}(y, x) \bmod \operatorname{Im}\left(J_{B G}^{|x|}\right)
$$

Proof. This follows from Corollary 2.9 and Remark 2.10. Indeed, the Thom space of the Spivak normal stable bundle of $X$ is Spanier-Whitehead dual to $X$ itself. Therefore, the fixed minimal cell decomposition on $X$ induces 
by duality a minimal decomposition for this Thom space. As SpanierWhitehead duality for maps between spheres coincides with stable equality up to sign the relative attaching maps of this decomposition coincide stably with those of the one fixed on $X$. Moreover, as both this decomposition and that provided by Corollary 2.9 are minimal we obtain that the two complexes in question are isomorphic. The second part of the statement is clear given the definition of the $J$-homomorphism.

Remark 2.12. The problem of deciding whether a given Poincaré duality space $X$ admits a PL or smooth structure is one of the central problems of differential topology. Surgery theory provides a solution to this question by reducing it to a two stage process: first, one has to decide whether the Spivak stable normal bundle admits a lift to $B P L$ or, respectively, $B S O$ and, if this is the case, a secondary numerical invariant needs to vanish. The existence of a lift is theoretically the simple step as it reduces to a typical obstruction theory problem. However, in practice, even for spaces $X$ having a small number of cells it is not easy to show that such a lift does not exist. Indeed, the canonical way to produce a lift is to extend it inductively over each skeleton of $X$ and if some non-vanishing obstruction appears at some step in the process one is forced to start back from the bottom. This direct approach has the disadvantage that, to conclude the non-existence of a lift, one has to show that each possible attempt leads to a non-vanishing obstruction. The corollary above provides a shortcut to this process: if for some $x, y$ as in the corollary we do not have the relation $\bar{d}^{\prime}\left(x^{*}, y^{*}\right)=+/-\bar{d}(y, x) \bmod \operatorname{Im}\left(J_{S O}^{|x|}\right)$ for example, then $X$ is not smoothable. The first such example of a Poincaré duality space $X$ with $|x|=0$ was described by Smith in [26] (his proof being different from the method indicated here). Examples with $|x|>0$ have been first described in [5] the proof given there was however not completely homotopical. To the author's knowledge, there is no other general method to construct explicitely non-smoothable Poincaré duality, 1-connected, $C W$-complexes that remain non-smoothable after product with an arbitrary sphere.

\subsection{Relating Hopf invariants and bordism maps.}

The purpose of this sub-section is to relate relative Hopf invariants to the Thom bordism maps associated to connecting manifolds and in this way prove Theorem 1 .

We now return to the setting of this theorem.

As in 2.1 .1 let $\mathcal{P}_{M}$ be the path-loop fibration $\Omega M \rightarrow P M \rightarrow M$. Of course, we have inclusions $N_{0} \hookrightarrow N^{\prime} \hookrightarrow N^{\prime \prime} \hookrightarrow N^{\prime \prime \prime} \hookrightarrow N_{1} \hookrightarrow M$. For any subset $A \subset M$ we can pull-back $\mathcal{P}_{M}$ over $A$ thus getting a new fibration of basis $A$ whose total space will be denoted by $T(A)$. As in (2) we obtain two cofibration sequences $S^{q-1} \wedge \Omega M^{+} \rightarrow T\left(N^{\prime}\right) / \Omega M \rightarrow T\left(N^{\prime \prime}\right) / \Omega M$ and $S^{p-1} \wedge$ $\Omega M^{+} \rightarrow T\left(N^{\prime \prime}\right) / \Omega M \rightarrow T\left(N^{\prime \prime \prime}\right) / \Omega M$ that produce a relative attaching map $\delta: S^{p-1} \wedge \Omega M^{+} \rightarrow S^{q} \wedge \Omega M^{+}$. 
In view of Proposition 2.2 the statement of the theorem is clear if we show that $\delta$ is homotopic to the composition

$$
S^{p-1} \wedge \Omega M^{+} \stackrel{h(P, Q) \wedge i d}{\longrightarrow} S^{q} \wedge \Omega M^{+} \wedge \Omega M^{+} \stackrel{i d \wedge \nu}{\longrightarrow} S^{q} \wedge \Omega M^{+}
$$

where, as in Subsection 2.1, $\nu$ is the multiplication on $\Omega M$.

We denote by $\gamma$ the flow $\gamma(f)$. The first step of the proof is to make some explicit constructions which will allow us to express $\delta$ in terms of some simple index pair of the isolated invariant set $I(P, Q)$ which, we recall, is the union of $P, Q$ and of all the points situated on flow lines of $\gamma$ that join $P$ to $Q$ and are included in $N$.

\subsubsection{A simple index pair of $I(P, Q)$.}

Let

$$
\begin{aligned}
U(P ; \epsilon, \tau) & =\left\{x \in M: \exists T_{x} \in \mathbf{R} \cup\{-\infty, \infty\}, \gamma_{T_{x}}(x) \in\right. \\
& \left.\in D(P, \tau) \cap f^{-1}(f(P)), \quad|f(x)-f(P)| \leq \epsilon\right\}
\end{aligned}
$$

where $D(P, \tau)$ is the closed disk of radius $\tau$ and center $P$. Clearly, for $\epsilon$ and $\tau$ small the set $U(P ; \epsilon, \tau)$ is a manifold (with a boundary with corners) homeomorphic to the $n$-dimensional closed disk. Let $A(P ; \epsilon, \tau)=$ $U(P ; \epsilon, \tau) \cap f^{-1}(f(P)-\epsilon), B(P ; \epsilon, \tau)=U(P ; \epsilon, \tau) \cap f^{-1}(f(P)+\epsilon)$. Then $A(P ; \epsilon, \tau)$ is diffeomorphic to $S^{p-1} \times D^{n-p}$ and $B(P ; \epsilon, \tau)$ is diffeomorphic to $D^{p} \times S^{n-p-1}$. In these identifications $S^{p-1} \times\{0\}$ and $\{0\} \times S^{n-p}$ correspond respectively to the unstable and the stable manifolds of $P$ intersected respectively with $f^{-1}(f(P)-\epsilon)$ and $f^{-1}(f(P)+\epsilon)$. We also denote by $A^{\prime}(P ; \epsilon, \tau)$ the closure of the set $\partial U(P ; \epsilon, \tau)-B(P ; \epsilon, \tau)$. The two diffeomorphisms above can be made quite explicit once Morse coordinates are chosen around $P$. Using such coordinates $(x, y)$ with $f$ given by $-\|x\|^{2}+\|y\|^{2}+f(P)$ there is a diffeomorphism of $A(P ; \epsilon, \tau)$ with a set of the type $\left\{(x, y) \in \mathbf{R}^{p} \times \mathbf{R}^{n-p}\right.$ : $\left.\epsilon \leq\|x\|^{2} \leq \epsilon+\eta,-\|x\|^{2}+\|y\|^{2}=-\epsilon\right\}$. and a similar identification is valid for $B(P ; \epsilon, \tau)$. Moreover, the flow induces a diffeomorphism between $B(P ; \epsilon, \tau)-W_{N}^{s}(P)$ and $A(P ; \epsilon, \tau)-W_{N}^{u}(P)$ which corresponds to the obvious twist map $\left(S^{p-1} \times(0,1]\right) \times S^{n-p-1} \longrightarrow S^{p-1} \times\left(S^{n-p-1} \times(0,1]\right)$. We also need to notice that, once a Morse chart as above is fixed, there are $n$-linearly independent sections of the tangent space of $U(P ; \epsilon, \tau)$ each in the direction of one of the coordinates $x_{i}$ or $y_{j}$. These also provide normal framings to the sets $\operatorname{Int}\left(W_{N}^{u}(P)\right)$ and $\operatorname{Int}\left(W_{N}^{s}(P)\right)$. These framings have the property to be coherent with the decomposition $A(P ; \epsilon, \tau) \approx S^{p-1} \times D^{n-p}$ (and similarly for $B(P ; \epsilon, \tau)$ ) in the sense that they induce the standard framing of $S^{p-1} \times\{0\} \subset S^{p-1} \times D^{n-p}$. In particular, we may see $A(P ; \epsilon, \tau)$ as the total space of a tubular neighbourhood of $S^{u}(P)$. We denote by $\iota_{P}^{-}$ and $\iota_{P}^{+}$the framings of $S^{u}(P)$ in $A(P ; \epsilon, \tau)$ and respectively that of $S^{s}(P)$ in $B(P ; \epsilon, \tau)$. Of course, these framings depend on the choice of a Morse chart but we suppose from now on that such a choice has been made for all critical points of $f$. A different choice will not modify the isomorphism class of these framings (except possibly by a sign). 
An analogue construction is of course possible for the critical point $Q$. As the critical points $P$ and $Q$ are consecutive we may assume (after possibly slightly modifying $f$ ) that for some choice of $\epsilon$ and $\tau$ the sets $U(Q)=$ $U(Q ; \epsilon, \tau), U(P)=U(P ; \epsilon, \tau)$ and $A(P)=A(P ; \epsilon, \tau)$ verify $U(P) \cap U(Q)=$ $\left\{x \in A(P): \gamma_{\mathbf{R}}(x) \cap U(Q) \neq \emptyset\right\}$ (this implies, in particular, that $\epsilon$ is such that $f(Q)+\epsilon=f(P)-\epsilon)$. Moreover, the intersection of $S^{p-1} \times\{0\} \subset$ $A(P)$ and $\{0\} \times S^{n-q-1} \subset B(Q)$ are transverse in $f^{-1}(\epsilon+f(Q))$ (where $B(Q)=B(Q ; \epsilon, \tau)$ and we will use similar notations for the rest of the sets $A, B, A^{\prime}$ associated to $U(P)$ and $\left.U(Q)\right)$. This means that by assuming $\tau$ sufficiently small, the intersection of $A(P)$ and $B(Q)$ is also transverse and, by using the identifications described before for these two sets and letting $K=U(P) \cap U(Q)$, we have $K=A(P) \cap B(Q) \approx D^{q} \times Z(P, Q) \times D^{n-p}$. Clearly, $D^{q} \times Z(P, Q) \times\{0\} \subset S^{u}(P)$ and $Z(P, Q) \hookrightarrow S^{u}(P)$ is normally framed via the framing obtained by projection from $\iota_{Q}^{+}$. We will denote this framing of $Z(P, Q)$ by $\iota^{+}$and we let $W(Z)=D^{q} \times Z(P, Q) \times\{0\} \subset S^{u}(P)$ be the tubular neighbourhood of $Z(P, Q)$ described above. Of course, $\iota^{+}$ identifies $W(Z)$ with the total space of the normal bundle of $Z(P, Q)$ in $S^{u}(P)$. The framing $\iota^{+}$is precisely the one used to construct the bordism class of $Z(P, Q)$.

Let $W^{\prime}=A^{\prime}(Q) \cup(A(P)-K), W^{\prime \prime}=U(Q) \cup A(P), W^{\prime \prime \prime}=U(Q) \cup U(P)$. These three sets are related by cofibration sequences described before the statement of the theorem. They also verify the needed index pairs conditions and it is immediate to see that they can be used in the constructions made at the beginning of the proof by replacing $N^{\prime}, N^{\prime \prime}, N^{\prime \prime \prime}$ by $W^{\prime}, W^{\prime \prime}$ and $W^{\prime \prime \prime}$, respectively. Moreover, $\delta$ is also given as the relative attaching map described in that construction now applied to the spaces $T\left(W^{\prime}\right) / \Omega(M), T\left(W^{\prime \prime}\right) / \Omega M$ and $T\left(W^{\prime \prime \prime}\right) / \Omega M$. Indeed, $\left(W^{\prime \prime \prime}, W^{\prime}\right)$ is an index pair of $I(P, Q)$ and for any other index pair $\left(N^{\prime \prime \prime}, N^{\prime}\right)$ of this invariant set we may choose $s, \tau$ sufficiently small such that the set $(U(Q ; \epsilon, \tau) \cup U(P ; \epsilon, \tau)) \cap f^{-1}([f(Q)-s, f(P)+s])$ (which is diffeomorphic to $U(P) \cup U(Q)$ and has analogue properties) is contained in $N^{\prime \prime \prime}$. This allows one to identify the attaching map constructed in $T\left(N^{\prime \prime \prime}\right)$ with that constructed in $T\left(W^{\prime \prime \prime}\right)$.

\subsubsection{The Thom-Pontryaguin construction and $\delta$.}

Our next purpose is to sufficiently explicit $\delta$ as to show that it is given by the composition in (14). Recall that for any subspace $A \hookrightarrow M$ we denote by $T(M)$ the total space of the fibration of basis $A$ obtained by pull-back from $\mathcal{P}_{M}$. By revisiting (3) (with $A_{i+1}=S^{u}(P)$ and $A_{i}=S^{u}(Q)$ ) we see that $\delta$ is determined by the following composition

$$
\delta^{\prime}: T\left(S^{u}(P)\right) \hookrightarrow T(A(P)) \hookrightarrow T\left(W^{\prime \prime}\right) \stackrel{l}{\longrightarrow} T\left(W^{\prime \prime}\right) / T\left(W^{\prime}\right)
$$

where the first three maps are inclusions and $l: T\left(W^{\prime \prime}\right) \rightarrow T\left(W^{\prime \prime}\right) / T\left(W^{\prime}\right)$ is the projection. Indeed, $\delta^{\prime}$ is immediately seen to be equal to the composition $T\left(S^{u}(P)\right) \rightarrow T\left(S^{u}(P)\right) / \Omega M \stackrel{\delta}{\rightarrow}\left(T\left(W^{\prime \prime}\right) / \Omega M\right) /\left(T\left(W^{\prime}\right) / \Omega M\right)=$ $T\left(W^{\prime \prime}\right) / T\left(W^{\prime}\right)$. At the same time $T\left(W^{\prime \prime}\right) / T\left(W^{\prime}\right)=T(U(Q)) / T\left(A^{\prime}(Q)\right)$. 
It follows that $\delta^{\prime}$ is also equal to the composition

$$
T\left(S^{u}(P)\right) \stackrel{k}{\rightarrow} T(W(Z)) / T(\partial W(Z)) \hookrightarrow T(U(Q)) / T\left(A^{\prime}(Q)\right.
$$

where the map $k$ is projection on the respective quotient and the last map is well defined because $\partial W(Z) \subset A^{\prime}(Q)$. The fibrations producing the total spaces in (17) are all trivial because, one one hand, $A^{\prime}(Q) \subset U(Q) \simeq *$ and on the other $\partial W(Z) \subset W(Z) \subset S^{u}(P) \subset W^{u}(P) \simeq *$. To get to the expression in (14) we need to explicitely use the respective trivializations. Fix a trivialization of the fibration $\mathcal{F}_{W^{u}(P)}$ obtained as a pull-back of $\mathcal{P}_{M}$. It produces the following diagram where all horizontal arrows are the obvious inclusions and the vertical arrows are homeomorphisms

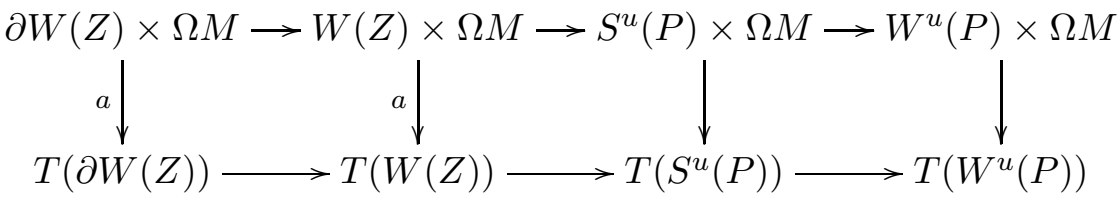

Notice that $W(Z) \subset B(Q) \subset U(Q)$. Therefore, if we fix a trivialization for the fibration induced over $U(Q)$ (from $\mathcal{P}_{M}$ ) we get another similar diagram

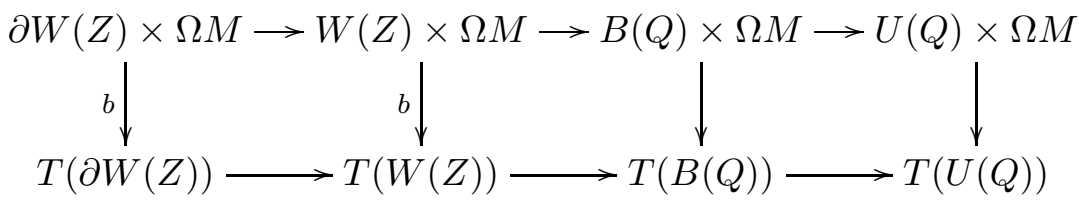

We may consider the composition $c=a \circ b^{-1}$ of the two homeomorphisms $a$ and $b$ appearing respectively in the diagrams (18) and (19). It makes commutative the diagram

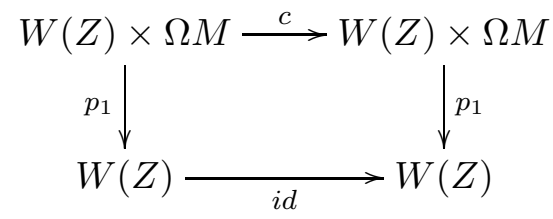

however the key point is that, in general, $c$ is not itself the identity. However, it is easy to see that it is described, up to homotopy, by the following composition

$$
\begin{gathered}
W(Z) \times \Omega M \stackrel{\Delta \times i d}{\longrightarrow} W(Z) \times W(Z) \times \Omega M \stackrel{i d \times p_{2} \times i d}{\longrightarrow} \\
\rightarrow W(Z) \times Z(P, Q) \times \Omega M \stackrel{i d \times j(P, Q) \times i d}{\stackrel{i}{\longrightarrow}} W(Z) \times \Omega M \times \Omega M \stackrel{i d \times \nu}{\longrightarrow} \\
\rightarrow W(Z) \times \Omega M
\end{gathered}
$$

where $p_{2}: W(Z) \rightarrow Z(P, Q)$ is the projection $D^{q} \times Z(P, Q) \rightarrow Z(P, Q)$ and, as before, $\nu$ is the multiplication. The restriction of $c$ to $\partial W(Z)$ is clearly also a homeomorphism and its description is obtained from (20) by restriction (recall that $\left.\partial W(Z)=S^{q-1} \times Z(P, Q) \subset D^{q} \times Z(P, Q)=W(Z)\right)$. 
From (20) and (17) we obtain the following description of the homotopy class of $\delta^{\prime}$

$$
\begin{array}{r}
S^{p-1} \times \Omega M \longrightarrow W(Z) \times \Omega M / \partial W(Z) \times \Omega M \stackrel{\bar{c}}{\longrightarrow} \\
\rightarrow W(Z) \times \Omega M / \partial W(Z) \times \Omega M \longrightarrow U(Q) \times \Omega M / A^{\prime}(Q) \times \Omega M \rightarrow \\
\rightarrow U(Q) \times \Omega M / A(Q) \times \Omega M \stackrel{t}{\longrightarrow} S^{q} \wedge \Omega M^{+}
\end{array}
$$

where $\bar{c}$ is the homeomorphism induced by $c, t$ is the obvious composition $U(Q) \times \Omega M / A(Q) \times \Omega M \rightarrow\left(D^{q} \times D^{n-q} \times \Omega M\right) /\left(S^{q-1} \times D^{n-q} \times \Omega M\right) \rightarrow$ $\left(D^{q} \times \Omega M\right) /\left(S^{q-1} \times \Omega M\right) \rightarrow S^{q} \wedge \Omega M^{+}$and the map preceding $t$ is induced by a homeomorphism deforming $A^{\prime}(Q)$ to $A(Q)$. We did also identify $S^{u}(P)$ to $S^{p-1}$.

Let us denote by $\delta^{\prime \prime}$ the restriction of $\delta^{\prime}$ in (21) to $S^{p-1} \times *$. In view of the description of $c$ in (20), to show that $\delta$ is the composition in (14) it is enough to prove that $\delta^{\prime \prime}$ is precisely the Thom map $h(P, Q)$ associated to the framing $\iota^{+}$and the map $j(P, Q)$. Recall that this Thom map is defined as the composition

$$
\begin{gathered}
S^{p-1} \longrightarrow W(Z) / \partial W(Z) \stackrel{\Delta}{\longrightarrow} \\
\longrightarrow(W(Z) \times W(Z)) /(\partial W(Z) \times W(Z)) \stackrel{i d \times j(P, Q)}{\longrightarrow} \\
\longrightarrow(W(Z) \times \Omega M) /(\partial W(Z) \times \Omega M) \longrightarrow \\
\left.\stackrel{v}{\longrightarrow}\left(D^{q} \times Z(P, Q)\right) \times \Omega M /\left(S^{q-1} \times Z(P, Q)\right) \times \Omega M\right) \stackrel{p_{1} \times i d}{\longrightarrow} \\
\longrightarrow D^{q} \times \Omega M / S^{q-1} \times \Omega M=S^{q} \wedge \Omega M^{+}
\end{gathered}
$$

where the map $v$ is the identification of $W(Z)$ with $D^{q} \times Z(P, Q)$ provided by the framing $\iota^{+}$. Notice that $\partial W(Z)=S^{q-1} \times Z(P, Q)$ is transported by the flow $\gamma$ into $\partial A(Q) \subset A(Q)$ in such a way that $S^{q-1} \times\{x\} \subset \partial W(Z)$ is transported into $S^{q-1} \times\left\{x^{\prime}\right\} \subset S^{q-1} \times D^{n-q}=A(Q)$ by a homeomorphism. This implies that the compositions of the last two maps in (22) and the last three in (21) are homotopic. In turn, this shows that $\delta^{\prime \prime} \simeq h(P, Q)$ and concludes the proof of the theorem.

In the following we will consider Hopf complexes of certain particular pairs of spaces. The first basic example is given by index pairs $\left(N_{1}, N_{0}\right)$ of some isolated invariant set in a gradient flow on $M$. A cell decomposition of $N_{1}$ relative to $N_{0}$ produces as in Corollary 2.3 a complex with coefficients in $\pi_{*}^{S}\left(\Omega N_{1}^{+}\right)$. We fix the following convention: whenever talking about a Hopf complex of such an index pair we understand the complex obtained after 
changing coefficients via the inclusion map $N_{1} \rightarrow M$. Of course, this produces a complex with coefficients in $\pi_{*}^{S}\left(\Omega M^{+}\right)$. The second basic example of such pairs is given by the pair $\left(M^{\prime}, M\right)$ providing the global Conley index of some isolated invariant set (see \$1.1). Again, whenever talking about a Hopf complex of such a global Conley index we understand the complex with coefficients in $\pi_{*}^{S}\left(\Omega M^{+}\right)$obtained from a relative cell decomposition of $\left(M^{\prime}, M\right)$ with the coefficients changed by the canonical projection map $p: M^{\prime} \rightarrow M$ of the global index.

Corollary 2.13. The object $\left(C_{N}^{I}(f), d_{f}^{I}\right)$ associated to a local Morse function $(f, N)$ and introduced in Defintion 3 is indeed a chain complex and is identified with a Hopf complex of an index pair for $I_{N}(f)$.

Proof. Notice that in the setting of Definition 3 if $x \in \operatorname{Crit}_{a_{i}}^{N}(f)$ and $y \in$ Crit $a_{a_{i-1}}^{N}(f)$ for $I=\left(a_{1}, \ldots, a_{i-1}, a_{i}, \ldots\right)$, then $x$ and $y$ are consecutive critical points of $f$ in $N$. Use the theorem to identify the bordism classes of the connecting manifolds of type $Z(x, y)$ with the appropriate Hopf invariants and apply Corollary 2.3.

Remark 2.14. a. A different proof of $\left(d_{f}^{I}\right)^{2}=0$ follows by adapting to the non-compact case the method described in [5]. This is much more geometric in nature and is based on a precise description of the boundaries of the spaces of smooth flow lines joining two critical points that are not consecutive but might be joined by flow lines that are broken precisely once. One can also find there an explicit discussion of the signs in the formula $\left(d_{f}^{I}\right)^{2}=0$.

b. Clearly, Theorem 1 used in conjunction with Proposition 2.2 prove a bit more than the vanishing of the square of the differential of $C_{N}^{I}(f)$. Indeed, we obtain some relations among the Thom maps (of type $h(P, Q)$ ) associated to the connecting manifolds and not only among their stabilizations. It is harder to encode these relations in a Morse type complex the main problem being to find an appropriate coefficient ring (necessarily non-commutative). Moreover, even at the level of the bordism classes we get more than the relations needed to show that $C_{N}^{I}(f, \alpha)$ is a chain complex. Explicitely, if $P$ and $R$ are critical points that are connected by flow lines that are broken at most once, then we have $\sum_{Q \in C r i t_{*}(f)}+/-[Z(P, Q)][Z(Q, R]=0$ (the precise signs are in [5]; recall that $[Z(x, y)]$ is taken to be 0 whenever $x, y$ are not consecutive).

c. In view of the definition of the global Conley index $\bar{c}_{N}(f)=\bar{c}_{\gamma(f)}(I(f))$ we also obtain that $\left(C_{N}^{I}(f), d_{f}^{I}\right)$ is identified with a Hopf complex of the global index because a relative cell decomposition of an index pair for $I_{N}(f)$ produces also a relative cell decomposition of the global Conley index of this invariant set.

\subsection{Rigidity and duality for extended Morse complexes.}

In the first paragraph of this subsection we discuss certain continuation related properties of extended Morse complexes. The second paragraph 
is concerned with the behaviour of these complexes with respect to time reversion and stabilization.

\subsubsection{Continuation.}

Continuation induces a natural equivalence relation inside the space $\mathcal{F}_{l}(M)$ of pairs $(f, N)$ such that $f: M \rightarrow \mathbf{R}$ is a $C^{2}$-function and $N$ is an isolating neighbourhood for $\gamma(f)$ which, we recall, is the flow induced by the negative gradient of $f$. As before, we denote by $I_{N}(f)$ the maximal invariant set of this flow inside $N$. A pair $(f, N) \in \mathcal{F}_{l}(M)$ will be called an isolated local function on $M$.

Definition 5. The isolated local functions $\left(f_{0}, N_{0}\right),\left(f_{1}, N_{1}\right)$ are continuation equivalent if $I_{N_{0}}\left(f_{0}\right)$ and $I_{N_{1}}\left(f_{1}\right)$ viewed as isolated invariant sets in the flows $\gamma\left(f_{0}\right)$ and respectively $\gamma\left(f_{1}\right)$ are related by continuation (see $\S 1$.1. $)$.

Remark 2.15. a. Clearly, both the Conley index and the global Conley index are invariants with respect to continuation equivalence.

b. Given $(f, N) \in \mathcal{F}_{l}(M)$ there is a $C^{2}$-neighbourhood $\mathcal{U}$ of $f$ (in the weak topology) such that if $g \in \mathcal{U}$ then $(g, N)$ is also an isolated local function and is continuation equivalent to $(f, N)$.

Here is a useful consequence of the results in $\$ 2.1 .2$

Corollary 2.16. Assume that two local Morse functions $\left(f_{0}, N_{0}\right)$ and $\left(f_{1}, N_{1}\right)$ are continuation equivalent. There exists a morphism of graded abelian groups $m: \mathbf{Z}\left[\operatorname{Crit}_{*}^{N_{0}}\left(f_{0}\right)\right] \rightarrow \mathbf{Z}\left[\operatorname{Crit}_{*}^{N_{1}}\left(f_{1}\right)\right]$ wich extends to a chain-equivalence of free $\Omega_{*}^{f r}(\Omega M)$-modules

$$
m^{I}: C_{N_{0}}^{I}\left(f_{0}\right) \longrightarrow C_{N_{1}}^{I}\left(f_{1}\right)
$$

for each $I \subset \mathbf{N}$ for which both extended Morse complexes are defined.

Proof. Assume $C_{N_{0}}^{I}\left(f_{0}\right)$ and $C_{N_{1}}^{I}\left(f_{1}\right)$ are both defined. Because $\left(f_{0}, N_{0}\right)$ and $\left(f_{1}, N_{1}\right)$ are continuation equivalent then the global Conley indexes (see $\$ 1.1$ ) $\bar{c}_{N_{0}}\left(f_{0}\right)$ and $\bar{c}_{N_{1}}\left(f_{1}\right)$ coincide. The two extended Morse complexes above being identified by Corollary 2.13 to Hopf complexes of this same global index, the statement follows by applying Lemma 2.5.

Remark 2.17. There is also a purely geometric proof of a variant of this corollary which is based on the techniques that appear in [7]. The geometric method leads in fact to more powerful results because it may be applied in cases when the global Conley index is not defined. Moreover, it also shows that for any Morse function $(f, N)$ an arbitrary perturbation of the metric $\alpha$ inside $N$ does not modify the isomorphism type of the extended Morse complexes of $f$.

We will make use of the following notion of minimality.

Definition 6. A local Morse function $(f, N)$ is minimal if $C_{N}^{\mathbf{N}}(f)$ has trivial differential. In other words, the classical Morse complex of $\left.f\right|_{N}$ has trivial differential. 
If a local Morse function $(f, N)$ is minimal, then its number of critical points $\in N$ of index $i$ is equal to $r k\left(H_{i}\left(c_{N}(f) ; \mathbf{Z}\right)\right.$. Obviously, the integral homology of $c_{N}(f)$ is forced to be torsion free. The next statement immediately follows from Corollary 2.16.

Corollary 2.18. Assume that two local Morse functions $(f, N),\left(g, N^{\prime}\right)$ are minimal and continuation equivalent. Then for any index set $I$ we have $C_{N}^{I}(f) \approx C_{N^{\prime}}^{I}(g)$.

At this point we introduce a particular notation for certain special extended Morse complexes. Let $(f, N)$ be a local Morse function and let $I_{f}=\left\{k \in \mathbf{N}: \operatorname{Crit}_{k}^{N}(f) \neq \emptyset\right\}$. We denote by $\left(\mathcal{C}_{N}(f), d_{f}\right)$ the extended Morse complex $\left(C_{N}^{I_{f}}, d_{f}^{I}\right)$. This complex carries the most possible information in its differential among all extended Morse complexes of $(f, N)$. Recall the minimal Hopf complexes $\mathcal{C}_{\min }\left(X^{\prime}, X\right)$ that appear in Lemma 2.5 and are defined whenever $\left(X^{\prime}, X\right)$ admits a minimal cell-decomposition in the sense of that lemma.

From the results above we immediately obtain.

Corollary 2.19. Assume that the local Morse function $(f, N)$ is minimal. Then $\mathcal{C}_{\text {min }}\left(\bar{c}_{N}(f)\right)$ is defined and is isomorphic to $\mathcal{C}_{N}(f)$.

Remark 2.20. a. In practice, to apply the statement above one needs to construct a minimal cell decomposition for the global Conley index $\bar{c}_{N}(f)$ purely homotopically (this is possible, for example, if this Conley index has torsion free integral homology and is simply connected) and then compute $\mathcal{C}_{\min }\left(\bar{c}_{N}(f)\right)$ out of it.

b. One can, of course, use instead of the global Conley index an index pair $\left(N_{1}, N_{0}\right)$ for $I_{N}(f)$.

The existence of a minimal Morse function in a given continuation class can be insured only under circumstances somewhat restrictive. The result below follows, as shown in [23], from the classical techniques used by Smale in the proof of the h-cobordism theorem.

Proposition 2.21. 23] Assume the isolated local function $(f, N)$ has the following two properties:

(i) $c(f)$ has torsion free integral homology

(ii) there exists a bi-regular index block $\left(N_{1}, N_{0}, N_{2}\right)$ for $I_{N}(f)$ with $N_{i}$ simply connected for $i \in\{0,1,2\}$.

Then there is a minimal local Morse function continuation equivalent to $f$.

\subsubsection{Stabilization and duality of extended Morse complexes.}

For $(f, N) \in \mathcal{F}_{l}(M),\left(h, N^{\prime}\right) \in \mathcal{F}_{l}\left(M^{\prime}\right)$ we may define $f \oplus h: M \times M^{\prime} \rightarrow \mathbf{R}$ by $(f \oplus h)(x, y)=f(x)+h(y)$ and clearly, $\left(f \oplus h, N \times N^{\prime}\right) \in \mathcal{F}_{l}\left(M \times M^{\prime}\right)$ (where the metric on the product is the product metric). Moreover, by the basic properties of the Conley index we have $c_{N \times N^{\prime}}(f \oplus h)=c_{N}(f) \wedge c_{N^{\prime}}(h)$. Of particular iterest is the case when $h$ is a non-degenerate quadratic form 
$q: \mathbf{R}^{k} \rightarrow \mathbf{R}$ the metric on $\mathbf{R}^{k}$ is the euclidean one and $N^{\prime}$ is a disk $D^{k}$ containing 0. A function of the form $f \oplus q$ is called a stabilization of $f$ [9] [4][5]. Of course, in this case $c_{N^{\prime}}(q)=S^{\text {ind }(q)}$.

The behaviour of the extended Morse complexes with respect to stabilization is very easy to describe.

Corollary 2.22. Let $(f, N)$ be a local Morse function. Assume $q: \mathbf{R}^{k} \rightarrow \mathbf{R}$ is a non-degenerate quadratic form. For any index set $I \subset \mathbf{N}$ we have an isomorphism $\left.s^{\operatorname{ind}(q)}\left(\mathcal{C}_{N}^{I}(f), d_{f}^{I}\right) \approx \mathcal{C}_{N \times D^{k}}^{I^{\prime}}(f \oplus q),, d_{f \oplus q}^{I^{\prime}}\right)$ where $I^{\prime}=\{k+$ $\operatorname{ind}(q): k \in I\}$ and $s^{(-)}$is algebraic suspension.

Proof. The statement is immediate by noting that $f+q$ is Morse-Smale with respect to the sum metric $\alpha+$ euclidean on $N \times D^{k}$. Moreover, two consecutive critical points $P$ and $Q$ of $f$ remain consecutive for $f \oplus q$ and have the same connecting manifold. The framing of this connecting manifold also does not change stably and the map to the loop space $\Omega\left(M \times \mathbf{R}^{k}\right) \simeq \Omega M$ also remains the same up to homotopy.

It was shown in the first paper in this series [- 1 that if the stable normal bundle of the manifold $M$ (assumed there to be compact) is trivial, then for any flow $\gamma$ on $M$ and any isolated invariant set $S$ of $\gamma$ the Conley indexes of $S$ with respect to the direct flow $c_{\gamma}(S)$ and with respect to the inverse flow $c_{-\gamma}(S)$ are related by Spanier-Whitehead duality $\left(\right.$ here $\left.(-\gamma)_{t}(x)=\gamma_{-t}(x)\right)$. This duality relation was shown to be also valid, in this situation, for the homotopical data resulting from an attractor-repellor pair. In particular the connection maps of the attractor and the repellor with respect to the direct and inverse flows are Spanier-Whitehead duals. A partial, homotopical variant of this result in the Morse case was already present in [10]. A homological version in the case of general isolated invariant sets appeared in [16]. If the stable normal bundle of $M$ is no longer trivial, then it is natural to "estimate" the possible lack of duality. This was investigated in [4] by means of a certain Thom construction.

The last task of this sub-section is to use our understanding of connecting manifolds to explicit the deviation from Spanier-Whitehead duality (along the lines in [5]) for the case of the simplest type of attractor-repellor pair, consecutive non-degenerate critical points in a Morse-Smale gradient flow, and to then formulate the result globaly in terms of the extended Morse complexes.

On the purely homotopical side some duality results already appear in Corollary 2.11. Here we treat duality from the perspective of connecting manifolds and extended Morse complexes.

We return now to our manifold $M$ and we asssume further in this paragraph that there is a compactification $\bar{M}^{n}$ of $M$ which is included in a compact, closed manifold $M^{\prime}$ of dimension $n$. Let $\nu$ be the stable normal bundle of $M^{\prime}$ restricted to $M$. We will also denote by $\nu$ the resulting map $\nu: M \rightarrow B S O$. We will refer to this bundle $\nu$ as a stable normal bundle to $M$. 
There is an involution $*: \pi_{*}^{S}\left(\Omega M^{+}\right) \rightarrow \pi_{*}^{S}\left(\Omega M^{+}\right)$defined as follows: if $a \in \Omega_{k}^{f r}(\Omega M)$ is given by a map $a: S^{t} \rightarrow S^{t-k} \wedge \Omega M^{+}, t>>0$, then $a^{*}$ is represented by the composition $S^{t} \stackrel{a}{\rightarrow} S^{t-k} \wedge \Omega M^{+} \stackrel{i d \wedge \Delta}{\longrightarrow} S^{t-k} \wedge \Omega M^{+} \wedge$ $\Omega M^{+} \stackrel{i d \wedge \Omega \nu \wedge i d}{\longrightarrow} S^{t-k} \wedge \mathbf{S O}^{+} \wedge \Omega M^{+} \stackrel{\mu \wedge i d}{\longrightarrow} S^{t-k} \wedge \Omega M^{+} \stackrel{i d \wedge-1}{\longrightarrow} S^{t-k} \wedge \Omega M^{+}$ where $\mu$ is induced by the action of $\mathbf{S O}(t-k+1)$ on $S^{t-k},-1$ is the map reversing the orientation of the loops in $\Omega M$ and $\Delta$ is the diagonal.

Assume that $(\mathcal{C}, d)$ is an $\pi_{*}^{S}\left(\Omega M^{+}\right)$-chain complex. We define its $n$-dual $\left(\mathcal{C}^{n}, d^{*}\right)$ by an isomorphism $*: \mathcal{C}_{n-k}^{*} \approx \mathcal{C}_{k}$ and $d^{*}\left(x^{*}\right)=\sum d^{*}\left(x^{*}, y^{*}\right) y^{*}$ such that $d^{*}\left(x^{*}, y^{*}\right)=(d(y, x))^{*}$ with $d(y)=\sum d(y, x) x$.

Corollary 2.23. Assume $(f, N)$ is a local Morse function on $M$.

(i) Suppose that $P, Q$ are consecutive critical points of $\left.f\right|_{N}$. If $[Z(Q, P)] \in$ $\Omega_{*}^{f r}(\Omega M)$ is the bordism class of the connecting manifold of $Q$ and $P$ viewed as consecutive critical points for the function $-f$, then $[Z(Q, P)]=$ $[Z(P, Q)]^{*}$.

(ii) we have an isomorphism $\left(\mathcal{C}_{N}(-f), d_{-f}\right) \approx\left(\mathcal{C}_{N}(f)^{*},\left(d_{f}\right)^{*}\right)$.

(iii) if $f$ is a minimal Morse function and $M$ is closed, $N=M$, then we have $\mathcal{C}_{N}(f) \approx\left(\mathcal{C}_{N}(f)\right)^{*}$ the isomorphism being induced by the Poincaré duality isomorphism.

Proof. The first point is an easy consequence of the fact that, as a toplogical space, the connecting manifold of $P$ and $Q$ viewed as consecutive critical points for $f$ is the same as that of $Q$ and $P$ as consecutive critical points for $-f$. Moreover, the inclusion into $\Omega M$ changes by the reversion of the loops. It remains to compare the framings of $Z(P, Q)$ in $S_{f}^{u}(P)$ and $S_{f}^{s}(Q)$ (the subscript $f$ indicates that the flow used is $\gamma(f))$. Let $E\left(M^{\prime}\right)$ be a tubular neighbourhood of $M^{\prime}$ inside a high dimensional sphere. Of course, $E\left(M^{\prime}\right)$ is diffeomorphic to the total space of the disk bundle associated to $\nu$. Let $q: E\left(M^{\prime}\right) \rightarrow \mathbf{R}$ be a function measuring the square of the distance from $M^{\prime}$ inside $E\left(M^{\prime}\right)$. Inside each fibre of the disk bundle $E\left(M^{\prime}\right)$ the function $q$ is a positive definite quadratic form. Let $E(M)$ be the restriction of the bundle to $M$ and consider now the function $\bar{f}: E(M) \rightarrow \mathbf{R}, \bar{f}(z)=f(p(z))+q(z)$ where $p: E\left(M^{\prime}\right) \rightarrow M^{\prime}$ is the projection of the bundle. It is easy to see that $\bar{f}$ is a local Morse function after choosing an appropriate metric on $E(M)$. Assume that the rank of $E(M)$ is $k$. Notice that $S_{\frac{u}{f}}(P)=S_{f}^{u}(P)$ and $S \frac{s}{f}(Q)=\Sigma^{k} S_{f}^{s}(Q)$. Because a sphere has a trivial normal bundle the framings of $Z(P, Q)$ in $S \frac{u}{f}(P)$ and $S \frac{s}{f}(Q)$ are stably the same up to sign (by the more general results in [1] or those in [10]). On the other hand the framings of $Z(P, Q)$ in $S_{f}^{u}(P)$ and $S_{\frac{u}{f}}(P)$ are the same. However, the framings of $Z(P, Q)$ in $S_{f}^{s}(Q)$ and $S_{\frac{s}{f}}(Q)$ differ by the twisting coming from the normal bundle of $M^{\prime}$ in $E\left(M^{\prime}\right)$ which is $\nu$. This immediately implies (i). The second point is an obvious consequence of the first. The third is implied by the fact that both $f$ and $-f$ are minimal and as $M$ is compact they are in the same continuation class. 
Remark 2.24. Notice that, in the smooth context, this corollary is an extension of Corollary 2.11. It can be used to detect homotopy equivalences that do not admit diffeomorphisms as representatives. The third point can be interpreted as saying that the Poincaré duality isomorphism respects the structure contained in the minimal Hopf complex of $M$.

\section{BOUNDED, CLOSED AND PERIODIC ORBITS FOR HAMILTONIAN FLOWS.}

The setting is as follows. We assume from now on that our fixed manifold $M$ admits a symplectic form $\omega$ that we fix. Assume that $f: M \rightarrow \mathbf{R}$ is a smooth function. The hamiltonian vector field induced by $f, H_{f}$, is defined by requiring the equation $\omega\left(H_{f}, Y\right)=-d f(Y)$ to hold for all vector fields $Y$ on $M$. The flow induced by the vector field $H_{f}$ will be denoted by $h^{f}$ and is called the hamiltonian flow of $f$.

In searching for bounded and periodic orbits the following immediate remark is useful. For any riemannian metric $\alpha$ on $M$ we have $\alpha\left(-\nabla^{\alpha}(f), H_{f}\right)=$ $-d f\left(H_{f}\right)=\omega\left(H_{f}, H_{f}\right)=0$ thus $H_{f}$ and $\nabla^{\alpha}(f)$ are $\alpha$-orthogonal and each hypersurface $f^{-1}(y)$ is $h^{f}$-invariant. Moreover, it is well known that the problem of existence of periodic orbits of $h^{f}$ on some regular hypersurface $A=f^{-1}(a)$ does only depend on $A$ and not on $f$. This is why one can expect that some topological constraints on $f$ can impose enough tension on the hypersurfaces $f^{-1}(a)$ as to force some recurrence phenomena.

The case that has been extensively studied in the literature corresponds to a function $f$ that has some compact regular hypersurfaces. The simplest way to insure this condition is to take $f$ to be a function that attains an extremum on a compact set. Suppose $A=f^{-1}(a)$ is a regular, compact hypersurface. Then, of course, every orbit of $h^{f}$ originating in $A$ is bounded, Poincaré's recurrence theorem shows that, except for a set of zero measure, all points are recurrent. As for the existence of periodic orbits there are two complementary approaches. The first, based on the $C^{1}$ closing lemma of Pugh and Robinson, shows that for a generic choice of $f$ the recurrent points can be transformed into periodic ones and thus, in this compact case, "generically", periodic orbits are abundant. The second approach has been pursued succesfully by Rabinowitz [22], Weinstein [29] as well as Ekeland, Floer, Hofer, Moser, Zehnder and many other authors (see [12] and [11] for surveys on the subject). It consists in using methods adapted to the specific form of a fixed, particular $f$ and namely variational methods on the space of the free loops in $M$ to deduce the existence of periodic orbits for this fixed $f$.

In the following we focus on the non-compact context. We assume therefore that $M$ is non-compact and, as indicated in the introduction, at the heart of our results are certain criteria that will insure the existence of bounded orbits. In the first sub-section it is the lack of (Spanier-Whitehead) duality which leads to such existence results. In the second sub-section we 
use the non-vanishing of the differential in an extended Morse complex, or (given the results in Section 2) equivalentely, the presence of a non-vanishing relative Hopf invariant. It should be pointed out that the key advantage of the second method is that the bounded orbits that are produced in this way are better localized, in particular, they belong to regular hypersurfaces, fact that can not be guaranteed by the lack-of-duality approach. We then use the $C^{1}$-closing lemma to obtain generically periodic or, (in the case of the lack of Spanier-Whitehead duality method) closed (possibly homoclinic) orbits. Compared to the understanding of the compact case our results only correspond to the first, "generic", point of view. By following the arguments below it is easy to see that, in fact, both methods used to produce bounded orbits actually apply not only to hamiltonian flows but to any flows that are orthogonal to gradient ones. The third sub-section contains some explicit examples as well as the interpretation of our various results in terms of the invariant $d(-)$ mentioned in the introduction.

It is to be expected that by adding some appropriate analytical assumptions to our homotopical conditions one will obtain non-generic existence results. For example, I do not know of any examples of contact hypersurfaces that carry bounded characteristics and do not carry closed ones.

For now, let us notice that the bounded orbit existence problem is nontrivial.

Example 1. Let $M=\mathbf{R}^{2 k}$ be endowed with the symplectic form $\omega_{0}=$ $\sum_{i=1}^{k} d x_{i} \wedge d y_{i}$ and let $f\left(x_{1}, y_{1}, \ldots, x_{k}, y_{k}\right)=\sum_{i=1}^{k} f_{i}\left(x_{i}, y_{i}\right)$ with $f_{i}: \mathbf{R}^{2} \rightarrow$ $\mathbf{R}$ a smooth, analytic function with a single singularity at $0 \in \mathbf{R}^{2}$ which is different from an extremum, $1 \leq i \leq k$. Then $h^{f}$ has no bounded, nontrivial orbits. Moreover, the family of functions $f^{\prime}: \mathbf{R}^{2 k} \rightarrow \mathbf{R}$ such that $h^{f^{\prime}}$ has some non-trivial bounded orbit is not dense in any $C^{2}$-(weak) neighbourhood of $f$. It is useful to remark at this point that, if 0 is a totally degenerate critical point of $f$, then arbitrarily close to $f$ ( in the strong $C^{2}$ topology) one can find functions $g$ that have some compact level surfaces (one can construct such a function by just forcing the appearance of a local maximum close to the origin in $\mathbf{R}^{2 k}$ ) and thus the hamiltonians of type $h^{g}$ will have bounded non-trivial orbits. It is easy to see that in the example above, under the additional assumption that 0 is a non-degenerate critical point, the linearized hamlitonian flow at 0 is defined and it does not have any (non-trivial) periodic orbits. However, there are more sophisticated examples (the most famous produced by Moser [17) where no bounded orbits of the hamiltonian flow exist even if the linearized flow at the singular point has plenty of periodic ones.

\subsection{Lack of self - duality and closed orbits.}

Recall that for $(f, N) \in \mathcal{F}_{l}(M)$ we denote by $c_{N}(f)$ the Conley index of the maximal invariant set $I_{N}(f)$ of $f ; \bar{c}_{N}(f)$ is the global Conley index (both with respect to the negative gradient of $f$ ). 
We start with a result that continues an idea that has first been exploited in [6]. Recall from sub-section 2.3.2 that a stable normal bundle of $M$ is the stable normal bundle of a closed manifold $M^{\prime}$ of same dimension as $M$ and which contains a compactification of $M$. Using this normal bundle recall also that we can construct the dual of an extended Morse complex .

Proposition 3.1. Assume that a normal bundle $\nu$ to $M$ is defined. Suppose that $(f, N) \in \mathcal{F}_{l}(M)$ is fixed and suppose that it has the property that $I_{N}(f) \subset f^{-1}(v)$ for some isolated critical value $v \in \mathbf{R}$ of $f$. Suppose that one of the following conditions is satisfied:

(i) the restriction of $\nu$ to an isolating neighbourhood of $I(f)$ is trivial and $c_{N}(f)$ is not Spanier-Whitehead self n-dual.

(ii) $f$ is continuation equivalent to a minimal Morse function, and $\mathcal{C}_{\min }\left(\bar{c}_{N}(f)\right)$ is not isomorphic to $\left(\mathcal{C}_{\min }\left(\bar{c}_{N}(f)\right)^{*}\right.$.

Then the hamiltonian flow $h^{f}$ has the property that for any isolating neighbourhood $K$ of $I_{N}(f)$ there is a bounded orbit of $h^{f}$ that is included in $K$ and intersects the boundary of $K$.

Proof. We first claim that each of the conditions at (i) and (ii) implies that the invariant sets $I_{N}(f)$ and, respectively, $I_{N}(-f)$ are not related by continuation. Indeed, if they would be related by continuation, then $c_{N}(f)=$ $c_{N}(-f)$. But as $\nu$ is trivial on an isolating neighbourhood of the maximal invariant set of $f$, by results in [4], we have that $c_{N}(-f)$ is an $n$ - SpanierWhitehead dual of $c_{N}(f)$ hence, our claim follows from point (i) (we recall that the spaces $A, B$ with the homotopy type of finite $C W$-complexes are $n$ Spanier-Whitehead duals if there exist $A^{\prime}, B^{\prime}$ complementary in an $S^{2 m+n+1}$ sphere and with $A^{\prime} \simeq \Sigma^{m} A, B^{\prime} \simeq \Sigma^{m} B$ ). Similarly, condition (ii) also implies the non-existence of this continuation by applying Corollary 2.23 to the minimal Morse function continuation equivalent to $f$ and equating its minimal extended Morse complex with $\mathcal{C}_{\min }\left(\bar{c}_{N}(f)\right.$ ) (by Corollary 2.19). This Hopf complex only depends on the global Conley index of $f$. We then notice that if $I_{N}(f)$ and $I_{N}(-f)$ are related by continuation, then their global Conley indexes are the same. Applying the same argument to the negative of the minimal Morse function in the continuation class of $f$ we obtain our claim.

We now arrive to the key idea of the proof. We notice that if the conclusion of the proposition would be false, then there would exist a continuation between $I_{N}(f)$ and $I_{N}(-f)$. Indeed, we let $u, v, w:[-1,1] \rightarrow[0,1]$ be a smooth partition of the unity such that $u^{-1}(0)=[0,1], v^{-1}(0)=[-1,0], w^{-1}(0)=$ $[1,-1 / 2] \cup[1 / 2,1] u([-1,-1 / 2])=1, v([1 / 2,1])=1, w([-1 / 4,1 / 4])=1$, $u^{\prime}(t)<0$ for $t \in(-1 / 2,0), v^{\prime}(t)>0$ for $t \in(0,1 / 2), w^{\prime}(t) \operatorname{sign}(t)<0$ for $t \in(-1 / 2,-1 / 4) \cup(1 / 4,1 / 2)$. We now define a smooth one parameter family of vector fields on $M, X_{\tau}=-u(\tau) \nabla(f)+w(\tau) H_{f}+v(\tau) \nabla(f)$. Suppose that $K$ is an isolating neighbourhood of $I_{N}(f)$ which does not satisfy the conclusion of the proposition. Then $K \times[-1,1]$ is an isolating neighbourhood for the flow $\Gamma$ on $M \times[-1,1]$ given by letting $\Gamma_{\tau}=\left.\Gamma\right|_{M \times\{\tau\}}$ be induced by 
$X_{\tau}$. This happens because for all values of $\tau \neq 0$ one of the functions $f$ or $-f$ is a Lyapounov function for the flow $X_{\tau}$ and as $I_{N}(f)$ is contained in a single level hypersurface of $f$ it results that the maximal compact invariant set for $\Gamma_{\tau}$ inside $K \times\{\tau\}$ is $I_{N}(f)$. For $\tau=0$ our assumption that all orbits of $h^{f}$ that are contained in $K$ do not intersect $\partial K$ implies that $K \times\{0\}$ is an isolating neighbourhood for $\Gamma_{0}$. This means that $I_{N}(f)$ can be continued to $I_{N}(-f)$ and leads to a contradiction.

By a closed orbit in a flow we mean a periodic orbit or the trajectory of some point $x$ whose $\omega$-limits $\omega(x)$ and $\omega^{*}(x)$ both reduce to the same single point (this last case is refered to as a homoclinic closed orbit).

Corollary 3.2. Let $(f, N) \in \mathcal{F}_{l}(M)$. There is a family of functions $\mathcal{F}$ dense in a $C^{2}$ - neighbourhood of $f$ in the $C^{2}$-strong topology such that for each $f^{\prime} \in \mathcal{F}$ the hamiltonian flow $h^{f^{\prime}}$ has at least

$$
\sum_{j \neq n / 2} r k\left(H_{j}(c(a) ; \mathbf{Z})\right.
$$

possibly homoclinic, non-trivial closed orbits.

Proof. We fix a sufficientely small $C^{2}$-neighbourhood $\mathcal{U}$ of $f$ such that any function $g \in \mathcal{U}$ has the property that $N$ is an isolating neighbourhood for $\gamma(g)$. There is dense family $\mathcal{F}^{\prime} \subset \mathcal{U}$ such that $\mathcal{F}^{\prime}$ is dense in $\mathcal{U}$, each critical level of a function $f^{\prime} \in \mathcal{F}^{\prime}$ only contains a single critical point inside $N$, $\left(f^{\prime}, N\right)$ is a local Morse function and is continuation equivalent to $f$ (this is true because starting with any isolated local function $(g, N)$ we may perform a compactly supported, arbitrarily small modification to obtain a function $f^{\prime}$ as above). Let $f^{\prime} \in \mathcal{F}^{\prime}$. An immediate application of the Morse inequalities shows that such a function has at least $r k\left(H_{k}\left(c_{N}(f) ; \mathbf{Z}\right)\right)$ critical points of index $k$. We may apply Proposition 3.1 for each critical point $P$ of $f^{\prime}$ such that $\operatorname{ind}(P) \neq n / 2$. We obtain that for each sufficiently small compact set $K_{P}$ around $P$ the hamiltonian flow $h^{f^{\prime}}$ has at least one non-trivial bounded orbit in $K_{P}$ that intersects non-trivially the boundary of $K_{P}$. This means that either this orbit has both its $\omega$ and $\omega^{*}$ limits equal to $P$ - in which case it is a homoclinic orbit - or one of these limits belongs to $\Omega_{c}\left(h^{f^{\prime}}\right) \cap K_{P}-\{P\}$ where for a flow $\gamma, \Omega_{c}(\gamma)$ is the set of the non-wandering points of $\gamma$ that have a non-vanishing $\omega$ or $\omega^{*}$ limit. In this case, let $y \in \Omega_{c}\left(h^{f^{\prime}}\right) \cap K_{P}-\{P\}$. By the $C^{1}$ closing lemma of Pugh and Robinson [21] (in particular the argument in $\S 11$ ) there is a $C^{1}$-deformation of $H_{f^{\prime}}$ that has a compact support (only depending on the orbit of $y$ ), that can be assumed arbitrarily small and whose result is a hamiltonian vector field $H_{f^{\prime \prime}}$ whose associated flow has a periodic orbit that is close to $y$ and therefore is non-constant. Because the deformation leading to $H_{f^{\prime \prime}}$ is compactly supported it results that we may obtain $f^{\prime \prime}$ as $C^{2}$-close to $f^{\prime}$ as desired and by apply this construction for each such critical point $P$. This proves the existence of the family $\mathcal{F}$. 


\subsection{Non-vanishing of Hopf invariants and periodic orbits.}

In this sub-section we search for bounded and periodic orbits that are situated on regular level surfaces.

Let $(f, N)$ be a local Morse function such that $I_{N}(f)$ satisfies the property $(*)$ from the introduction. Before stating the main theorem of this section we indicate the role of property $(*)$ in this context. By definition, this property provides an index pair $\left(N_{1}, N_{0}\right)$ of $I_{N}(f)$ with $N_{0} \subset f^{-1}(a)$ with $a$ a regular value of $\left.f\right|_{N_{1}}$. By using an appropriate Lyapounov function we see that we may immediately assume that there is a strong index block $\left(N_{1}, N_{0}, N_{2}\right)$ with $N_{0} \subset f^{-1}(a)$ that we fix. For each $P \in \operatorname{Crit}^{N}(f)$ we see that $W_{N}^{u}(P) \cap \partial N_{1}=W_{N}^{u}(P) \cap \partial f^{-1}(a)$. We will discuss criteria insuring that property $(*)$ is satisfied in $\delta 3.3$.

Let $P, Q \in \operatorname{Crit}^{N}(f)$ such that their indexes $p, q$ are succesive in $\{k$ : $\left.\operatorname{Crit}_{k}^{N}(f) \neq \emptyset\right\}$. As in the setting of Theorem 1 1 we consider the relative Hopf invariant $H\left(j_{P}, j_{Q}\right)$. Recall that we denote by $\left[H\left(j_{P}, j_{Q}\right)\right] \in \pi_{*}^{S}\left(\Omega M^{+}\right)$ the stable class of $H\left(j_{P}, j_{Q}\right)$.

Theorem 2. With the assumptions above, if $\left[H\left(j_{P}, j_{Q}\right)\right] \neq 0$, then arbitrarily close to $f$ in the strong $C^{2}$ topology there is a function $\bar{f}$ such that there are infinitely many regular values $v$ of $\bar{f}, f(Q)<v<f(P)$ with the property that $\bar{f}^{-1}(v) \cap N_{1}$ contains a periodic orbit of $h^{\bar{f}}$.

Remark 3.3. Because $\left.f\right|_{N}$ is Morse, any function $f^{\prime}$ sufficiently $C^{2}$-close to $f$ is also Morse after restriction to $N$ and has the same type and number of critical points as $f$ there.

The proof of the theorem occupies the rest of this subsection

Assume the setting of Theorem 2. The main step is again to detect bounded orbits and consists in proving the next statement. In its turn, this has considerable intrinseque interest as it applies to the function $f$ itself (whithout requiring any perturbation). Moreover, there exist examples when $\left[H\left(j_{P}, j_{Q}\right)\right]=0$ but $H\left(j_{P}, j_{Q}\right) \neq 0$.

Proposition 3.4. Assume that one of the following two conditions is satisfied

(i) $H\left(j_{P}, j_{Q}\right) \neq 0$ and there are no critical points $R \in \operatorname{Crit}_{q}^{N}(f)$ with $f(Q)<f(R)<f(P), Z(P, R) \neq \emptyset$.

(ii) $\left[H\left(j_{P}, j_{Q}\right] \neq 0\right.$ and each $R \in \operatorname{Crit}_{q}^{N}(f)$ with $f(Q)<f(R)<f(P)$ verifies $\left[H\left(j_{P}, j_{R}\right)\right]=0$.

then there exists $\delta>0$ such that for all $v \in(f(Q), f(Q)+\delta)$ the intersection $V_{v}=f^{-1}(v) \cap \operatorname{Int}\left(N_{1}\right)$ is regular and contains at least one bounded orbit of the hamiltonian flow $h^{f}$.

Proof. We start with a general simple, auxiliary result. 
Lemma 3.5. Assume $\gamma: M^{\prime} \times \mathbf{R} \longrightarrow M^{\prime}$ is a flow that has an isolated invariant set $S \subset M$. Assume $K \subset M^{\prime}$ is an isolating neighbourhood of $S$. Let $K^{\prime}$ be a compact set such that $K \subset \operatorname{Int}\left(K^{\prime}\right)$ and $I_{\gamma}\left(K^{\prime}\right)=S$. There is an index pair $\left(L_{1}, L_{0}\right)$ for $S$ such that $K \subset \operatorname{Int}\left(L_{1}-L_{0}\right)$ and $L_{1} \subset K^{\prime}$.

Proof. This is a simple variation on the construction of index pairs in 24. For a compact set $T \subset K^{\prime}$ with $S \subset \operatorname{Int}(T)$ let $W_{T}=\left\{x \in K^{\prime}: \exists t \in\right.$ $\left.\mathbf{R}^{+}, \gamma_{[0, t]}(x) \subset K^{\prime}, \gamma_{t}(x) \in T\right\}$. This set is compact. Let $K^{\prime \prime} \subset \operatorname{Int}\left(K^{\prime}\right)$ be a second compact set with $K \subset \operatorname{Int}\left(K^{\prime \prime}\right)$ and let $U \subset W_{K^{\prime \prime}}$ be an open neighbourhood of $W_{K}$. Let $L_{0}=\left\{x \in K^{\prime}: \exists t \in \mathbf{R}^{+}, \gamma_{[-t, 0]}(x) \subset K^{\prime}, \gamma_{-t}(x) \in\right.$ $\left.K^{\prime}-U\right\}$. This set is positively invariant in $K^{\prime}$, compact and $K \cap L_{0}=\emptyset$. Let $L_{1}^{\prime}=\left\{x \in K^{\prime}: \exists t \in \mathbf{R}^{+}, \gamma_{[-t, 0]}(x) \subset K^{\prime}, \gamma_{-t}(x) \in K^{\prime \prime}\right\}$. This is again compact and we take $L_{1}=L_{0} \cup L_{1}^{\prime}$. It is easy to verify that $\left(L_{1}, L_{0}\right)$ is an index pair.

We return now to the proof of Proposition 3.4.

To simplify notation we let $W^{u}(-)=W_{N_{1}}^{u}(-)$ and similarly for the stable manifolds. In the same way we drop the index $N_{1}$ (or $N$ ) in the notation for the various isolated invariant sets as long as everything takes place in the isolating neighbourhood $N_{1}$. Moreover, because all our arguments will take place in $N_{1}$ we may assume (after possibly modifying the vector field $H_{f}$ away from $N_{1}$ ) that $h^{f}$ is a flow on $M$ (and not only a partially defined one).

The first step is to assume that we are in a particular situation.

Lemma 3.6. If $H\left(j_{P}, j_{Q}\right) \neq 0$ and all values $w \in(f(Q), f(P))$ are regular for $\left.f\right|_{\operatorname{Int}\left(N_{1}\right)}$, then for some $\delta^{\prime}>0$ and each $v \in\left(f(Q), f(Q)+\delta^{\prime}\right)$ the regular hypersurface $V_{v}$ contains at least one bounded orbit of the hamiltonian flow $h^{f}$.

Proof. For a value $v \in(f(Q), f(P))$, recall $V_{v}=f^{-1}(v) \cap \operatorname{Int}\left(N_{1}\right)$ be the corresponding level hypersurface of $f$. The condition $(*)$ implies that flow lines of $\gamma=\gamma(f)$ originating in $P$ intersect $V_{v}$. We take $\delta^{\prime}$ sufficiently small such that for $v \in\left(f(Q), f(Q)+\delta^{\prime}\right)$ all the flow lines arriving in $Q$ also have to cross $V_{v}$. We now fix such a $v \in\left(f(Q), f(Q)+\delta^{\prime}\right)$ and let $V=V_{v}$. Let $S(P)=S^{u}(P)=W^{u}(P) \cap V$ be the unstable sphere of $P$ and $S(Q)=S^{s}(Q)=W^{s}(Q) \cap V_{v}$ be the stable sphere of $Q$. As there are no critical values in the interval $(f(Q), f(P))$ we obtain that $S(P) \approx S^{p-1}$ and $S(Q) \approx S^{n-q-1}$. Let $K^{\prime} \subset V$ be a compact neighbourhood of the union $S(P) \cup S(Q)$.

We intend to show by contradiction that $K^{\prime}$ contains (at least) one orbit of $h^{f}$. We now suppose that this is not the case and therefore the maximal compact invariant set of $h^{f}$ inside $K^{\prime}, I_{h^{f}}\left(K^{\prime}\right)$, is void. Let $Z=Z(P, Q)=$ $S(P) \cap S(Q)$. Recall that, as in Theorem 1, we have the framed embedding $i: Z \subset S(P)$ and the map $j=j(P, Q): Z(P, Q) \rightarrow \Omega M$. Our purpose is to see that the assumption that $I_{h^{f}}\left(K^{\prime}\right)=\emptyset$ is sufficient to construct a nullbordism of the the pair $(i, j)$ inside $S^{p-1} \times[0,1]$. This immediately leads to 
a contradiction. Indeed, if the couple $(i, j)$ is bordant to 0 inside $S^{p-1} \times[0,1]$ then the Thom map associated to $(i, j), t=h(P, Q): S^{p-1} \rightarrow S^{q} \wedge\left(\Omega M^{+}\right)$, is null-homotopic. But Theorem 1 claims that $h(P, Q)$ is homotopic (up to sign) to $H\left(j_{P}, j_{Q}\right)$ which is non-trivial by hypothesis.

We notice that $K^{\prime}$ is an isolating neighbourhood and as $S(P) \cup S(Q)$ is compact we may find (by Lemma 3.5) an index pair $\left(L_{1}, L_{0}\right)$ of $I_{h^{f}}\left(K^{\prime}\right)$ such that $S(P) \cup S(Q) \subset \operatorname{Int}\left(L_{1}-L_{0}\right), L_{1} \subset K^{\prime}$ (the fact that $I_{h^{f}}\left(K^{\prime}\right)=\emptyset$ does not prevent the application of that lemma). Moreover, by the construction of regular index pairs in [24] we may even assume that $\left(L_{1}, L_{0}\right)$ is a regular index pair. Indeed, we may construct a $C^{1}$ Lyapounov function $l: L_{1} \rightarrow$ $[0,1]$ for the flow $h^{f}$ with the additional property that it vanishes precisely on $L_{0}$. Now, there is some $\tau>0$ such that $l(x)>2 \tau$ if $x \in S(P) \cup S(Q)$. It now suffices to replace $L_{0}$ with $l^{-1}([0, \tau])$ and the pair $\left(L_{1}, L_{0}\right)$ becomes a regular index pair. The fact that $I_{h f}\left(K^{\prime}\right)=\emptyset$ implies that for each $x \in L_{1}-L_{0}$ the value $t_{x}=\sup \left\{t \in[0, \infty): h_{[0, t]}^{f}(x) \subset L_{1}-L_{0}\right\}$ is well-defined and finite. Clearly, $t_{x}$ is the "arrival time" of $x$ in $L_{0}$. Because the index pair $\left(L_{1}, L_{0}\right)$ is regular the arrival time function $T: L_{1} \rightarrow[0, \infty)$ defined by $T(x)=t_{x}$ if $x \in L_{1}-L_{0}$ and $T(x)=0$ otherwise, is continuous. As $L_{1}$ is compact the function $T$ attains its maximum which, to simplify notation, can be assumed to be equal to 1 .

Let $\psi: L_{1} \times[0,1] \rightarrow L_{1}$ be the function $\psi(x, t)=h_{\min \{t, T(x)\}}^{f}(x)$. This function is clearly continuous and, moreover, at a point $(x, t)$ such that $t<$ $T(x)$ it has the same order of differentiability as $h^{f}$. Let $\psi^{\prime}: S(Q) \times[0,1] \rightarrow$ $L_{1} \times[0,1]$ be given by $\psi^{\prime}(x, t)=(\psi(x, t), t)$. We denote by $W$ the image of $\psi^{\prime}$ and we let $N^{\prime}=\operatorname{Int}\left(L_{1}-L_{0}\right) \times[0,1]$ and $W^{\prime}=W \cap N^{\prime}$. We remark that $W^{\prime}$ is a $C^{1}$ closed submanifold in $N^{\prime}$. On the other hand let $u: S(P) \rightarrow L_{1}$ be the inclusion. We consider the map $\phi: S(P) \times[0,1] \rightarrow N^{\prime} \times[0,1], \phi(x, t)=$ $(u(x), t)$. This map is $C^{1}$ and an embedding. By standard transversality theory we may find another $C^{1}$ embedding, $\phi^{\prime}: S(P) \times[0,1] \rightarrow L_{1} \times[0,1]$ which is arbitrarily close to $\phi$, is transverse to $W^{\prime}$ and coincides with $\phi$ when restricted to $S(P) \times\{0\}$. Let $S^{\prime}$ be the image of $\phi^{\prime}$ and notice that because $h_{T(x)}^{f}(x) \in L_{0}$ we obtain that $S^{\prime} \cap W^{\prime} \cap\left(N^{\prime} \times\{1\}\right)=\emptyset$. If we let $C=\left(\phi^{\prime}\right)^{-1}\left(W^{\prime}\right)$ this implies that $C$ is a $C^{1}$ submanifold of $S(P) \times[0,1]$ such that $\partial C=C \cap(S(P) \times\{0\})=Z \times\{0\}$.

To see that $C$ does indeed provide the wanted null-bordism we still need to show that the structure given by $(i, j)$ can be extended over $C$. We start with the map $j$ whose definition we recall. Let $w$ be an oriented path in $M$ that joins, in order, the critical point $Q$ to the critical point $P$. For each point $y \in Z$ there is a unique flow line, $w(y)$, of the negative gradient flow $\gamma(f)$ of $f$ containing $y$ and joining $P$ to $Q$. The map $j$ is defined by $j(y)=w(y) * w$ where $*$ is concatenation of paths (as $M$ is simply-connected the homotopy type of this map does not depend on $w$ ). Notice that for each point $z \in S(P)$ there is a unique flow line $w^{\prime}(z)$ of $\gamma(f)$ originating in $P$ and ending in $z$. Similarly, for each $z \in S(Q)$ there 
is a unique flow line $w^{\prime \prime}(z)$ of $\gamma(f)$ originating in $z$ and ending in $Q$. If $y \in Z$, then $w(y)=w^{\prime}(y) * w^{\prime \prime}(y)$. Take now some point $(z, t) \in W^{\prime}$. Then we have $(z, t)=\left(h_{t}^{f}(x(z, t)), t\right)$ for a unique $x(z, t) \in S(Q)$. Let $w^{\prime \prime}(z, t)=\left\{x \in M: \exists v^{\prime}, f(Q) \leq v^{\prime} \leq v, x=h_{t}^{f}\left(w^{\prime \prime}(x(z, t)) \cap f^{-1}\left(v^{\prime}\right)\right\}\right.$. It is easy to see that $w^{\prime \prime}(z, t)$ is a continuous path joining $(z, t)$ to $Q \times\{t\}$ and, of course, $w^{\prime \prime}(z, 0)=w^{\prime \prime}(z) \times\{0\}$. Consider now a point $z^{\prime} \in S^{\prime}$. Then $z^{\prime}=\phi^{\prime}\left(x^{\prime}\left(z^{\prime}, t\right), t\right)$ for a unique couple $\left(x^{\prime}\left(z^{\prime}, t\right), t\right) \in S(P) \times[0,1]$. We may assume $\phi^{\prime}$ close enough to the constant embedding $\phi$ such that for all $z^{\prime} \in S^{\prime}$ there is a unique minimal geodesic in $V$ that joins $\left(x^{\prime}\left(z^{\prime}, t\right), t\right)$ to $z^{\prime}$. We denote the path given by this geodesic and ending in $z^{\prime}$ by $w^{\prime \prime \prime}\left(z^{\prime}\right)$. Let $w^{\prime}\left(z^{\prime}\right)=\left(w^{\prime}\left(x^{\prime}\left(z^{\prime}, t\right)\right) \times\{t\}\right) * w^{\prime \prime \prime}\left(z^{\prime}\right)$. This path starts in $P \times\{t\}$ and ends in $z^{\prime} \in S^{\prime}$. If $(x, 0) \in S(P) \times\{0\}$ then $\left.w^{\prime}(x, 0)\right)$ coincides with $w^{\prime}(x) \times\{0\}$.

We define $J^{\prime}: C \rightarrow C^{0}\left(S^{1}, M \times[0,1]\right)$ by $J(z)=w^{\prime}\left(\phi^{\prime}(z)\right) * w^{\prime \prime}\left(\phi^{\prime}(z)\right) *$ $w \times\{t\}$. It is very easy to check that this map is continuous. The projection $M \times[0,1] \rightarrow M$ induces a coninuous map $e: C^{0}\left(S^{1}, M \times[0,1]\right) \rightarrow C^{0}\left(S^{1}, M\right)$ and therefore the composition $J=e \circ J^{\prime}$ is continuous. Notice also that the image of $J^{\prime}$ is contained in $C^{0}\left(\left(S^{1}, 0\right),(M, P)\right)=\Omega(M)$. It is clear that $J$ extends $j$.

We are now left to check that the framing associated to the embedding $i: Z \hookrightarrow S(P)$ also extends to a framing of $C \subset S(P) \times[0,1]$. We let $W(Q)=W^{s}(Q) \cap f^{-1}(-\infty, v]$. Of course $W(Q)$ is diffeomorphic to a disk embedded as manifold with boundary in the pair $\left(f^{-1}(-\infty, v], V\right)$. We fix the framing for the normal bundle of $W(Q)$. This means that we fix an ordered family $\left(s_{1}, \ldots, s_{q}\right)$ of linearly independent sections of this bundle such that they provide the fixed basis of $T_{Q} W(Q)$. The framing on the normal bundle of $Z \subset S(P)$ asociated to $i$ is obtained by projecting the sections $s_{i}$ on this normal bundle. For $t \geq 0$ let $W_{t}(Q)=h_{t}^{f}(W(Q))$. As $h_{t}^{f}$ is a diffeomorphism the normal bundle to $W_{t}(Q)$ is well defined and we can transport by $\left(h_{t}^{f}\right)_{*}$ the sections $s_{i}$ thus getting a framing of the normal bundle of $W_{t}(Q),\left(s_{1}^{t}, \ldots, s_{q}^{t}\right)$. We now consider the manifold $\bar{W} \subset M \times[0,1]$ defined by $\bar{W}=\cup_{0 \leq t \leq 1}\left(W_{t}(Q), t\right)$. We may define $q$ linearly independent sections in $T M \times\left.[0,1]\right|_{\bar{W}}$ by $\left(S_{i}\right)_{(x, t)}=\left(s_{i}^{t}\right)_{x} \times\{t\}$. We notice that this family of sections $\left(S_{1}, \ldots, S_{q}\right)$ generate a supplement of $T \bar{W}$ in $T(M \times[0,1])$. We now remark that $W^{\prime}$ is a submanifold of $\bar{W}$. Let $C^{\prime}=W^{\prime} \cap S^{\prime}$. Of course, $C^{\prime}=\phi^{\prime}(C)$. Project the sections $S_{i}$ onto the the normal bundle of $C^{\prime}$ in $\phi^{\prime}(S(P) \times[0,1])$. This produces $q$ linearly independent sections of this bundle $\left(S_{1}^{\prime} \ldots, S_{q}^{\prime}\right)$. We can transport these sections back to $C$ and they give a framing of $C$ inside $S(P) \times[0,1]$ which extends the framing of $Z \subset S(P)$ and cocludes the proof of the lemma.

We now proceed to the proof of Proposition 3.4

First we choose $\delta$ such that in the interval $(f(Q), f(Q)+\delta)$ there are no critical values of $\left.f\right|_{N}$ and $\delta \leq \delta^{\prime}$ for $\delta^{\prime}$ as in the proof of the last lemma. We 
fix $v$ inside this interval and, as before we let $V=f^{-1}(v)$. We now intend to adapt the proof of Lemma 3.6 to the present situation.

We preserve all notations from the proof of that lemma. The first remark is that $S(Q)$ has the same properties as in that proof. However, we need to replace $S(P)$ with a different space that takes into account the fact that there might exist critical points $R$ such that $f(Q)<f(R)<f(P)$. We let the space $\tilde{S}(P)$ consist of the points of $V$ that are situated on possibly broken, flow lines that originate in $P$. This space is compact but it is not in general a manifold. It will be necessary to understand some of its structure. For this let $R_{1}, \ldots, R_{h}$ be the critical points of $f$ such that $f\left(R_{i}\right) \in(f(Q), f(P))$ and $R_{i}$ is connected to $P$ by a possibly broken flow line of $\gamma(f)$. The MorseSmale condition implies that $\operatorname{ind}\left(R_{i}\right)<p$. Therefore, the maximal possible index of these critical points is $q$ (recall that by hypothesis $q$ and $p$ are succesive in $\left.\left\{k \in \mathbf{N}: \operatorname{Crit}_{k}^{N}(f) \neq \emptyset\right\}\right)$. We will assume that these critical points are ordered in decreasing order of their indexes so the first $s$ are all of index $q$ and the rest are of smaller indexes. If no $R_{i}$ has index $q$ we take $s=0$. The space $\tilde{S}(P)$ has the structure of a stratified space $\tilde{S}(P)=\cup F^{k_{i}}$ with the strata $F^{k_{i}}$ of dimension $k_{i}-1$ defined as follows: $F^{k_{i}}$ is the space of the points $x \in V$ that are situated on a non-broken flow line of $\gamma(f)$ originating in $P$ or in a critical point $R_{j}$ with $i n d\left(R_{j}\right)=k_{i}$. The first two top dimensional strata are therefore $F=F^{p}$ and $F^{q}=\cup_{1 \leq i \leq s}\left(W^{u}\left(R_{i}\right) \cap V\right)$. We denote $\bar{F}^{q}=\cup_{k_{i} \leq q} F^{k_{i}}$.

We now proceed as in the proof of Lemma 3.6. There is no modification in the construction of the regular index pair $\left(L_{1}, L_{0}\right)$ only that we now ask that $\tilde{S}(P)$ be contained inside $\operatorname{Int}\left(L_{1}-L_{0}\right)$. The definition of $W^{\prime}$ also remains the same. We let $\tilde{S}^{\prime}$ to be the image of an embedding $\tilde{\phi}^{\prime}$ close to the constant embedding $\tilde{S}(P) \times[0,1] \subset N_{1} \times[0,1]$ and such that $\tilde{\phi}^{\prime}$ is transvere to $W^{\prime}$ in the stratified sense (this means that each stratum is itself transverse to $\left.W^{\prime}\right)$. Of course, here $\tilde{S}(P) \times[0,1]$ is stratified with the strata $F^{k_{i}} \times[0,1]$. We denote $E^{k_{i}}=\tilde{\phi}^{\prime}\left(F^{k_{i}} \times[0,1]\right)$ and $\bar{E}^{q}=\tilde{\phi}^{\prime}\left(\bar{F}^{q}\right)$.

If the intersection $W^{\prime} \cap \bar{E}^{q}$ would be void, then the intersection $C^{\prime}=$ $W^{\prime} \cap \tilde{S}^{\prime}$ would equal $D=W^{\prime} \cap E^{p}$. Therefore, it would be a compact manifold providing a null-cobordism of $Z$ and the proof of Lemma 3.6 would apply whithout modification. However, $\operatorname{dim}\left(W^{\prime}\right)=n-q, \operatorname{dim}\left(\bar{E}^{q}\right)=q$ and transverality takes place inside $V \times[0,1]$ which is of dimension $n$. This means that in general $W^{\prime}$ might intersect $\bar{E}^{q}$ non-trivially. At the same time, transversality implies that $B=W^{\prime} \cap \bar{E}^{q}=W^{\prime} \cap E^{q}$ is a discrete union of points, $B=\left\{b_{1}, \ldots b_{r}\right\}$. Clearly, if we assume the condition (i) of the proposition, then $s=0, B=\emptyset$ and this argument shows that the desired conclusion holds.

To show that this is also true under the assumption (ii) we need to analyze the general situation. In this case $C^{\prime}$ is a stratified manifold with (regular) boundary and with two strata: the regular one, of dimension $p-q$, and the second one equal to $B$. Of course, $\partial C^{\prime}=Z$ and the same method as that 
used in the proof of Lemma 3.6 shows that we may extend the structure $(i, j)$ to $C^{\prime}-B$.

Our purpose now is to show that each point of $B$ has a neighbourhood in $C^{\prime}$ that is a cone over its boundary. We will then delete from $C^{\prime}$ the interior of a disjoint union of neighbourhoods of this type, one for each point of $B$. This will then provide a bordism between $Z$ and the disjoint union of the boundaries of these neighbourhoods. But we will also see that each such boundary is null-bordant.

We will now focus on a single critical point $R_{i}, 1 \leq i \leq s$, such that ind $\left(R_{i}\right)=q$. Let $S^{u}\left(R_{i}\right)=W^{u}\left(R_{i}\right) \cap V$ and $S^{s}\left(R_{i}\right)=W^{s}\left(R_{i}\right) \cap f^{-1}\left(f\left(R_{i}\right)+\right.$ $\left.\epsilon^{\prime}\right)$ such that $\epsilon^{\prime}$ is small enough such that there are no critical values in between $f\left(R_{i}\right)$ and $f\left(R_{i}\right)+\epsilon^{\prime}$. Of course, $S^{u}\left(R_{i}\right)$ is a submanifold in $V$ but it is not compact in general. Clearly, $S^{u}\left(R_{i}\right) \subset F^{q}$. It is not difficult to see that there is a neighbourhood $U\left(R_{i}\right)$ of $S^{u}\left(R_{i}\right)$ in $\tilde{S}(P)$ that is homeomorphic to $S^{u}\left(R_{i}\right) \times C\left(Z\left(P, R_{i}\right)\right)$ where $C(-)$ is the un-reduced cone. The key observation for this is that if one considers a tubular neighbourhood of $S^{s}\left(R_{i}\right)$ in $f^{-1}\left(f\left(R_{i}\right)+\epsilon^{\prime}\right)$ then the boundary of this neighbourhood intersects $W^{u}(P)$ along $S^{q-1} \times Z\left(P, R_{i}\right)$. The gradient flow of $f$ carries a sphere of the form $S^{q-1} \times p t \subset S^{q-1} \times Z\left(P, R_{i}\right)$ into a copy of $S^{u}\left(R_{i}\right)$ and therefore identifies the boundary of a neighbourhood of $S^{u}\left(R_{i}\right)$ in $\tilde{S}(P)$ to $S^{u}\left(R_{i}\right) \times Z\left(P, R_{i}\right)$. Let now $U^{\prime}\left(R_{i}\right)=\tilde{\phi}^{\prime}\left(U\left(R_{i}\right) \times[0,1]\right)$. Clearly, $U^{\prime}\left(R_{i}\right)$ is homeomorphic to $\left(S^{u}\left(R_{i}\right) \times[0,1]\right) \times C Z\left(P, R_{i}\right)$. We also have $E^{q}=\cup_{1 \leq i \leq s} \tilde{\phi}^{\prime}\left(S^{u}\left(R_{i}\right) \times[0,1]\right)$. Assume that the point $b_{j} \in W^{\prime} \cap E^{q}$ belongs to $\tilde{\phi}^{\prime}\left(S^{u}\left(R_{i}\right)\right)$. This means that $\left.b_{j} \in W^{\prime} \cap \tilde{\phi}^{\prime}\left(S^{u}\left(R_{i}\right) \times[0,1]\right)\right)$ and this last intersection is transverse. By trnasversality, the intersection $U^{\prime}\left(R_{i}\right) \cap W^{\prime}$ contains a neigbourhood $U_{b_{j}}$ of $b_{j}$ in $W^{\prime}$ which is homeomorphic to $C Z\left(P, R_{i}\right)$.

By eliminating from $C^{\prime}$ the disjoint union of the interiors of $U_{b_{j}}$ we obtain a manifold $C^{\prime \prime}$ with boundary such that $\partial C^{\prime \prime}$ is the disjoint union of $Z$ and a number of copies $Z_{i}^{j}=\partial U_{b_{j}}$ of some $Z\left(P, R_{i}\right), 1 \leq i \leq s$. As discussed above the structure $(i, j)$ extends to $C^{\prime \prime}$. The purpose now is to show that the restriction of this structure to $\amalg Z_{i}^{k}$ produces a bordism class which is the same with the sum of the bordism classes given by $j\left(P, R_{i}\right): Z\left(P, R_{i}\right) \rightarrow \Omega M$ together with the framings associated to $Z\left(P, R_{i}\right) \subset S^{u}(P)$. As all the stabilizations $\left[H\left(j_{P}, j_{R_{i}}\right)\right]$ of the Hopf invariants are vanishing by hypothesis, this implies that $Z$ is null-bordant and therefore, by Theorem 1 again, $\left[H\left(j_{P}, j_{Q}\right)\right]$ is null thus contradicting the hypothesis.

Let $J$ be the extension of the map $j$ to $C^{\prime \prime}$ constructed as in the proof of Lemma 3.6. It is easy to notice that $\left.J\right|_{Z_{i}^{k}} \simeq j\left(P, R_{i}\right)$ because, with the notations in the proof or 3.6 , we have that for all $z \in Z_{i}^{j}, w^{\prime \prime}(z)$ is arbitrarily close to a constant path joining $b_{j}$ to $Q$. On the other hand $w^{\prime}(z)$ is very close a path joining $P$ to $R_{i}$ and passing through $z^{\prime}$ where $z^{\prime}$ is identified to $z$ via the homeomorphism $Z_{i}^{j} \approx Z\left(P, R_{i}\right)$ and followed by a flow line from $R_{i}$ to $b_{j}$. 
The next task is to discuss the framings (this is the only place the nonvanishing of $\left[H\left(j_{P}, j_{Q}\right)\right]$ is needed and that of $H\left(j_{P}, j_{Q}\right)$ is not sufficient). For this consider $U\left(\bar{E}^{q}\right) \subset \tilde{S}^{\prime}$ to be a closed neighbourhood of the singular set $\bar{E}^{q}$ of $\tilde{S}^{\prime}$ of the from $\tilde{\phi}^{\prime}(U \times[0,1])$ with $U$ a neighbourhood of the singular set of $\tilde{S}(P)$ and such that $W^{\prime} \cap\left(\tilde{S}^{\prime}-U\left(\bar{E}^{q}\right)\right)=C^{\prime \prime}, \amalg Z_{i}^{j}=W^{\prime} \cap \partial U\left(\bar{E}^{q}\right)$ and $\amalg U_{b_{j}}=W^{\prime} \cap U\left(\bar{E}^{q}\right)$. Write the intersection point $b_{j} \in W^{\prime} \cap \tilde{S}^{\prime}$ as $b_{j}=$ $\left(a_{j}, t_{j}\right) \in V \times[0,1]$. By slighly modifying $W^{\prime}$ (using a compactly supported isotopy) in the neighbourhood of these intersection points we may assume that the $t_{j}$ 's are pairwise distinct and that $U_{b_{j}} \subset V \times\left\{t_{j}\right\}$. We assume that the $t_{j}>t_{j-1}$ for all the $b_{j}$ 's. Let $\delta>0$ be smaller than $t_{1}, 1-\delta>t_{r}$ and consider the manifold defined as $S^{\prime \prime}=\left(\tilde{S}^{\prime}-\operatorname{Int}\left(U\left(\bar{E}^{q}\right)\right)\right) \cup_{\partial U\left(\bar{E}^{q}\right)} D \times[0, \delta]$ where $D \subset S^{p-1}$ is such that $(\tilde{S}(P)-U) \cup D=S^{p-1}$. The existence of $D$ is verified by using the inverse flow of $f$ to move $\tilde{S}(P)-U$ inside $W^{u}(P) \cap f^{-1}\left(P-\epsilon^{\prime \prime}\right)$ for $\epsilon^{\prime \prime}$ very small. We can take $D$ to be the closure of the complement of the image of $\tilde{S}(P)-U$ inside this $p$-1-dimensional sphere. We now remark that, after rounding the corners of $S^{\prime \prime}$, we have that $S^{\prime \prime} \approx$ $S^{p-1} \times[0,1]$ via a homeomorphism such that $Z \subset \partial_{1} S^{\prime \prime} \approx S^{p-1} \times\{0\}$ and $\coprod Z_{i}^{j} \subset \partial_{2} S^{\prime \prime} \approx S^{p-1} \times\{1\}$. As in the proof of Lemma 3.6 we can construct $q$ linearly independent sections $\left(S_{1}^{\prime}, \ldots, S_{q}^{\prime}\right)$ of a supplement of $T W^{\prime}$ in $T(V \times$ $[0,1])$. These sections provide the framing of $Z$ in $\partial_{1} S^{\prime \prime}$. They provide a framing of $\coprod Z_{i}^{j}$ in $\partial_{2} S^{\prime \prime}$ and make $C^{\prime \prime}$ a framed cobordism inside $S^{\prime \prime}$. The key fact to recall now is that in general, unstably, the framing of a disjoint union is not equivalent to the sum of the framings of the components (two framings are equivalent if they are related by a framing of the product of the manifold with the unit interval). However, stably this is true - the (stable) bordism class of any disjoint union is just the sum of the bordism classes of the components. Therefore, we can now study the framing of each $Z_{i}^{j}$ at a time and, to conclude the proof of the proposition, it will be enough to show that the framing $\xi$ induced on $Z_{i}^{j}$ by the sections $\left(S_{k}^{\prime}\right)$ is equivalent at least stably with the standard framing of $Z\left(P, R_{i}\right) \subset S^{u}(P)$ (stable equivalence meaning that the two framings are equivalent after possibly adding a trivial bundle with a fixed framing to the two normal bundles in question). To simplify notation we may assume that in a neighbourhood $\mathcal{N}$ of $b_{j}$ in $V \times[0,1]$ we have $\tilde{\phi}^{\prime}\left(S^{u}\left(R_{i}\right) \times[0,1]\right) \cap \mathcal{N}=S^{u}\left(R_{i}\right) \times[0,1] \cap \mathcal{N}$. So for this argument we may assume $\tilde{\phi}^{\prime}\left(S^{u}\left(R_{i}\right) \times[0,1]\right)=S^{u}\left(R_{i}\right) \times[0,1]$. Recall that we have the inclusion $Z_{i}^{j} \hookrightarrow C Z_{i}^{j}=U_{b_{j}}$. Clearly, there is an obvious map $C Z_{i}^{j} \rightarrow b_{j}$ and, because the intersection of $W^{\prime}$ with $S^{u}\left(R_{i}\right) \times[0,1]$ is transverse in $b_{j}$ we have that the sections $\left(S_{k}^{\prime}\right)$ project to a basis in $T_{b_{j}}\left(S^{u}\left(R_{i}\right) \times[0,1]\right)$ which can be viewed as a section of the normal bundle of $b_{j}$ in $S^{u}\left(R_{i}\right) \times[0,1]$. By looking to the composition $Z_{i}^{j} \hookrightarrow C Z_{i}^{j} \rightarrow b_{j}$ we see that the framing of $Z_{i}^{j}$ is induced from this basis by this map (in the sense that we have maps between the total spaces of the normal bundles of the three spaces considered that make the sections correspond one to the other). Moreover, the contractibility of 
$C Z_{j}^{i}$ implies that any two framings induced in this way from two possibly different basis are equivalent.

We now need to use the relation between $Z_{i}^{j}$ and $Z\left(P, R_{i}\right)$. We fix $q$ sections $\left(x_{1}, \ldots, x_{q}\right)$ of the tangent bundle of $W^{u}\left(R_{i}\right)$. We can also extend them in the obvious way to a neighbourhood of $R_{i}$ contained in $W^{u}\left(R_{i}\right) \times$ $W^{s}\left(R_{i}\right)$. The value of these sections in $R_{i}$ induces the normal framing of $W^{s}\left(R_{i}\right)$ and that of $Z\left(P, R_{i}\right)$ in $S^{u}(P)$.

We recall that $\left\{a_{j}\right\} \times Z_{i}^{j} \subset S^{u}\left(R_{i}\right) \times\left(C Z_{i}^{j}-*\right) \subset\left(S^{u}\left(R_{i}\right) \times D^{n-q}\right) \cap$ $W^{u}(P)$ is obtained (by flowing via the negative gradient of $f$ ) from $\left\{a_{j}\right\} \times$ $Z\left(P, R_{i}\right) \subset S^{q-1} \times Z\left(P, R_{i}\right)=\partial D^{q} \times Z\left(P, R_{i} \subset W^{u}(P) \cap S^{s}\left(R_{i}\right)\right.$ (of course all this operation takes place in $M \times\left\{t_{j}\right\}$ but we will neglect this parameter). Fix the framing $\xi^{\prime}$ of the normal bundle of $\left\{a_{j}\right\} \times C Z_{i}^{j}$ inside

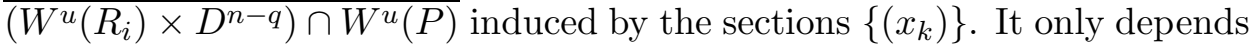
on the value of these sections in the point $\left\{a_{j}\right\} \times\{*\} \in S^{u}\left(R_{i}\right)$. At the same time the relation between $Z_{i}^{j}$ and $Z\left(P, R_{i}\right)$ means that $\xi^{\prime}$ is equivalent to the standard framing of $Z_{j}^{i}$ in $S^{u}\left(R_{i}\right)$.

We are now left to compare the framings $\xi$ and $\xi^{\prime}$ of the normal bundles of $C Z_{i}^{j}$ associated, the first to the stratified embedding $e_{1}:\left(C Z_{i}^{j}, *\right) \rightarrow$ $\left(\partial_{2} S^{\prime \prime}=S^{p-1} \times[0,1], S^{u}\left(R_{i}\right) \times[0,1]\right)$ and the second to the embedding

$\left.e_{2}:\left(C Z_{i}^{j}, *\right) \rightarrow \overline{\left(W^{u}(P)\right.}, W^{u}\left(R_{i}\right)\right)$. Both these two embeddings factor via the same embedding $\left(C Z_{i}^{j}, *\right) \rightarrow\left(\tilde{S}(P), S^{u}\left(R_{i}\right)\right)$ and the framings are induced in the way described before by pull-back over the composition $Z_{i}^{j} \hookrightarrow\left(C Z_{i}^{j}, *\right) \rightarrow *$. After adding a rank one trivial line bundle to the two normal bundles in question we see that $\xi$ and $\xi^{\prime}$ become equivalent. This concludes the proof of the proposition.

\section{Proof of Theorem 0 .}

We assume that $f, P$ and $Q$ are as in the statement. As $\left[H\left(j_{P}, j_{Q}\right)\right] \neq$ 0 there is some critical point $Q^{\prime} \in \operatorname{Crit}_{q}^{N}(f)$ such that $\left[H\left(j_{P}, j_{Q^{\prime}}\right)\right] \neq 0$, $f\left(Q^{\prime}\right) \geq f(Q)$ and for all $R \in \operatorname{Crit}_{q}^{N}(f), f\left(Q^{\prime}\right)<f(R)<f(P)$ we have $\left[H\left(j_{P}, j_{R}\right)\right]=0$. We can therefore apply Proposition 3.4 to the pair $P, Q^{\prime}$. We obtain that there exists some $\delta>0$ such that there are infinitely many regular values $v_{i} \in(f(Q)+\delta, f(Q)+2 \delta)$ (of $\left.\left.f\right|_{N}\right)$ such that each hypersurface $V_{v_{i}}=f^{-1}\left(v_{i}\right) \cap N_{1}$ contains at least one bounded orbit of $h^{f}$. We may assume that the set $\left\{v_{i}\right\}$ is discrete. We fix one such $v_{i}$. Consider a point $x \in V_{v_{i}}$ whose orbit is bounded. Then $\omega(x)$ is non-void. Let $y \in \omega(x)$. Then $y$ is non-wandering and belongs to $\Omega_{c}\left(h^{f}\right)$. We now fix some small $\epsilon_{i}>0$ and apply the $C^{1}$ closing lemma of Pugh and Robinson [21] to deform $H_{f}$ by a small $C^{1}$ deformation with a fixed compact support (only depending on the orbit to be closed) contained in $f^{-1}\left(v_{i}-\epsilon_{i}, v_{i}+\epsilon_{i}\right)$ to obtain a hamiltonian vector field $H_{f^{\prime}}$ such that $h^{f^{\prime}}$ has a periodic orbit close to $y$. Because the deformation used has a fixed compact support, $f^{\prime}$ can be made as $C^{2}$-close 
to $f$ as needed and such that the constructed periodic orbit is included in $f^{-1}\left(v_{i}-\epsilon_{i}, v_{i}+\epsilon_{i}\right)$. We can then apply the same process for all the values $v_{i}$ by taking the $\epsilon_{i}$ 's such that the intervals $f^{-1}\left(v_{j}-\epsilon_{j}, v_{j}+\epsilon_{j}\right)$ are pairwise disjoint, $\lim _{j \rightarrow \infty} \epsilon_{j}=0$ and, when $j \rightarrow \infty$, the deformations used tend to 0 . This leads to the existence of the function $\bar{f}$ and concludes the proof.

\subsection{Applications and examples.}

The first paragraph below is concerned with a disscussion of property $(*)$. The main part of the sub-section appears in the second paragraph which contains corollaries of Theorem 2. We end with some open problems and further questions.

\subsubsection{Property $(*)$.}

A few simple critera that insure the validity of property $(*)$ are contained in the following lemma.

Lemma 3.7. Let $f: M \rightarrow \mathbf{R}$ be a $C^{2}$ function. Let $S$ be an isolated invariant set for the negative gradient flow $\gamma=\gamma(f)$ of $f$. The invariant set $S$ satisfies property $(*)$ if one of the following conditions is satisfied.

(i) There exist an isolating neighbourhood $N$ of $S, v \in \mathbf{R}$ regular for $\left.f\right|_{\text {Int }(N)}$ and a neighbourhood $U \subset N$ of $W_{N}^{s}(S)$ such that $v<\inf \{f(x)$ : $x \in S\}$ and, for all $x \in U-W_{N}^{s}(S)$, there is $t \in \mathbf{R}^{+}$with $f\left(\gamma_{t}(x)\right)=v$, $\gamma_{[0, t]}(x) \subset N$.

(ii) There are regular values $a<b$ of $f$ such that $S$ is the maximal compact invariant set contained in $f^{-1}([a, b])$ and for any $x \in f^{-1}([a, b])$ we have: $\omega(x) \subset S$ or there exists some $t \in \mathbf{R}^{+}$such that $f\left(\gamma_{t}(x)\right)=a$ and $\omega^{*}(x) \subset S$ or there is $t \in \mathbf{R}^{+}, f\left(\gamma_{-t}(x)\right)=b$.

(iii) $S$ is the maximal compact invariant set of $\gamma$, there exists a compact $K \in M$ and an $\epsilon>0$ with $\|\nabla f(x)\|>\epsilon$ for $x \notin K$ and $\gamma$ is a flow (not only a partially defined one).

(iv) $S$ is the maximal compact invariant set of $\gamma, M$ is metrically complete and there exist $m>\epsilon>0$ such that outside some compact set we have $m>\|\nabla f(x)\|>\epsilon$.

Proof. It is clear how to use (i) to prove property (*): we consider $U^{\prime} \subset$ $\operatorname{Int}(U)$ another neighbourhood of $W_{N}^{s}(S)$ and we take $N_{1}=\{x \in N: \exists t>$ $\left.0, \gamma_{[-t, 0]}(x) \subset N, \gamma_{-t}(x) \in \overline{U^{\prime}}\right\}$ and we let $N_{0}=N_{1} \cap f^{-1}(v)$. From (ii) we immediately deduce that we may construct $N, U$ as required at (i). Point (iii) implies that the condition at (ii) is verified; (iv) implies (iii).

Remark 3.8. It is immediate that condition (i) in the lemma above is, in fact equivalent to property $(*)$.

It is easy to produce examples when condition $(*)$ is not satisfied.

Example 2. Let $g: D^{2} \rightarrow \mathbf{R}$ be a Morse-Smale function with precisely two critical points: 0 which is of index 2 and $Q \in \operatorname{Int}\left(D^{2}\right)$ of index 1 . We may also find $g$ such that $g(0)=1, g(Q)=0$ and there is precisely one (negative 
gradient) flow line $\xi$ in $D^{2}$ that joins 0 to $Q$. We consider $D^{\prime}=D^{2}-\{T\}$ where $T$ is a point in $D^{2}$ which is not in $\xi \cup\{0, Q\}$ and $g(T)>0$. We let $f=\left.g\right|_{D^{\prime}}$ and take $S=\{0, Q\} \cup \xi$. It is easy to check that $S$ does not have the property $(*)$.

A simple result concerning this property $(*)$ will be of use later. We recall that $\mathcal{F}_{l}(M)$ is the space of $C^{2}$ local isolated functions on $M$, that is pairs $(f, N)$ with $f$ a $C^{2}$ function and $N$ an isolating neighbourhood of the negative gradient flow $\gamma(f)$ of $f$.

Lemma 3.9. Let $(f, N) \in \mathcal{F}_{l}(M)$ and $S=I_{N}(f)$. Suppose that $S$ verifies property $(*)$. There is a $C^{2}$-neighbourhood $\mathcal{U}$ of $f$ such that if $g \in \mathcal{U}$, then $(g, N) \in \mathcal{F}_{l}(M)$ and $I_{N}(g)$ verifies $(*)$.

Proof. Fix $\left(N_{1}, N_{0}, N_{2}\right)$ a strong index block of $S$ with $N_{0} \subset f^{-1}(a)$ and $f$ regular on $N_{0}$. It is clear that by taking $\mathcal{U}^{\prime}$ a sufficiently small neighbourhood of $g$ we have that $\left(N_{1}, N_{0}, N_{2}\right)$ is also a strong index block for each $g \in \mathcal{U}^{\prime}$. Fix a regular value of $\left.f\right|_{N_{1}}, a^{\prime}>a$ such that $a^{\prime}<\inf \{f(S)\}$. It is easy to see that by possibly diminishing more $\mathcal{U}^{\prime}$ the pair $\left(N_{1} \cap g^{-1}\left(\left[a^{\prime}, \infty\right)\right), N_{1} \cap g^{-1}\left(a^{\prime}\right)\right)$ becomes an index pair for each such $g$.

\subsubsection{Corollaries of Theorem 园.}

We start by defining the invariant $d(-)$ mentioned in the introduction. This invariant is inspired by the fact that in the statement of Theorem 2 the Hopf invariant appearing there is a coefficient in the differential of the extended Morse complex $\mathcal{C}_{N}(f)$.

Definition 7. Let $(f, N) \in \mathcal{F}_{l}(M)$. If $(f, N)$ is a local Morse function we let $d_{N}(f) \in \mathbf{Z} / 2$ be equal to 1 iff $\mathcal{C}_{N}(f)$ has a non-trivial differential. For a general $(f, N) \in \mathcal{F}_{l}(M)$ we let $d_{N}(f)=\sup \left\{\inf \left\{d_{N}(g): g \in\right.\right.$ $\mathcal{U},(g, N)$ local Morse $\}: \mathcal{U}$ open in $\left.C^{2}(M, \mathbf{R}), f \in \mathcal{U}\right\}$ (here $C^{2}(M, \mathbf{R})$ is the space of $C^{2}$ functions with the strong topology).

Clearly, if $d_{N}(f)=1$, then all Morse functions $g$ which are sufficientely close to $f$ have an extended Morse complex $\mathcal{C}_{N}(g)$ with non-trivial differential. Moreover, there is a neighbourhood of $f$ consisting of functions $f^{\prime}$ that have $N$ as an isolating neighbourhood and with $d_{N}\left(f^{\prime}\right)=1$.

The local isolated functions $(f, D)$ with $f$ appearing in Example 1 and $D \subset \mathbf{R}^{2 k}$ a closed disk containing the origin verify $d_{D}(f)=0$.

Assume now that $f$ is a $C^{2}$ function and $S$ is an isolated invariant set for $\gamma(f)$. We let $d(S)=d_{N}(f)$ where $N$ is an isolating neighbourhood such that $S=I_{N}(f)$. It is immediate that $d(S)$ is indeed independent of $N$. We also let $e(S)$ to be the infimum of $d_{N^{\prime}}\left(f^{\prime}\right)$ where the pair $\left(f^{\prime}, N^{\prime}\right) \in \mathcal{F}_{l}(M)$ is continuation equivalent to $(f, N)$ for some $N$ with $S=I_{N}(f)$. Assuming $(f, N) \in \mathcal{F}_{l}(M)$ fixed, notice, as above, that a function $f^{\prime}$ that is sufficiently close to $f$ has $N$ as an isolating neighbourhood and $\left(f^{\prime}, N\right)$ is continuation equivalent to $(f, N)$. This means that $d(S) \geq e(S)$. It is very easy to construct examples such that $d(S)>e(S)$. For example, take $S=\{0, Q\} \cup \xi$ in Example 2. 
Here is an important consequence of Theorem 2.

Corollary 3.10. Let $(f, N) \in \mathcal{F}_{l}(M)$ such that $S=I_{N}(f)$ satisfies property $(*)$ and $d(S)=1$.

(i) If $(f, N)$ is a local Morse function, then there are infinitely many regular hyersurfaces $V=f^{-1}(v)$ which, after perturbation by an arbitrarily small compactly supported isotopy of $M$ carry a closed characteristic.

(ii) There is a family of functions, $\mathcal{F}$, dense in a $C^{2}$ neighbourhood of $f$ such that each hamiltonian flow $h^{f^{\prime}}, f^{\prime} \in \mathcal{F}$ has infinitely many distinct periodic orbits.

Proof. We prove point (i). We first show the existence of one hypersurface $V$. If $(f, N)$ is a local Morse function and $\mathcal{C}_{N}(f)$ has a non-trivial diferential we obtain that there is a pair of consecutive critical points $P, Q \in \operatorname{Crit}^{N}(f)$ verifying the assumption of Theorem 2. By the proof of this theorem we obtain that we may find a regular value $v$ of $f$ such that in any $C^{2}$ neighbourhood of $f$ and for any $\epsilon$ there is a function $\bar{f}$ whose associated hamiltonian has inside $\operatorname{Int}(N) \cap \bar{f}^{-1}(v-\epsilon, v+\epsilon)$ at least one periodic orbit. Assume that this periodic orbit actually belongs to $\bar{f}^{-1}\left(v^{\prime}\right)$. By taking $\epsilon$ small enough and $\bar{f}$ very close to $f$ it is easy to see that there is a compactly supported isotopy $\phi$ of $M$ such that $\phi(V) \cap \operatorname{Int}(N)=\bar{f}^{-1}\left(v^{\prime}\right) \cup \operatorname{Int}(N)$. This means that the hypersurface $V^{\prime}=\phi(V)$ carries a closed characteristic. Now the same argument can be applied for infinitely many hypersurfaces $V$ as in the proof of Theorem 2.

The second point is essentially obvious given Theorem 2. By Lemma 3.9 all the functions in a sufficiently small neighbourhood $\mathcal{U}$ of $f$ are such that $g \in \mathcal{U}$ implies $(g, N) \in \mathcal{F}_{l}(M)$ and $I_{N}(g)$ verifies property $(*)$. As seen above me may assume $\mathcal{U}$ small enough such that each $g \in \mathcal{U}$ also satisfies $d\left(I_{N}(g)\right)=1$. Local Morse functions form a dense family in $\mathcal{U}$. For such a function $(h, N)$ we have $d_{N}(h)=1$ which means that $\mathcal{C}_{N}(h)$ has a non trivial differential. But by Theorem 1 this means that the assumptions needed to apply Theorem 2 are satisfied. Therefore arbitrarily close to $h$ there is a function $h^{\prime}$ whose hamiltonian flow has infinitely many periodic orbits. This proves the claim at (ii).

Remark 3.11. It is useful to note that the hypersurfaces produced at the point (i) of Corollary 3.10 might all be isotopic one to the other.

It is hard in general to determine $d(f)$. Of course, the exact determination of $e(f)$ is not simple either. However, and this is fundamental for applications, we have a reasonably effective criterion that insures $e(f)=1$.

Corollary 3.12. Let $(f, N) \in \mathcal{F}_{l}(M)$ and $S=I_{N}(f)$. Each one of the following conditions implies that $e(S)=1$. 
(i) There exists $\left(g, N^{\prime}\right)$ which is local Morse, continuation equivalent to $(f, N)$ and such that the differential $d_{g}$ in $\mathcal{C}(g)$ verifies $d_{g} \neq d^{M o} \otimes$ $i d_{\Omega M}$.

(ii) There is no minimal local Morse function that is continuation equivalent to $(f, N)$

(iii) There is a minimal Morse function $\left(g, N^{\prime}\right)$ continuation equivalent to $(f, N)$ and its extended Morse complex $\mathcal{C}_{N^{\prime}}(g)$ has a non-trivial differential.

(iv) $H_{*}\left(c_{N}(f) ; \mathbf{Z}\right)$ has torsion.

(v) $H_{*}\left(c_{N}(f) ; \mathbf{Z}\right)$ is torsion free, $\bar{c}_{N}(f)$ is simply-connected and there are $k, l \in \mathbf{N}$ with $H_{t}\left(c_{N}(f) ; \mathbf{Z}\right)=0$ for $0<k<t<l, l-k>1$ and in the homology Serre (or in the $\left.\pi_{*}^{S}(\Omega M)^{+}\right)$- Atiyah-Hirzebruch-Serre) spectral sequence of the fibration induced by the canonical projection (see $\S 1.1) \bar{c}_{N}(f) \stackrel{p}{\rightarrow} M$ from $\Omega M \rightarrow P M \rightarrow M$ the differential $d^{l-k}$ (resp. $D^{l-k}$ ) does not vanish when restricted to $E_{l *}^{l-k}$ (resp. $\mathcal{E}_{l *}^{l-k}$ ).

Proof. For any local Morse function $\left(g, N^{\prime}\right)$ the differential $d^{g}$ of the complex $\mathcal{C}_{N^{\prime}}(g)$ contains $d^{M o}$ in the sense that $\left(d^{M o} \otimes i d_{\Omega M}\right)(x)=d^{g}(x)$ for all critical points $x$ such that $C r i t_{|x|-1}^{N^{\prime}}(g) \neq \emptyset$. Therefore, $d^{M o} \neq 0$ implies $d^{g} \neq 0$. This means that if $g$ is not minimal, then $d(g)=1$. Therefore, to decide that $e(S)=1$ we only need to make sure that each minimal local Morse function $\left(g, N^{\prime}\right)$ which is continuation equivalent to $(f, N)$ verifies $d_{N^{\prime}}(g)=1$. But by Corollary 2.18 any two minimal Morse functions that are continuation equivalent have isomorphic extended Morse complexes. Thus it is enough to verify this condition for just one minimal local Morse function that is continuation equivalent to $(f, N)$.

This remark directly justifies our claim if one of the conditions (ii),(iii) or (iv) is satisfied. It also implies the claim if (v) is satisfied. Indeed, asuming (v) and supposing that a minimal local Morse function $\left(g, N^{\prime}\right)$ is continuation equivalent to $(f, N)$ its Morse complex $\mathcal{C}_{N^{\prime}}(g)$ is isomorphic by Corrolary 2.19 to the chain complex $\mathcal{C}_{\min }\left(\bar{c}_{N}(f)\right.$ ) (which is defined under the assumptions made). At the same time Corollary 2.7 shows that the differential of this last complex is non-trivial.

We are now left to prove that the point (i) also implies $e(S)=1$. The condition at (i) implies that there is some critical point $P \in \operatorname{Crit}^{N^{\prime}}(g)$ whose differential in the classical Morse complex is null but its differential in $\mathcal{C}_{N^{\prime}}(g)$ is not vanishing. But this means that $P$ represents a non-trivial homology class in the homology of the classical Morse complex and that there is another critical point $Q \in \operatorname{Crit}^{N^{\prime}}(g)$ such that $[H(P, Q)] \neq 0$. It also results that if $q=i n d_{g}(Q)$ and $p=i n d_{g}(P)$, then $p>q+1$. As explained before we need to consider the case when there exists a minimal local Morse function $\left(h, N^{\prime \prime}\right)$ continuation equivalent to $(f, N)$ (and thus to $\left.\left(g, N^{\prime}\right)\right)$ and it is enough to show that $d_{N^{\prime \prime}}(h)=1$. We obtain by Corollary 2.16 that there exists a map $m: C_{N^{\prime}}^{M o}(g) \rightarrow C_{N^{\prime \prime}}^{M o}(h)$ which induces a morphism of extended Morse complexes for all compatible index sets. Let 
$I=\{0,1, \ldots, q-1, q, p, p+1, \ldots, n\}$. Then both complexes $\mathcal{C}_{N^{\prime}}^{I}(g)$ and $\mathcal{C}_{N^{\prime \prime}}^{I}(h)$ are defined. Denote by $m^{I}$ the morphism induced by $m$ between these two extended complexes. We denote by $\tilde{d}$ the differential in these two complexes. As $m$ induces an isomorphism in homology and $P$ represents a non-trivial homology class in $H_{*}\left(C^{M o}(g)\right)$ it results that there is $u \in \mathbf{Z}\left[\operatorname{Crit}_{p}^{N^{\prime \prime}}(h)\right]$ such that $m(u)=P+d^{M o}(b)$ for some $b \in C_{N^{\prime}}^{M o}(g)$ and $d^{M o}(u)=0$ because $p>q+1$ and $h$ is minimal. Now, we have that $\tilde{d} d^{M o}=0$. Therefore $\tilde{d}\left(m^{I}(u)\right)=\left[H\left(j_{p}, j_{Q}\right] Q+c\right.$ where $c$ does not contain any terms in $Q$. Therefore, $\tilde{d}\left(m^{I}(u)\right) \neq 0$. But this implies that $\tilde{d} u \neq 0$. Therefore the differential $\tilde{d}$ is not zero in $\mathcal{C}_{N^{\prime \prime}}^{I}(h)$. But the differential in $\mathcal{C}_{N^{\prime \prime}}(h)$ contains $\tilde{d}$ and therefore is also non-trivial.

Remark 3.13. a. The conditions (iv), (v) appearing in Corollary 3.12 are continuation invariant. However, sometimes it is easier to apply a version of these which uses instead of the pair $\bar{c}_{N}(f)$ an index pair $\left(N_{1}, N_{0}\right)$ of the invariant set $I_{N}(f)$. In this case condition (iv) remains the same for recall that $H_{*}\left(c_{N}(f) ; \mathbf{Z}\right)=H_{*}\left(N_{1}, N_{0} ; \mathbf{Z}\right)$. At point $(\mathrm{v})$ the assumption that replaces the simple-connectivity of $\bar{c}_{N}(f)$ is that both $N_{1}$ and $N_{0}$ are simply connected. Of course, instead of the spectral sequences associated to the pair $\bar{c}_{N}(f)$ one may use the same spectral sequences for the pair $\left(N_{1}, N_{0}\right)$ Finally, in view of Corollary 2.7, this spectral sequence condition as it appears at point (v) is equivalent with the fact that the minimal Hopf complex of the pair $\left(N_{1}, N_{0}\right)$ has a non trivial differential.

b. It is easy to find manifolds $M$ and Morse functions $f: M \rightarrow \mathbf{R}$ such that condition (i) of the Corollary 3.12 is satisfied (of course, $\pi_{*}^{S}\left(\Omega M^{+}\right.$) needs to be nontrivial for $*>0$ ). A simple example is provided by the height function $h$ on a sphere $S^{k}$ for $k \geq 1$. This has precisely two critical points: $T$ a maximum and $S$ a minimium. The differential in the classical Morse complex is null. However, in $\mathcal{C}_{S^{k}}(h)$ we have $d T=(I d)^{*}$ where $(I d)^{*}$ is the stable homotopy class of the inclusion of the bottom cell $S^{k-1} \hookrightarrow \Omega S^{k}$. Another instructive example is a minimal Morse function $\tilde{h}$ on $\mathbf{C} P^{n}$. Again, the classical Morse differential vanishes but on each critical point $P$ of $\tilde{h}$ with $\operatorname{ind}(P)>0$ the differential in the extended Morse complex $\mathcal{C}(\tilde{h})$ is not null. Of course, both these examples are compact but via a product with some non-compact manifold ( $\mathbf{R}^{k}$, for example) or, more generally, by considering some vector bundles over them, they produce non-compact variants.

The Serre spectral sequence condition at (v) is in many cases easy to verify.

Example 3. Let $M=\mathbf{C} P^{n} \times \mathbf{R}^{2 k}$ and consider the function $g \oplus q$ where $g: \mathbf{C} P^{n} \rightarrow \mathbf{R}$ is a perfect Morse function and $q: \mathbf{R}^{2 k} \rightarrow \mathbf{R}$ is a quadratic form of index different from 0 or $2 k$. Fix on $M$ an arbitrary symplectic form and let $f$ be obtained by a compactly supported perturbation of $g \oplus q$ (notice that if we take on $M$ a symplectic form that is simply the sum of 
the forms on $\mathbf{C} P^{n}$ and $\mathbf{R}^{2 k}$, then it is obvious that $h^{g \oplus q}$ has many bounded orbits because the hamiltonian flow leaves invariant $\left.\mathbf{C} P^{n} \times\{0\} \subset M\right)$.

The gradient flow of $f$ has a maximal compact invariant set $I(f)$ and it is easy to see that this set satisfies the condition $(*)$ (this follows by applying (ii) of Lemma 3.7). Notice also that $d(I(f))=1$. Indeed, there exists an index pair of $I(f)$ of the form $\left(N_{1}, N_{0}\right)=\left(\mathbf{C} P^{n} \times D^{2 k}, \mathbf{C} P^{n} \times S^{j-1} \times D^{2 k-j}\right)$ where $j=\operatorname{ind}(q)$. Consider the homology Serre spectral sequence $\left(E_{p q}^{r}, d^{r}\right)$ of the fibration $\Omega \mathbf{C} P^{n} \rightarrow\left(E_{1}, E_{0}\right) \rightarrow\left(N_{1}, N_{0}\right)$ which is obtained by pullback of the (homotopy) fibration $\Omega \mathbf{C} P^{n} \rightarrow * \rightarrow \mathbf{C} P^{n}$. It is immediate to see that this spectral sequence is simply a suspension of the Serre spectral sequence $\left(e_{p q}^{r}, \delta^{r}\right)$ of this last fibration in the sense that $E_{(p+j) q}^{r}=e_{p q}^{r}$ and similarly for the differentials. In turn this fibration has all differentials $\delta_{(2 l) 0}^{2}$ non trivial. This means that the conditions needed to apply Corollary 3.12 (v) are satisfied.

This example also indicates that the non-vanishing of $d(-)$ is sometimes implied by a non-trivial cup-structure in co-homology (this is of course to be expected as the non-vanishing of Hopf invariants is implied by the existence of certain non-trivial cup-products).

Some of the conditions in the last corollary imply slightly more than what is claimed there. The next result also emphasizes the stability properties of the extended Morse complexes.

Corollary 3.14. Let $(f, N) \in \mathcal{F}_{l}(M)$ and let $\left(g, N^{\prime}\right) \in \mathcal{F}_{l}\left(M \times \mathbf{R}^{2 k}\right)$ such that $\left(g, N^{\prime}\right)$ is continuation equivalent to $\left(f \oplus q, N \times D^{2 r}\right)$ with $q: \mathbf{R}^{2 r} \rightarrow \mathbf{R}$ a non-degenerate quadratic form. If one of the conditions (iv) and ( $v$ ) from Corollary 3.19 are satisfied for $(f, N), S=I_{N}(f)$, then there exists a family of functions dense in a neighbourhood of $g$ each of whose induced hamiltonian flow on $M \times \mathbf{R}^{2 r}$ (endowed with an arbitrary symplectic form) has infinitely many distinct periodic orbits.

Proof. Condition (iv) shows that there are no minimal Morse functions in the continuation equivalence class of the sum $\left(f \oplus q, N \times D^{2 r}\right)$ (because the Conley index of the maximal invariant set associated to such a function is just a suspension of the Conley index asssociated to $(f, N))$. Similarly, by the definition of the global Conley index, we see that $\bar{c}_{N \times D^{2 r}}(f \oplus q)$ is simply connected and torsion free and therefore the minimal Hopf complex $\mathcal{C}_{\text {min }}\left(\bar{c}_{N \times D^{2 r}}(f \oplus q)\right)$ is defined. It is easy to see that this complex is just the suspension of order $\operatorname{ind}(q)$ of $\mathcal{C}_{\min }\left(\bar{c}_{N}(f)\right)$. Thus, as condition (v) implies that the differential in this last complex is not trivial, we obtain that the differential of the first is not trivial either. This immediately implies the claim by using Corollary 3.12 .

We now present a class of examples where the methods decribed above apply naturally.

Example 4. Let $B$ be a simply connected, closed manifold, such that $B \times \mathbf{R}^{n}$ is symplectic. Suppose that $f: B \times \mathbf{R}^{n} \rightarrow \mathbf{R}$ is a smooth function that is 
quadratic at infinity. Then $f$ satisfies the conclusion of Corollary 3.10 (ii). If $f$ is a Morse function, then it satisfies the conclusion of Corollary 3.10 (i).

We recall that a function $f$ is quadratic at infinity if there is a compact set $K \subset B \times \mathbf{R}^{n}$, a smooth function $g: B \rightarrow \mathbf{R}$ and a non-degenerate quadratic form $q: \mathbf{R}^{n} \rightarrow \mathbf{R}$ of index $k, 0 \leq k \leq n$ such that $f(x, y)=g(x)+q(y)$ for $(x, y) \notin K$.

The proof of the claim follows from the fact that for a sufficientely large disk $D \subset \mathbf{R}^{n}$ the set $B \times D$ is an isolating neighbourhood for $\gamma(g \oplus q)$ and it is easy to see that for a big $D,(f, B \times D)$ is continuation equivalent to $(g+q, B \times D)$. Moreover, the flow $\gamma(f)$ is defined for infinite time and this implies that $S=I_{B \times D}(f)$ verifies property (*) by Lemma 3.7 (iii). We also see $d(S)=1$ which is obtained by applying Corollary 3.12. Indeed, if there is a minimal Morse function continuation equivalent to $\left(g+q, B \times D^{n}\right)$ one look to the relevant Serre spectral sequence suffices to apply 3.12 (v).

Of course, the result is trivial if $k=0$ or $k=n$ because in these two cases the compactness of $B$ implies that the level surfaces of $f$ are also compact.

Many examples of symplectic manifolds $B \times \mathbf{R}^{n}$ are such that $B$ is itself symplectic and the symplectic form on the product is the product one. However, there are many examples that are not of this type (one such is the cotangent bundle of the three sphere) and in all cases the result mentioned is true for an arbitrary symplectic form on the product.

There are many ways in which to extend this result. We list below a few such possiblities (that can be also mixed).

(i) Take $B$ non compact but $g$ such that there is some invariant set for the negative gradient flow of $g$ that satisfies the conditions (iii) or (iv) in Corollary 3.12

(ii) Replace $q$ with a function $h: M^{\prime} \rightarrow \mathbf{R}$ such that the gradient of $h$ induces a flow on $N$, every level hypersurface of $h$ is not compact, the critical set of $h$ is not trivial and outside of some compact in $N$ the gradient of $h$ is bounded away from 0 .

(iii) Replace $B \times \mathbf{R}^{n}$ with the total space of a fibre bundle of basis $B$ that is not trivial.

Given the result above it is easy to produce non-compact hypersurfaces $V$ with no compact connected components such that after a small isotopy they carry a closed characteristic. Let $M=S^{3} \times \mathbf{R}^{3}=\{(x, y, z, w, m, n, p)$ : $\left.x^{2}+y^{2}+z^{2}+w^{2}=1\right\}$. Take $V \subset M$ be defined by $V=\{(x, y, z, w, m, n, p)$ : $\left.x+m^{2}+n^{2}-p^{2}=1 / 2\right\}$. The symplectic structure on $M$ comes from the identification $M \approx T^{*} S^{3}$. By a slight variation we may also construct examples of manifolds $M$ that contain connected non-compact hypersurfaces $V_{1} \ldots V_{k}$ pairwise non homeomorphic and such that after a small isotopy each contains a closed characteristic. Whenever Corollary $3.12(\mathrm{v})$ applies, the number of such hypersurfaces is (essentially) estimated by half the number of pairs $k, l \in \mathbf{N}$ which verify the condition (v) in the corollary. For example, if $M=S^{3} \times S^{3} \times S^{3} \times \mathbf{R}^{9}$ (which is symplectic as $M \approx T^{*} S^{3} \times T^{*} S^{3} \times$ $\left.T^{*} S^{3}\right)$ we can find in $M$ at least two hypersurfaces that are non-compact, 
connected and non-homeomorphic that carry a closed characteristic after a small deformation.

\subsubsection{Final comments and open questions.}

A. The one major question that is left open by this paper is to find some analytic assumptions which, once imposed to an $(f, N) \in \mathcal{F}_{l}(M)$ which already satisfies one of the homotopical conditions in Corollary 3.12, will imply that $h^{f}$ has some periodic orbits. One could hope to unify the results on the existence of periodic orbits which are valid in the compact setting with those that work in the non-compact case. We should point out however that the major defficiency of the existence results that we described in the non-compact situation is not so much their validity only generically but rather the fact that, on one hand, we do not have any control on the periods of the orbits constructed, and, on the other hand, the genericity assumption involved is not "verifiable" (by contrast to the form of genericity proper to Floer theory where it is determined by the behaviour of a certain Fredholm operator).

B. Of less significance but still of interest are the following questions:

(i) For $(f, N) \in \mathcal{F}_{l}(M)$ fixed assume that there is a minimal Morse function $\left(g, N^{\prime}\right)$ continuation equivalent to $(f, N)$ but $\mathcal{C}_{N^{\prime}}(g)$ has a vanishing differential (in other words all the relevant relative Hopf invariants vanish). Find some other homotopical condition on $f$ that depends on some higher homotopy operation (like Massey products or Toda brackets) in $\pi_{*}^{S}\left(\Omega M^{+}\right)$ that is sufficient for the existence of a function arbitrarily close to $g$ whose hamiltonian flow has infinitely many periodic orbits. The first stage would be to extend Theorem 1 for these higher homotopy operations by relating them to bordism classes of "maniflods" of possibly broken connecting flow lines. The second stage would be to extend the argument in Theorem 2 to the case of these bordism classes.

(ii) Extend the arguments in theorems 1, 2 for various other classes of functions, not necessarily Morse. For example functions having only "neat" singularities as in [6] or Morse-Bott functions.

C. Here is a possibly useful extension of Theorem 1 that is not hard to obtain but has not been explicitely included to avoid a considerable increase in technical difficulty. The statement is similar to that of the theorem but applied to the Thom map of $\coprod_{i} Z\left(P, Q_{i}\right) \hookrightarrow S^{u}(P)$ where $\operatorname{ind}(P)=p>q=$ $\operatorname{ind}\left(Q_{i}\right)$ and $\operatorname{Crit}_{k}^{N}(f)=\emptyset$ for $q<k<p$. This Thom map is equal to the relative Hopf invariant, $H\left(P,\left\{Q_{i}\right\}\right)$, obtained from two succesive cofibrations $\vee_{i} S_{i}^{q-1} \rightarrow N^{\prime} \rightarrow N^{\prime \prime}$ and the second $S^{p-1} \rightarrow N^{\prime \prime} \rightarrow N^{\prime \prime \prime} \subset M$ the first corresponding to the simultaneous cell-attachements corresponding to the $Q_{i}$ 's and the second to the cell attachement corresponding to $P$. Unstably, this Thom map is richer than the sum of the Thom maps corresponding 
to the pairs $\left(P, Q_{i}\right)$ but stably it equals this sum. In the statement of Proposition 3.4 (i) one can use $H\left(P,\left\{Q_{i}\right\}\right)$ instead of $H\left(j_{P}, j_{Q}\right)$.

\section{REFERENCES}

1. H.Baues, Homotopy Type and Homology, Oxford Press 1996

2. Ch.Conley, Isolated invariant sets and the Morse index, CBMS Regional Conf.Ser. in Math. 38 Amer. Math. Soc., Providence, R. I. (1976)

3. O.Cornea, Cone-length and Lusternik-Schnirelmann category, Topology 33 (1994) $95-111$

4. O.Cornea, Homotopical Dynamics: Suspension and Duality, Erg. Theory \& Dyn. Sys. 202 (2000) 379-391

5. O.Cornea, Homotopical Dynamics II: Hopf invariants, smoothings and the Morse complex, Preprint 1999

6. O.Cornea, Homotopical Dynamics III: Real singularities and hamiltonian flows, To appear Duke M. J.

7. O.Cornea, A.Ranicki, Morse cobordisms, Preprint 2001

8. G.Dula, On conic spaces, Springer LNM 1474 (1991) 38-58

9. Y.Eliashberg, M.Gromov, Lagrangian Intersection Theory - Finite dimensional appraoch, Geometry of Diff. Eq., AMS Transl. 86 (1998) 27-118

10. J.Franks, Morse-Smale flows and homotopy theory, Topology 18 (1979), 199-215

11. H.Hofer, Dynamics, Topology and Holomorphic Curves, Documenta Math. Extra Vol. ICM (1998) 255-280

12. H.Hofer, E.Zehnder, Symplectic Invariants and Hamiltonian Dynamics, Birkhäuser 1994

13. F.Latour, Existence de 1-formes fermeés non singulières dans une classe de cohomologie de de Rham, Pub. I.H.E.S. 80 (1994) 135-194

14. M.Mather, Pull-backs in Hmotopy Theory, Can.J.Math. 28 (1976), 225-263

15. J.Milnor, Morse Theory, Annals Math. Study 51 Princeton Press, 1963

16. Ch.McCord, Poincare-Lefschetz duality for the homology Conley index, Trans. of the A.M.S. 329 (1992) 233-252

17. J.Moser, Periodic orbits near an equilibrium and a theorem of A. Weinstein, Comm. in Pure and Appl. Math 29 (1976) 727-747

18. M.Mrozek, J.Reineck, M.Srzednicki, The Conley index over a base, Trans. A.M.S. 352 (2000) 4171-4194

19. S.Novikov, The Hamiltonian formalism and a multi-valued analogue of Morse thoery, Russian Math. Surv. 37 (1982) 1-56

20. R.Palais, Lusternik-Schnirelmann theory on Banach manifolds, Topology 5 (1966) 115-132

21. Ch.C.Pugh, C.Robinson, The $C^{1}$ closing Lemma, including Hamiltonians, Erg.Theory \& Dyn. Sys. 3 (1983) 261-313

22. P.H.Rabinowitz, Periodic Solutions of Hamiltonian Systems, Comm. Pure. Appl. Math. 31 (1978) 157-184

23. J.Reineck, Continuation to the minimal number of critical points in gradient flows, Duke Math. Journ. 68 (1992) 185-193

24. D.Salamon, Connected simple systems and the Conley index of isolated invariant sets, Trans. of the A.M.S. 291 (1985) 1-41

25. M.Schwarz, Morse homology, Birkhäuser 1993

26. L.Smith, Manifolds with few cells and the stable homotopy of spheres, Proc. AMS 311 (1972), 279-285

27. M.Spivak, Spaces satisfying Poincaré duality, Topology 6 (1967), 77-101

28. R.Switzer, Algebraic Topology - Homotopy and Homology, Springer 1975 
29. A.Weinstein, Periodic orbits for convex hamiltonian systems, Ann. of Math. 108 (1978) 507-518

30. G.Whitehead, Elements of Homotopy Theory, Springer 1978

Université de Lille 1, U.F.R. De Mathématiques \& U.M.R. 8524, 59655 VilLEneuve D'AscQ, France

E-mail address: cornea@gat.univ-lille1.fr

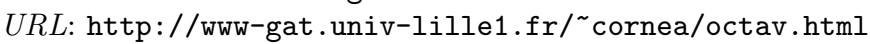

